\title{
Nonphotochemical hole-burning studies of energy transfer dynamics in antenna complexes of photosynthetic bacteria
}

\author{
by \\ Satoshi Matsuzaki \\ A thesis submitted to the graduate faculty \\ in partial fulfillment of the requirements for the degree of \\ MASTER OF SCIENCE
}

Major: Physical Chemistry

Major Professor: Gerald J. Small

Iowa State University

Ames, Iowa

2001 


\section{Graduate College \\ Iowa State University}

This is to certify that the Master's thesis of

\section{Satoshi Matsuzaki}

has met the thesis requirements of Iowa State University

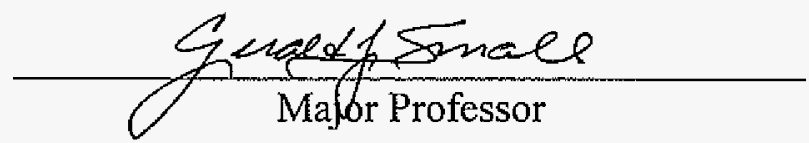

For the Major Program

For the Graduate College 


\section{TABLE OF CONTENTS}

CHAPTER 1. GENERAL INTRODUCTION TO BACTERIAL ANTENNA COMPLEXES

1.1 Thesis Organization

1.2 General Introduction to Photosynthesis 2

1.2.1 Historical Background 3

1.2.2 Chlorophyll and Bacteriochlorophyll 6

1.3 Light-harvesting Complexes (LHC) of Purple Bacteria 6

1.3.1 Structure of LH2 from Rps. acidophila (Strain 10050) 8

1.3.2 Excitonic Level Structure of the B850 Ring 14

$\begin{array}{lll}\text { 1.3.3 Excitation Energy Transfer Dynamics } & 17\end{array}$

1.4 Antenna Complex (FMO Complex) of Green Sulfer Bacteria 21

1.4.1 Structure of FMO Complex from Prosthecochloris aestuarii 22

1.4.2 Energy Transfer and Spectral Dynamics of the Three Lowest Energy States

CHAPTER 2. GENERAL INTRODUCTION TO NONPHOTOCHEMICAL HOLE-BURNING SPECTROSCOPY 35

2.1 General Introduction to Hole-burning Spectroscopy 35

2.2 Hole-burning Mechanism 41

2.3 Background Theories for Excitation Energy Transfer (EET) 47

2.3.1 Electron-Phonon Coupling 47

2.3.2 Single-site Absorption Profile and Absorption Spectrum $\quad 51$

2.3.3 Exciton Level Structures 54

CHAPTER 3. ENERGY TRANSFER KINETICS AND LOW ENERGY VIBRATIONAL STRUCTURE OF THE THREE LOWEST ENERGY QY-STATES OF THE FENNA-MATTHEWSOLSON (FMO) ANTENNA COMPLEX $\quad 64$

$\begin{array}{ll}\text { Abstract } & 64\end{array}$

$\begin{array}{ll}\text { Introduction } & 65\end{array}$

Materials and Methods $\quad 68$

$\begin{array}{ll}\text { Results and Discussion } & 70\end{array}$

Some general features of the hole spectra $\quad 70$

$\begin{array}{ll}\text { Low energy satelite hole structure } & 72\end{array}$

Energy transfer and spectral dynamics of the three lowest energy states $\quad 83$

Conclusions $\quad 85$

$\begin{array}{lr}\text { Acknowledgments } & 87\end{array}$

References $\quad 87$

Tables 90 
Figure Captions $\quad 93$

$\begin{array}{ll}\text { Figures } & 95\end{array}$

CHAPTER 4. ENERGY TRANSFER DYNAMICS IN LH2 COMPLEXES

OF RHODOPSEUDOMONAS ACIDOPHILA CONTAINING ONLY ONE B800 MOLECULE 104

$\begin{array}{ll}\text { Abstract } & 104\end{array}$

$\begin{array}{ll}\text { Introduction } & 105\end{array}$

$\begin{array}{ll}\text { Experimental } & 108\end{array}$

Results and Discussion 109

Assignment of the $799.7 \mathrm{~nm}$ band 109

B800 zero-phonon holewidths for B800-deficient LH2 111

Mechanistic aspects of B800-B800 transfer $\quad 112$

B850 excitonic structure in B800-deficient LH2 121

$\begin{array}{ll}\text { Conclusions and Final Remarks } & 123\end{array}$

Acknowledgments $\quad 125$

$\begin{array}{ll}\text { References } & 125\end{array}$

$\begin{array}{ll}\text { Figure Captions } & 129\end{array}$

$\begin{array}{ll}\text { Figures } & 131\end{array}$

CHAPTER 5. GENERAL CONCLUSIONS 136

$\begin{array}{ll}\text { APPENDIX. } & \text { 2-MODE/3-GAUSSIAN HOLE PROFILE SIMULATION } \\ & \text { PROGRAM }\end{array}$

$\begin{array}{ll}\text { ACKNOWLEDGMENTS } & 144\end{array}$ 


\section{CHAPTER 1. GENERAL INTRODUCTION TO BACTERIAL ANTENNA COMPLEXES}

\subsection{Thesis Organization}

This thesis contains the candidate's original work on excitonic structure and energy transfer dynamics of two bacterial antenna complexes as studied using spectral hole-burning spectroscopy. The general introduction is divided into two chapters (1. and 2.). Chapter 1 provides background material on photosynthesis and bacterial antenna complexes with emphasis on the two bacterial antenna systems related to the thesis research. Chapter 2 reviews the underlying principles and mechanism of persistent nonphotochemical holeburning (NPHB) spectroscopy. Relevant energy transfer theories are also discussed. Chapters 3 and 4 are papers by the candidate that have been published. Chapter 3 describes the application of NPHB spectroscopy to the Fenna-Matthews-Olson (FMO) complex from the green sulfur bacterium Prosthecochloris aestuarii; emphasis is on determination of the low energy vibrational structure that is important for understanding the energy transfer process associated within three lowest energy $Q_{y}$-states of the complex. The results are compared with those obtained earlier on the FMO complex from Chlorobium tepidum. In Chapter 4, the energy transfer dynamics of the B800 molecules of intact LH2 and B800deficient LH2 complexes of the purple bacterium Rhodopseudomonas acidophila are compared. New insights on the additional decay channel of the B800 ring of bacteriochlorophyll $a(\mathrm{BChl} a)$ molecules are provided. General conclusions are given in Chapter 5. A version of the hole spectrum simulation program written by the candidate for 
the FMO complex study (Chapter 3) is included as an appendix. The references for each chapter are given at the end of each chapter.

\subsection{General Introduction to Photosynthesis}

For over 200 years, research in the area of photosynthesis has been conducted to determine how photosynthetic organisms absorb solar photons and convert solar energy to chemical potential. An important motivation for this research has been to gain a fundamental understanding of the excitation energy transfer processes that occur within and between photosynthetic antenna complexes, the end result being transfer of solar energy to the reaction center complex where charge separation occurs. Chlorophyll (Chl) molecules are the most important pigments involved in light absorption and excitation energy transfer (EET). See Section 1.2.2 for $\mathrm{Chl}$ and bacteriochlorophyll (BChl) structures.

Fundamental studies of the light-driven processes of photosynthesis have proven indispensable in the design of artificial light harvesting and charge separation assemblies that can serve as key components in solar energy conversion technologies. The work presented in this thesis is concerned with the excitonic structure and EET dynamics of the BChl $a \mathrm{Qy}_{\mathrm{y}}\left(\mathrm{S}_{1}\right)$ states of the light harvesting 2 (LH2) complex of the purple bacterium Rps. acidophila (strain 10050) and the FMO antenna complex of the green sulfur bacterium $P$. aestuarii. For the LH2 complex, focus is on the EET channels of the B800 BChl $a$ molecules while for the FMO complex it is on the identification and characterization of the three lowest energy $\mathrm{Q}_{y^{-}}$ states and the kinetics and mechanism of EET between them and the determination of the nuclear spectral density that enters into the nonadiabatic rate expression. 


\subsubsection{Historical Background}

Photosynthesis is the physico-chemical process by which plants, algae and photosynthetic bacteria use light energy to drive the synthesis of organic compounds. In plants, algae and certain types of bacteria, the photosynthetic process results in the release of molecular oxygen and the removal from the atmosphere of carbon dioxide that is used to synthesize carbohydrates (oxygenic photosynthesis). Other types of bacteria use light energy to create organic compounds but do not produce oxygen (anoxygenic photosynthesis).

By the middle of the nineteenth century the key features of plant photosynthesis were known, namely, that plants could use light energy to make carbohydrates from $\mathrm{CO}_{2}$ and water. The empirical equation representing the net reaction of photosynthesis for oxygen evolving organisms is:

$$
\mathrm{CO}_{2}+2 \mathrm{H}_{2} \mathrm{O}+h v \rightarrow\left[\mathrm{CH}_{2} \mathrm{O}\right]+\mathrm{O}_{2}+\mathrm{H}_{2} \mathrm{O},
$$

where $\left[\mathrm{CH}_{2} \mathrm{O}\right]$ represents a carbohydrate (e.g., glucose). The synthesis of carbohydrate from carbon and water requires a large input of light energy. The standard free energy for the reduction of one mole of $\mathrm{CO}_{2}$ to the level of glucose is $+478 \mathrm{~kJ} / \mathrm{mol}$. Because glucose is often an intermediate product of photosynthesis, the net equation of photosynthesis is frequently written as:

$$
6 \mathrm{CO}_{2}+12 \mathrm{H}_{2} \mathrm{O}+{ }_{h v} \rightarrow \mathrm{C}_{6} \mathrm{H}_{12} \mathrm{O}_{6}+6 \mathrm{O}_{2}+6 \mathrm{H}_{2} \mathrm{O},
$$

The standard free energy for the synthesis of glucose is $+2870 \mathrm{~kJ} / \mathrm{mol}[1]$.

Early scientists studying photosynthesis concluded that the $\mathrm{O}_{2}$ released by plants came from $\mathrm{CO}_{2}$, which was thought to be split by light energy. In the 1930s, comparison of bacterial and plant photosynthesis lead van Niel to propose the general equation of photosynthesis that applies to plants, algae and photosynthetic bacteria [1]. Van Niel was 
aware that some photosynthetic bacteria could use hydrogen sulfide $\left(\mathrm{H}_{2} \mathrm{~S}\right)$ instead of water for photosynthesis and that these organisms released sulfur instead of oxygen. Van Niel, among others, concluded that photosynthesis depends on electron donation and acceptor reactions and that the $\mathrm{O}_{2}$ released during photosynthesis comes from the oxidation of water. Van Niel's generalized equation is:

$$
\mathrm{CO}_{2}+2 \mathrm{H}_{2} \mathrm{~A}+h v \rightarrow\left[\mathrm{CH}_{2} \mathrm{O}\right]+2 \mathrm{~A}+\mathrm{H}_{2} \mathrm{O} .
$$

In oxygenic photosynthesis, $2 \mathrm{~A}$ is $\mathrm{O}_{2}$, whereas in anoxygenic photosynthesis, which occurs in some photosynthetic bacteria, the electron donor can be an inorganic hydrogen donor, such as $\mathrm{H}_{2} \mathrm{~S}$, or an organic hydrogen donor, such as succinate. Experimental evidence that molecular oxygen came from water was provided by Hill and Scarisbrick [2] who demonstrated oxygen evolution in the absence of $\mathrm{CO}_{2}$ in illuminated chloroplasts and by Ruben et al. who used ${ }^{18} \mathrm{O}$ enriched water [3].

The biochemical conversion of $\mathrm{CO}_{2}$ and $\mathrm{H}_{2} \mathrm{O}$ to carbohydrate is a reduction reaction that involves the rearrangement of covalent bonds between carbon, hydrogen and oxygen. The energy for the reduction of carbon is provided by energy-rich molecules that are produced by the light driven electron transfer reactions. Carbon reduction can occur in the dark and involves a series of biochemical reactions that were elucidated by Calvin, Benson and Bassham in the late 1940s and early 1950s.

In 1954, Arnon and coworkers discovered that plants use light energy to produce ATP, an organic molecule that serves as an energy source for many biochemical reactions. Also in 1954, Frenkel discovered photosynthetic bacteria are capable of producing ATP, driven by light energy [4]. During the same period, Duysens was able to show that the primary photochemical reaction of photosynthesis is an oxidation/reduction reaction that 
occurs in a protein complex (the reaction center). Over the next few years the work of several groups, including those of Emerson, Kok, Duysens, Hill and Witt, combined to prove that plants, algae and cyanobacteria require two reaction centers, photosystem II and photosystem I, operating in series [5,6]. In 1961, Mitchell suggested that cells can store energy by creating an electric field or a proton gradient across a membrane [7]. Mitchell's proposal that energy is stored as an electrochemical gradient across a vesicular membrane opened the door for understanding energy transformation by membrane systems.

Most of the proteins required for the conversion of light energy and electron transfer reactions of photosynthesis are located in membranes. Despite decades of work, efforts to determine the structure of membrane bound proteins had little success. This changed in the 1980s when Deisenhofer, Michel, Huber and co-workers determined the structure of the reaction center of the purple bacterium Rhodospeudomonas viridis by X-ray diffraction [810].

It is important to emphasize that knowledge of the structure of a photosynthetic antenna complex is essential for arriving at a firm understanding of the quantum mechanics of EET since, for example, the orientations of and distances between the Chl molecules bound to the protein are required for calculation of excitonic Chl-Chl coupling energies. It is to be appreciated, however, that a structure alone is insufficient for achieving a firm understanding of EET. For instance, the excitation energies of different Chl molecules in a complex are generally not identical, either because different $\mathrm{Chl}$ molecules are symmetry inequivalent and/or because of the glass-like structural heterogeneity of proteins. Furthermore, calculation of EET rates requires knowledge of the nuclear spectral density associated with energy conservation. This density involves intramolecular $\mathrm{Chl}$ vibrations and 
protein phonons. Clearly, data from a variety of frequency and time domain experiments and theoretical simulations are essential to the study of EET.

\subsubsection{Chlorophylls and Bacteriochlorophylls}

Plant photosynthesis is driven primarily by visible light that is absorbed by pigment molecules (mainly chlorophylls (Chl) or bacteriochlorophylls (BChl) and carotenoids). Chl $a$ and Chl $b$ are found in the antenna complexes of photosystem I and II of highter plants while $\mathrm{BChl} a$ and BChl $b$ exist in antenna complexes of bacteria. The chemical structures of the $\mathrm{Chl} a$ and BChl $a$ molecules are shown in Figure 1.1(a) and (b) (in Chl $b$ and BChl $b$ the $\mathrm{CH}_{3}$ group in ring II is replaced by a CHO group). Absorption spectra of BChl $a$ are shown in Figure 1.1(c). Monomeric BChl $a$ in organic solvents exhibits a $\mathrm{Q}_{\mathrm{y}}$-absorption band around $777 \mathrm{~nm}$. Typically, 200-300 pigment molecules are bound to light-harvesting protein complexes, which serve as antennae, located in the photosynthetic membrane to collect light.

The following two sections (Section 1.3 and 1.4) describe the structures and the arrangements of two aforementioned specific bacterial antenna complexes, which lead research interests of excitonic structure and EET dynamics of those antenna complexes (Chapter 3 and 4).

\subsection{Light-harvesting Complexes (LHC) of Purple Bacteria}

The LHC or antenna complex of purple bacteria is located in the intracytoplasmic membrane in the form of vesicular, lamellar and rod shaped structures. The fundamental structural and functional element of antenna complexes is a heterodimer of $\alpha$ - and $\beta$ polypeptides (apoproteins) [12]. The binding sites for BChl molecules (via the central 
(a)

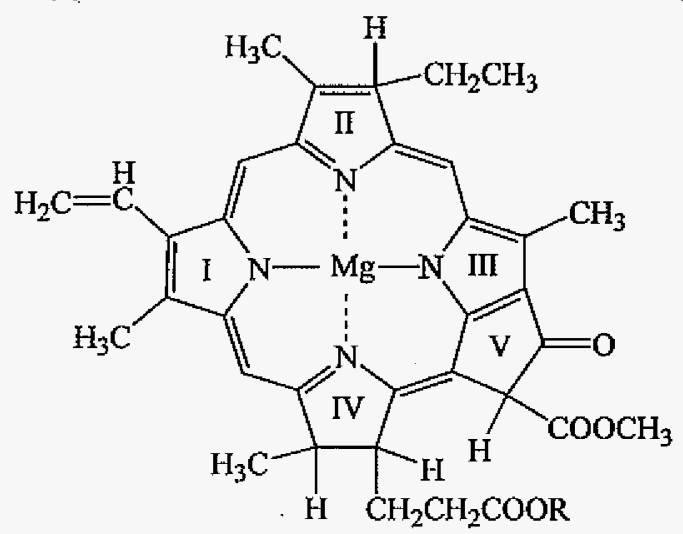

(b)

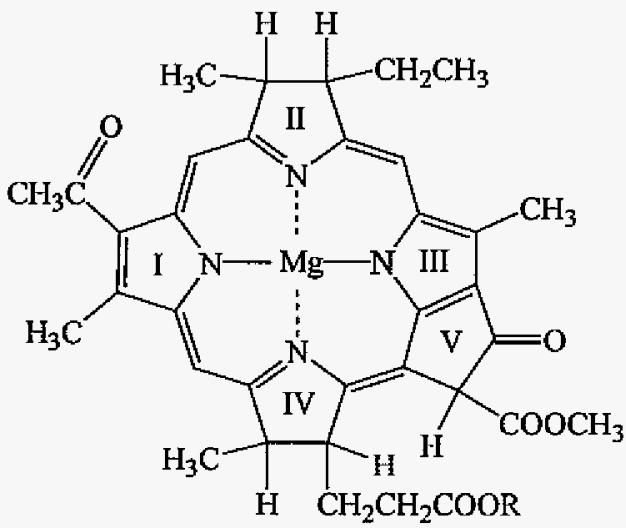<smiles>[R][CH]C=C(C)CCCC(C)CCCC(C)CCCC(C)C</smiles>

(c)

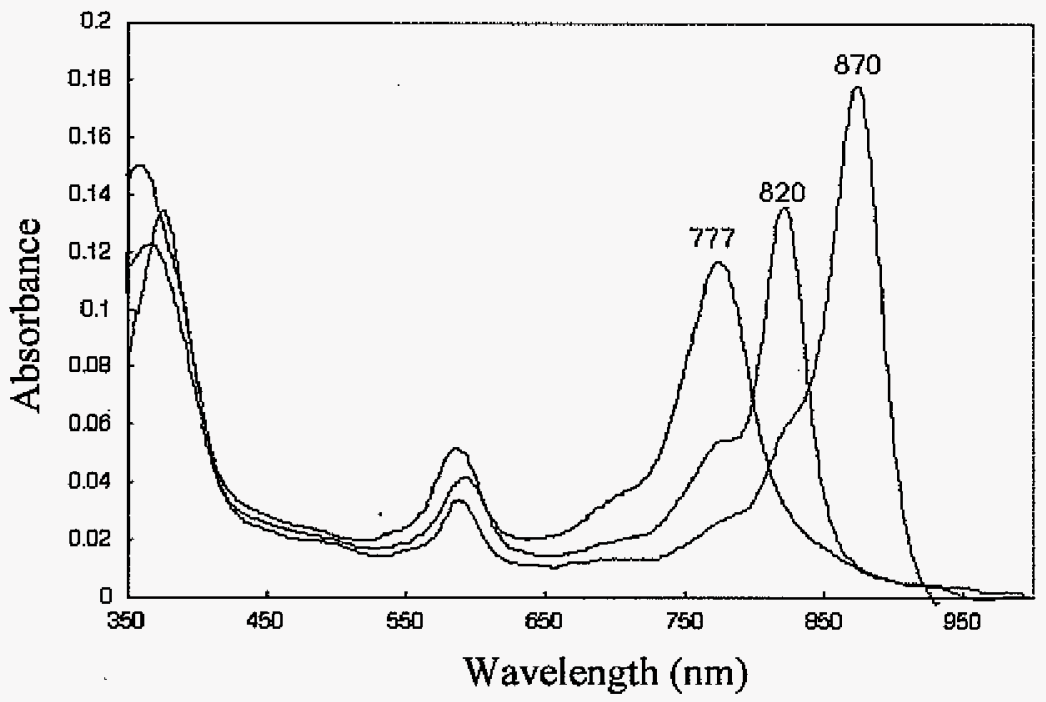

Figure 1.1 Structures of (a) a chlorophyll $a$ and (b) a bacteriochlorophyll $a$. The phytyl chain is abbreviated as $\mathrm{R}$ in both of the structures. The structures are distinguished, in part, by the saturation/unsaturation in ring II. Part (c) shows room temperature absorption spectra of $\mathrm{BChl} a$. The blue spectrum is that of $\mathrm{BChl} a$ in an organic solvent. The pink and red spectra are those of a dimer and the native $\mathrm{LH} 1$ complex that is a cyclic array of $16 \mathrm{BChl} a$ dimers, respectively [11]. 
magnesium atom) are the conserved histidine residues found in all $\alpha$ - and $\beta$-chains in the hydrophobic domain, which is the central part of the $\alpha$ - and $\beta$-polypeptides in $\alpha$-helix form $[12,13]$.

The components of the antenna complexes in purple bacteria depend on the individual types, their heterogeneous energy transfer systems and strains. Rhodospirillum rubrum and Rhodopseudomonas viridis have only one complex, $\mathrm{B} 870$ and $\mathrm{B} 1020$, respectively, where, e.g., $\mathrm{B} 870$ indicates $\mathrm{BChl}$ molecules that absorb at $870 \mathrm{~nm}$ at room temperature.

Rhodopseudomonas sphaeroides has two complexes (B870, B800-850) and Chromatium vinosum has three complexes (B870, B800-850, B800-820) [12]. In the case of

Rhodopseudomonas (Rps.) acidophila (strain 10050), there exist B875 and B800-850 complexes. The $32 \mathrm{BChl} a$ molecules of the $\mathrm{B} 875$ complex (core antenna) are arranged in a circle with $\mathrm{C}_{16}$ symmetry that surrounds the reaction center (RC) [14]. The $\mathrm{B} 875$ complex is commonly referred to as the light-harvesting 1 (LH1) complex. On the other hand, the B800850 complex (peripheral antennae) is referred to as the LH2 complex and is located peripherally to the RC-LH1 core. The concentric circular arrangement of the LH1 and LH2 complexes in a modeled bacterial photosynthetic unit (PSU) is shown in Figure 1.2.

\subsubsection{Structure of LH2 from Rps. acidophila (Strain 10050)}

Most recently, the crystal structure of the LH2 of Rps. acidophila (strain 10050) has been determined at $2.5 \AA$ resolution by X-ray diffraction [17]. The $\alpha$-apoprotein contains 53 amino acids and the $\beta$ apoprotein has 41 amino acids. The LH2 complex is a ring of nine identical units, each unit containing $\alpha$ - and $\beta$-polypeptides, three $\mathrm{BChl} a$ molecules and at least one carotenoid. The pair of $\alpha$ - and $\beta$ - polypeptides, which constitute a minimum 


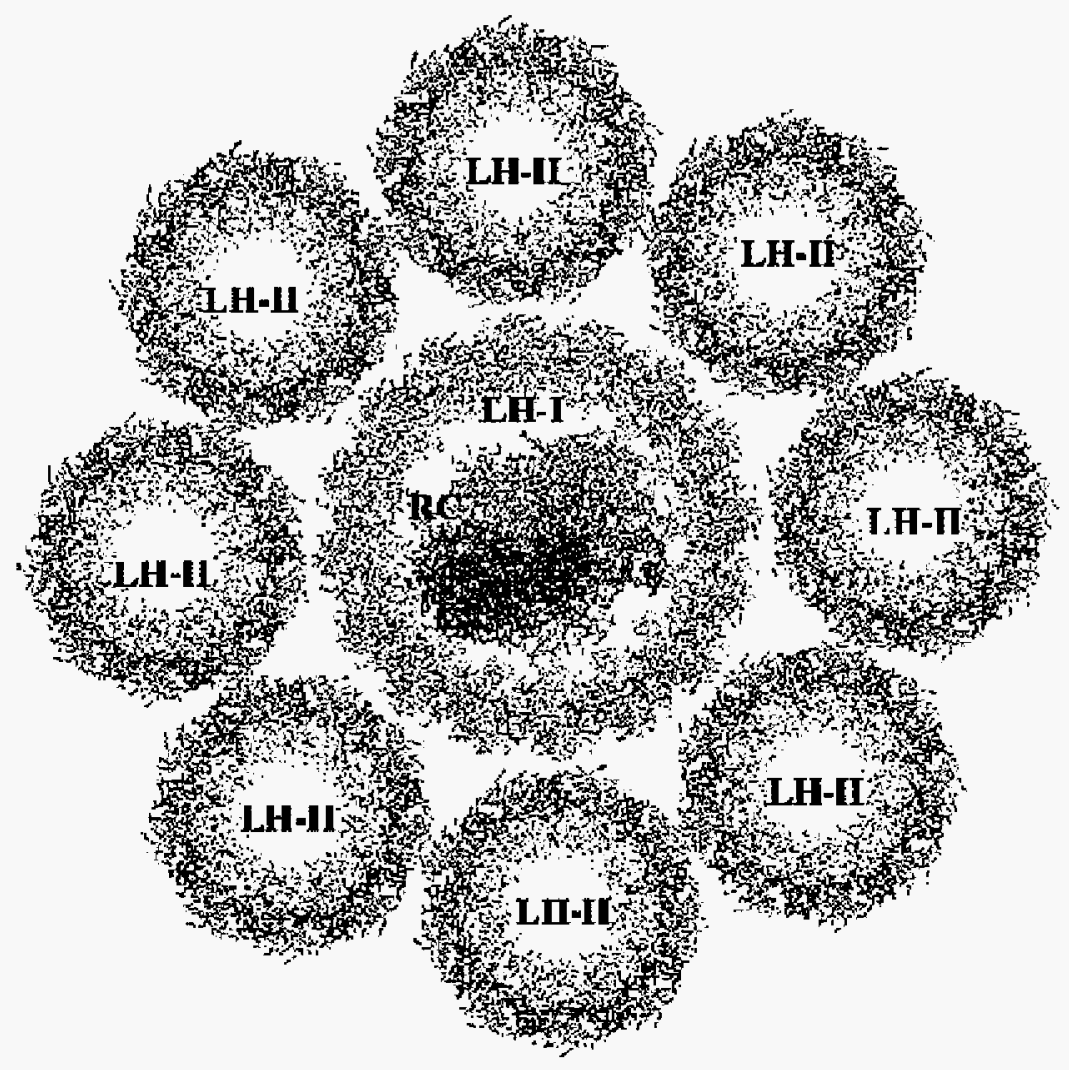

Figure 1.2 Arrangement of light-harvesting complexes in the modeled bacterial photosynthetic unit (PSU) of $R b$. sphaeroides, in the plane of the special pair of the RC. Original picture was produced with the program VMD [15] and adapted from [16]. 
repeating unit that then aggregates to form the fully functional in vivo antenna, is called the protomer. The crystal structure shows that the active assembly consists of two concentric cylinders of helical protein subunits that enclose the pigment molecules. The transmembrane helices of nine $\alpha$ apoproteins are packed side by side to form a hollow cylinder and the nine helical $\beta$ apoproteins are arranged radially with the $\alpha$ apoproteins forming an outer cylinder. See Figure 1.3(b). The $\alpha$ apoprotein helices are almost parallel to the nine-fold rotation axis to within $2^{\circ}$, whereas the $\beta$ helices are inclined by $15^{\circ}$ relative to this axis.

Within the LH2 complex, there are two rings of BChl a molecules, one set of 18 molecules close to the membrane surface (B850) and another set of 9 molecules in the middle of the bilayer (B800). The B850 pigment molecules are positioned vertically with respect to the membrane plane, whereas the $\mathrm{B} 800$ pigments lie almost parallel to the membrane plane. The BChl $\alpha$-binding histidines of the $\alpha$ - (His31) and $\beta$ - (His30) apoproteins face outwards and inwards, respectively, forming a complete ring of 18 overlapping $\mathrm{BChl}$ molecules (see Figures 1.3 and 1.4). The planes of the $18 \mathrm{~B} 850 \mathrm{BChls}$ are perpendicular to the membrane surface and the phytyl chains descend into the hydrophobic core of the assembly. Within a protomer, the $\mathrm{Mg}-\mathrm{Mg}$ distance is $8.9 \AA$, and between adjacent protomers, the shortest distance is $9.6 \AA$. There are van der Waals contacts at the periphery of the bacteriochlorin systems. The conformations of the phytyl chains of the $\alpha$ and $\beta$ B850 BChls differ (Figure 1.5). In the $\alpha$ form, the chain is slightly more extended, whereas in the $\beta$ form, the chain is bent and passes parallel to the $B 800$ ring at a distance of $\sim 4.0 \AA$. The nine $\mathrm{B} 800 \mathrm{BChl} a$ molecules are located between the $\beta$ apoproteins, with the planes of bacteriochlorins almost parallel to the presumed cytoplasmic membrane surface and about 11 $\AA$ away from it. The distance between the central Mg atoms of the $\mathrm{B} 800$ molecules is $21.2 \AA$ 
(a)

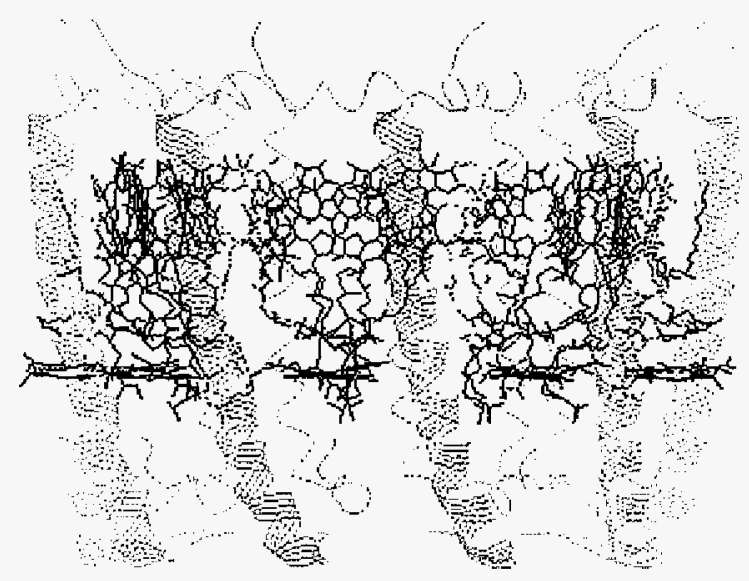

(b)

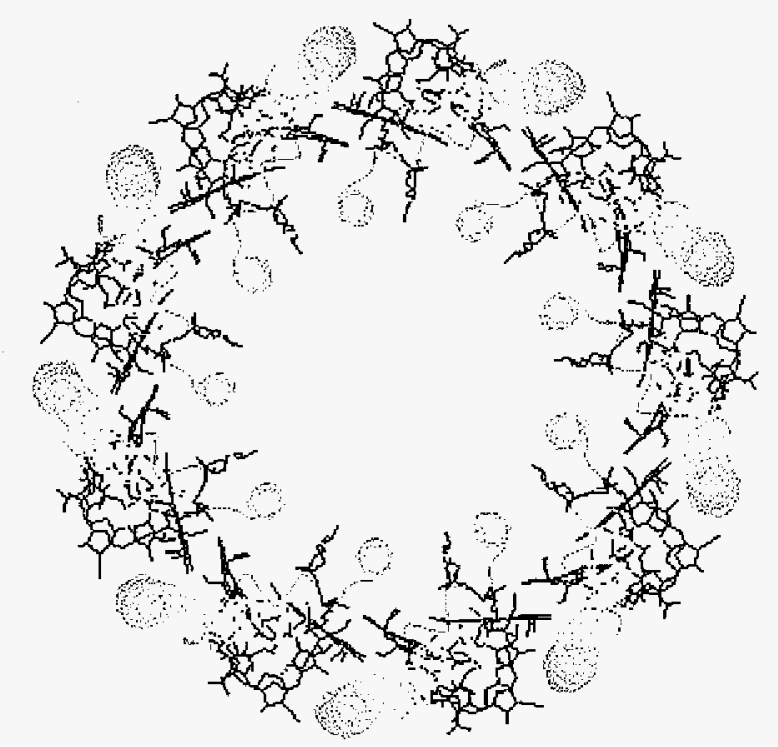

Figure 1.3 Part (a) and (b) show cyclically arranged $\alpha-$, $\beta$-polypeptides of LH2 complex from Rps. acidophila (strain 10050) [17,18]. $\alpha$-polypeptides are located on the inner circle with $\sim 18 \AA \AA$ radius, where $\beta$-polypeptides are the outer shell having $\sim 34 \AA$ radius. BChls are expressed in wireframe forms, where as the rest of the proteins are in strand forms. The figures were generated using the crystallographic coordinates with the program RasMol [15]. 


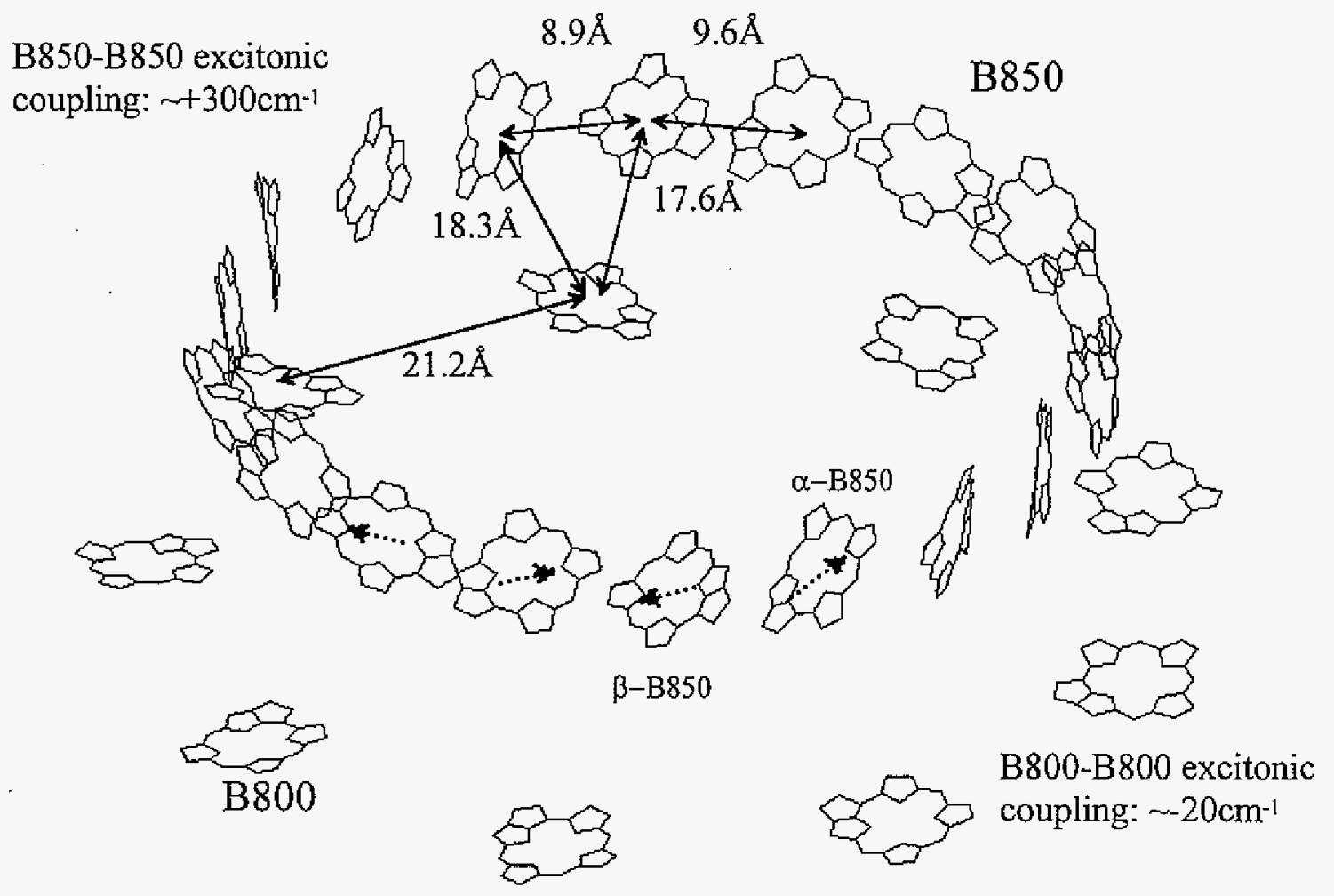

Figure 1.4 Arrangement of the $27 \mathrm{BChls}$ in the LH2 complex from Rps. acidophila (strain 10050). All the side chains on the BChls are omitted for clarity. As shown, the upper ring contains 18 B850 molecules, while the lower ring contains nine $\mathrm{B} 800$ molecules. Each of the $\mathrm{Mg}-\mathrm{Mg}$ distances between the $\mathrm{BChls}$ is presented with solid arrows. Dotted arrows are also drawn to show the directions of $\mathrm{Q}_{\mathrm{y}}$-transition dipole of the BChls. Excitonic coupling energies between neighboring B800-B800 molecules and between B850B850 molecules $[19,20]$ are also shown in the figure. The figure was modified from the one created by the RasMol program [15]. 

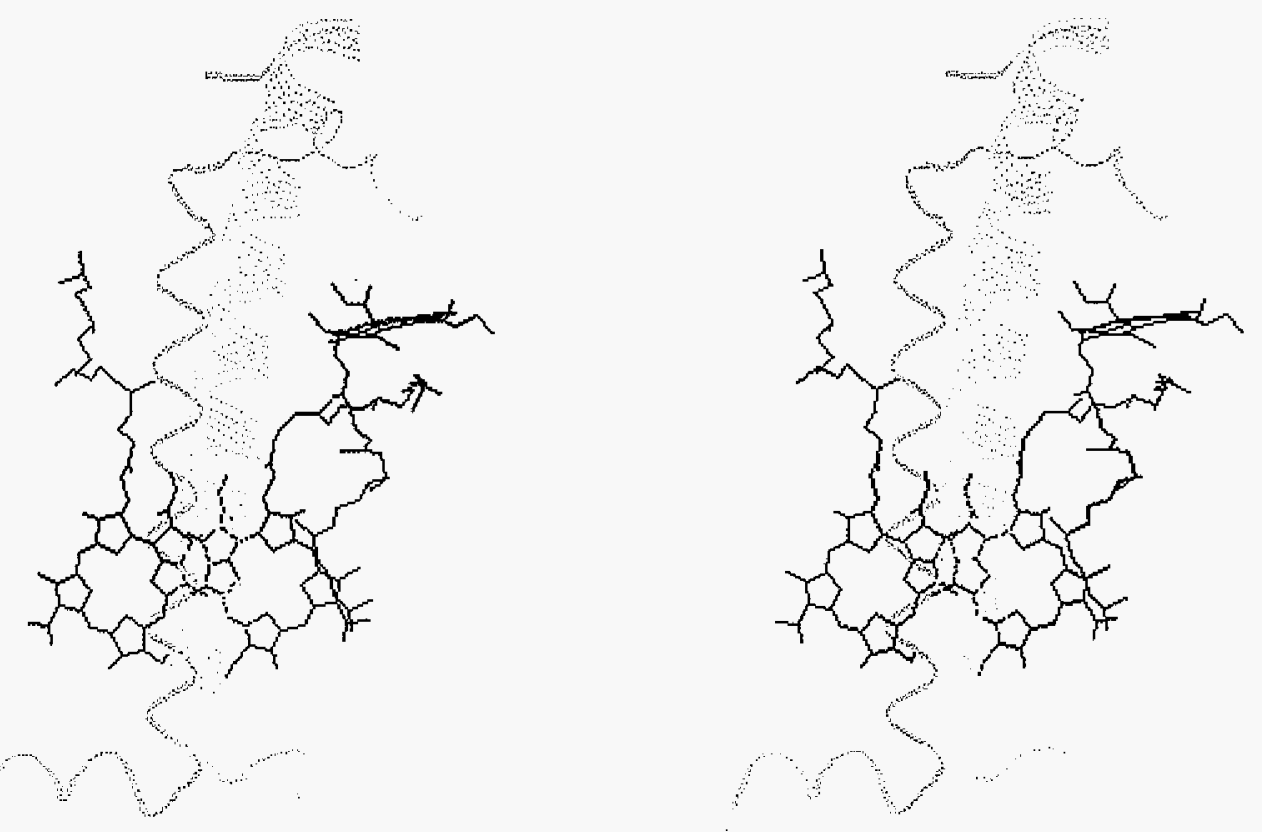

Figure 1.5 Stereographical view of the protomer of the LH2 complex from Rps. acidophila (strain 10050), which is the repeating unit of the 9-fold symmetry complex. The front tube represents the $\alpha$-apoprotein, where as the back ribbon is the $\beta$-apoprotein. This figure is a view from the $\mathrm{C}_{9}$ cylinder center. The $\mathrm{BChl} a$ molecule that is perpendicular to the $\mathrm{C}_{9}$ axis is the $\mathrm{B} 800$ molecule. Two strongly coupled B850 molecules are located between the $\alpha$ and $\beta$-polypeptides. The $\alpha-\mathrm{B} 850$ molecule is in the right (red) and the $\beta-$ B850 molecule is in the left (blue). The figure was created with RasMol [15]. 
and the distance between the closest pair of $\mathrm{B} 800$ and $\mathrm{B} 850$ molecules is $17.6 \AA$. Excitonic coupling between the nearest neighbor B800 molecules was determined to be $\sim-20 \mathrm{~cm}^{-1}$, whereas the corresponding coupling between the B850 molecules on adjacent subunits is $\sim$ $+300 \mathrm{~cm}^{-1}[19,20]$, see Figure 1.4. Also, there are at least nine membrane-spanning carotenoids (rhodopin-glucoside), each of which span the membrane and makes close contact (less than $3.5 \AA$ ) with both the B850 and B800 BChls. Recently, an electron density map showed that the $\mathrm{LH} 1$ complex contains $32 \mathrm{~B} 875 \mathrm{BChls}$ and has a 16 -fold symmetric ring of $\alpha \beta$-heterodimers, with the RC in its center [21].

\subsubsection{Excitonic Level Structure of the B850 Ring}

Hamiltonian models for exciton levels of $\mathrm{C}_{n}$ cyclic ringed choromophores (dimers for the case at hand), which were utilized for simulations in Chapter 4, were developed in Refs. [22-24]. The Hamiltonian in the absence of disorder is given [23] by

$$
H_{0}=e \sum_{\alpha=0}^{n-1}|\alpha\rangle\left\langle\alpha\left|+\sum_{\alpha, \beta=0}^{n-1} V_{\alpha, \beta}\right| \alpha\right\rangle\langle\beta|,
$$

where $\alpha$ and $\beta$ denote chromophores or sites and $e$ is the excitation energy of the chromophore. The eigenfunctions of $H_{0}$ are delocalized and determined by symmetry to be [25]

$$
|j\rangle=n^{-1 / 2} \sum_{\alpha}^{n-1} B^{j \alpha^{*}}|\alpha\rangle,
$$

where $B=\exp (\mathrm{i} 2 \pi / n)$ and $j=0,1, \ldots, n^{-1}$ with ${ }_{n}$ the number of dimers in the ring. Then, with the nearest dimer-dimer coupling approximation, the exciton energies are given by [23]

$$
E^{j}=e+2 V \cos (2 \pi j / n)
$$


with the nearest neighbor dimer-dimer coupling $V$. It can be rewritten for the energies of two exciton manifolds (lower- and upper-level of the dimer) as

$$
E_{l}^{j}=e_{l}+2 V_{l} \cos (2 \pi j / n)
$$

and

$$
E_{t}^{j}=e_{n}+2 V_{u} \cos (2 \pi j / n)
$$

where $e_{l}$ and $e_{u}$ correspond to the energies of the two levels and $V_{l}$ and $V_{u}$ are the nearest neighbor dimer-dimer coupling energies for the lower and upper manifolds, respectively. The coupling between the exciton levels of different manifolds is given by

$$
H_{u l}^{j}=2 V_{u l} \cos (2 \pi j / n)
$$

The dimer-dimer couplings $V_{l}, V_{u}$ and $V_{u l}$ can be determined using the monomer-monomer coupling energies [24]. Sample exciton level simulation results for the B850 ring (in the absence of energy disorder) are shown in Figure 1.6. The coupling parameters for the simulation are given in the figure caption. For $n=9$, from the 1-dimensional irreducible representations of the $\mathrm{C}_{9}$ group, there exist one non-degenerate level $(\mathrm{A})$ and 4 degenerate levels $\left(\mathrm{E}_{1}, \mathrm{E}_{2}, \mathrm{E}_{3}, \mathrm{E}_{4}\right)$, each corresponding to $j=0,\{1,8\},\{2,7\},\{3,6\}$ and $\{4,5\}$, respectively. The lowest $E_{1}$ level carries most of the absorption intensity [19] and because of this the $E_{1}$ level is placed at the maximum of the $B 850$ band. It should be noted that the $A$ levels and upper $E_{I}$ level, even though the levels are symmetry allowed in absorption, are highly forbidden by the structural arrangement of the $Q_{y}$-transition dipoles. Based on simulations by Sauer et al., the lowest A level is expected to carry less than $1 \%$ of the absorption intensity of the $\mathrm{B} 850$ band in the absence of energy disorder [19]. For experimental support, the zero-phonon hole action spectra reported in Ref. [26] located the 


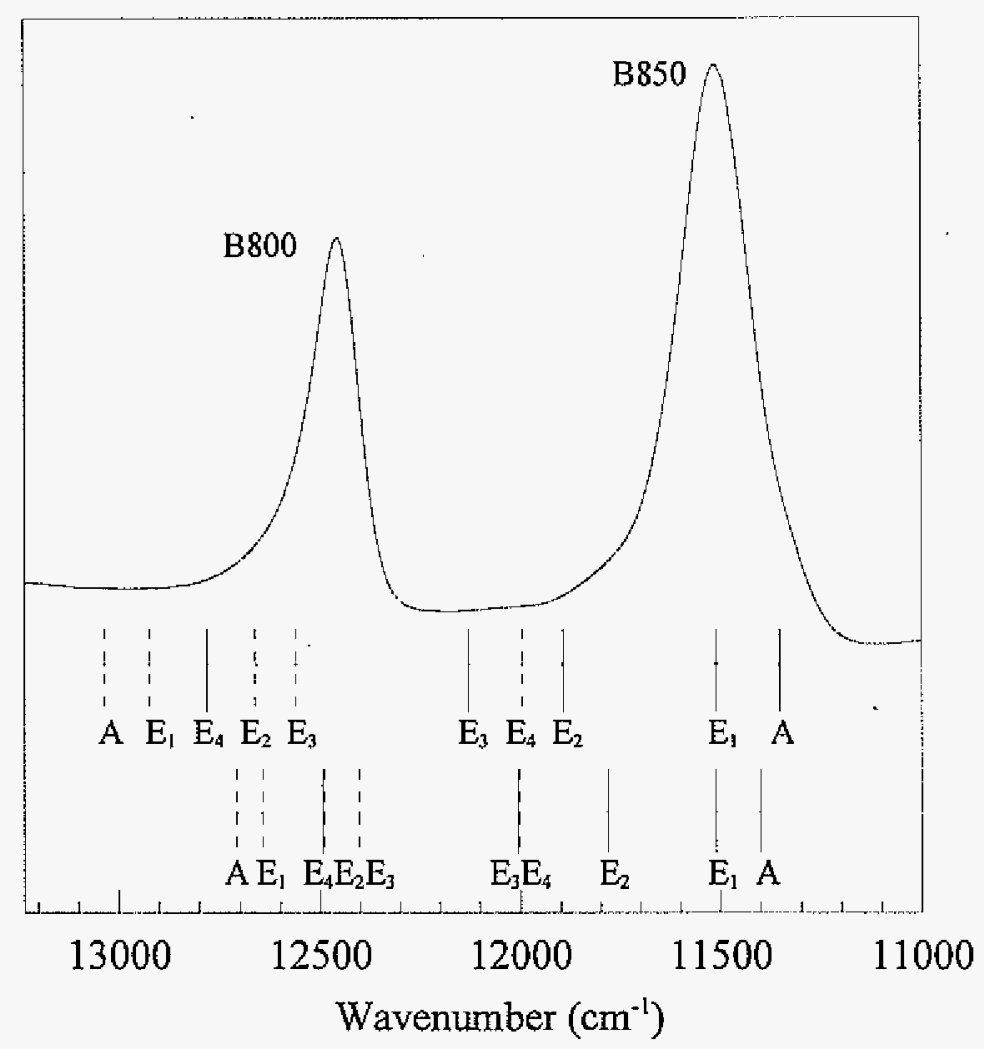

Figure 1.6 Exciton levels of the B850 ring based on simulation with the Hamiltonian defined by Eq. (4). The simulation parameter sets for $e_{u}-e_{l}, V_{l}, V_{u}$ and $V_{u l}$ are $600,-270,170$ and 200 (for upper set - the one presented in Chapter 4) and $600,-200,100$ and 130 (for lower set - given in Sauer et al. [19]), respectively, in the unit of $\mathrm{cm}^{-1}$. The solid and dashed vertical lines represent exciton levels associated with the lower- and upper-manifolds, respectively. The labels $A, E_{1}, E_{2}, E_{3}$ and $E_{4}$ correspond to the irreducible representations of the $\mathrm{C}_{9}$ group. See text. 
weakly absorbing A level about $200 \mathrm{~cm}^{-1}$ lower in energy than the strongly allowed E1 level. Also, Stark hole burning data led to a very small dipole moment change for the A level, as predicted by theory in the absence of energy disorder due to the intrinsic structural heterogeneity of proteins [27].

\subsubsection{Excitation Energy Transfer Dynamics}

The direction of energy transfer within the bacterial photosynthetic unit is not random, but is guided by the energy gradient going from B800 to B850 to B875 (LH1) and then to the RC (see Figure 1.7). The large separation $(\sim 21 \AA)$ between the B800 BChls and concomitant weak nearest neighbor electronic coupling $\left(\sim-20 \mathrm{~cm}^{-1}\right)$ as well as energy disorder due to the intrinsic structural heterogeneity of proteins indicate that the $Q_{y}$ excitation of the $\mathrm{B} 800$ ring can be viewed as highly localized on individual BChl molecules. The results of recent spectroscopic studies of single LH2 complexes are consistent with this [29,30]. In contrast, the B850 BChls are sufficiently closely spaced $(\sim 9 \AA)$ that it is necessary to consider them as strongly excitonically coupled aggregates [19]. That is, the $S_{1}\left(Q_{y}\right)$ wavefunctions are significantly delocalized even when energy disorder due to protein structural heterogeneity is taken into account (see Ref. [29] and references therein). The sufficiently close distance between B800 and B850 allows B800 $\rightarrow$ B850 energy transfer with $\sim 700$ fs time constant at room temperature [31]. The time constant at $4 \mathrm{~K}$ is only slightly longer, $\sim 2$ ps [32]. The $Q_{y}$ transition dipole moments of neighboring B800 and $\mathrm{B} 850 \mathrm{BChls}$ are nearly parallel to each other, which is to say that they are optimally aligned for a Förster dipole-dipole energy transfer mechanism (modified for the excitonic level structure of the $\mathrm{B} 850$ ring). Once the excitation reaches a $\mathrm{B} 850$ molecule, the strong 


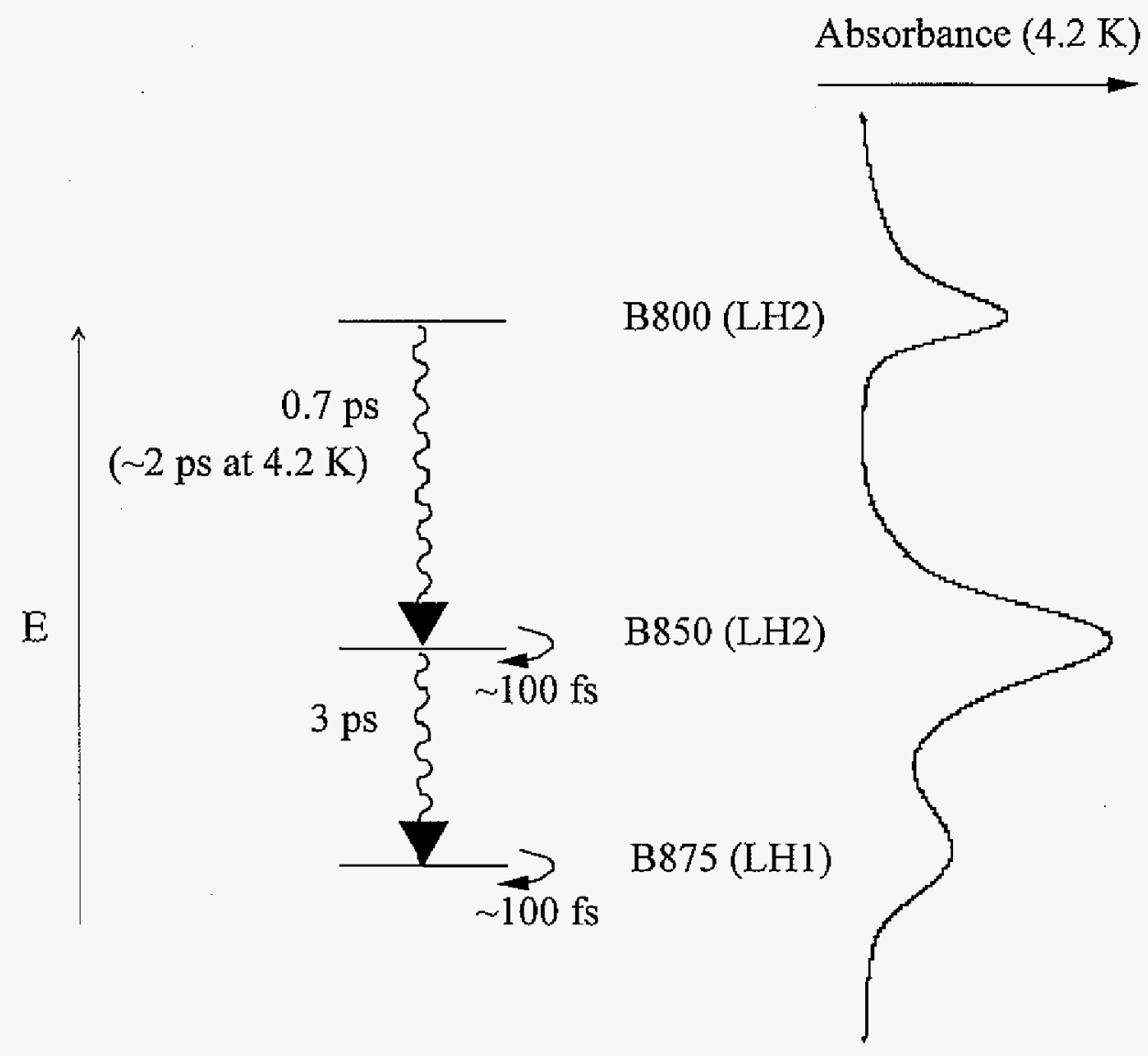

Figure 1.7 Low temperature ( $4.2 \mathrm{~K}$ ) absorption spectrum of chromatophores from $R p s$. acidophila (strain 10050). At $4.2 \mathrm{~K}$, the B800, B850 and B875 bands are located at $\sim 804 \mathrm{~nm}, 870 \mathrm{~nm}$ and $905 \mathrm{~nm}$, respectively. The B850 and B875 bands positions depend strongly on temperature [26], while the location of B800 band is almost temperature independent. The time constants of 0.7 and 3 ps apply respectively to the $\mathrm{B} 800 \rightarrow \mathrm{B} 850$ and $\mathrm{B} 850 \rightarrow \mathrm{B} 875$ energy transfer times at room temperature. The $\sim 100 \mathrm{fs}$ time constants correspond to inter-exciton level relaxations within the $\mathrm{B} 850$ and $\mathrm{B} 875$ rings (for a review see Ref. [28]). This figure was adapted from Ref. [46]. 
excitonic couplings between $\mathrm{B} 850$ molecules ensure that this excitation visits the other $\mathrm{B} 850$ BChls in about $100-200 \mathrm{fs}[33,34]$. The B850 excitation is available for transfer from any part of the ring to the LH1 complex. When the LH2 and LH1 (B875) rings are in close proximity, the energy can transfer to an adjacent LH1 complex, which takes place in $\sim 3$ ps at room temperature [35], Figure 1.7. Moreover, recent room temperature pump-probe experiment on LH1 found a time constant of $\sim 100$ fs for intra-LH1 energy transfer [36]. Readers who are interested in interactions and energy transfer between carotenoid and $\mathrm{BChl}$ molecules of LH2 should refer to the recent review by Sundström et al. [28].

Recent time-resolved experiments on variety of LH2 systems that followed the original work of Kramer et al. [37] in 1984 confirmed the presence of fast relaxation processes within the $\mathrm{B} 800$ band. Hess et al. reported a 300 fs intra-B800 relaxation channel based on isotropic and anisotropic decays at $77 \mathrm{~K}$ for LH2 of $R b$. sphaeroides [38]. A similar $400 \mathrm{fs}$ intraband relaxation process was observed for $\mathrm{B} 800$ of Rps. acidophila at $19 \mathrm{~K}$ [39] and $\sim 1$ ps anisotropy decay in the B800 band of the same bacterium at room temperature was also reported [40]. Since the energy transfer within the B800 ring is believed to be explicable in terms of the Förster equation for energy transfer (in the weak coupling limit), the $\mathrm{B} 800-\mathrm{B} 800$ transfer rate is predicted to be temperature dependent. As an alternative interpretation, however, Wu et al. proposed an additional decay channel of B800 (at low temperature) for the $400 \mathrm{fs}$ fast relaxation process, based on the result that zerophonon holes ( $\mathrm{ZPH})$ near the $\mathrm{B} 800$ absorption maximum and at lower energy within the B800 band exhibited almost constant holewidths, which implies that the widths are associated with $\mathrm{B} 800 \rightarrow \mathrm{B} 850$ energy transfer, while the widths of ZPH burned on the high energy side of B800 increase as the burn frequency increases [39]. It should be noted that 
this "additional" channel was also supported by low temperature pump-probe experiments [28] and recent spectroscopic studies of single complexes of Rps. acidophila [30]. As discussed in Chapter 4, there were several explanations considered for this channel.

$\mathrm{B} 800$ to $\mathrm{B} 850$ energy transfer processes in Rb. sphaeroides and Rps. acidophila have also been studied in detail. In both species, very similar transfer rates $(\sim 0.7 \mathrm{ps}$ at room temperature) were reported $[40,41]$. In the low temperature limit, Wu et al. [39] reported the transfer times of $1.6 \pm 0.2 \mathrm{ps}$ and $1.9 \pm 0.2 \mathrm{ps}$ determined by femtosecond pump-probe and NPHB spectroscopies, respectively. Recently, quantitative Förster-type calculations of this energy transfer process were performed $[20,39,42,43]$ using reasonable values for the Franck-Condon (FC) factors of the vibrational modes of $\mathrm{BChl} a$. The calculations yielded a considerably longer B800 $\rightarrow$ B850 transfer time than the measured values, which suggested that there may be an additional channel for $\mathrm{B} 800 \rightarrow \mathrm{B} 850$ relaxation. Hole-burning experiments at high pressure [39] suggested that this additional channel may be due to the interaction of excited B800 molecules with quasi-degenerate upper exciton levels of B850.

Based on the theory of Ref. [44], the following weak coupling non-adiabatic rate expression with consideration of energy disorder effect was employed for the energy transfer kinetic studies in Refs. [39,45]:

$$
\begin{gathered}
\left\langle k_{D A}(T)\right\rangle=2 \pi n^{-4} V^{2} e^{-\sum_{i} s_{t}}\left(1-e^{-\hat{s}}\right) \times \\
\sum_{i} 2 \mathrm{FC}_{i}\left[\frac{\exp \left\{-\left(\Omega_{0}-\omega_{i}-S \omega_{m}\right)^{2} / 2\left(\Gamma^{2}+Z^{2}\right)\right\}}{\sqrt{2 \pi\left(\Gamma^{2}+Z^{2}\right)}}\right],
\end{gathered}
$$

where $\Omega_{0}$ is the average value of the donor-acceptor energy gap, $\Gamma^{2}$ is the variance of the gap, $V$ is the electronic coupling and $n$ is the refractive index factor. $Z^{2}$ is the variance of the homogeneous broadening factor (spectral density) and is defined by 


$$
Z^{2}=(\gamma / 2)^{2}+\hat{S}\left(\sigma^{2}+\omega_{m}{ }^{2}\right)
$$

where $\sigma^{2}$ is the variance of the distribution of phonons. $\gamma$ is the homogeneous broadening due to effects other than electron-phonon coupling, e.g. pure electronic dephasing. With the mean phonon frequency approximation, $\hat{S}$ is $S \cdot \operatorname{ctnh}\left(\hbar \omega_{m l} / 2 k T\right)$, where $\omega_{\mathfrak{m}}$ is the mean phonon frequency and $S$ is the Huang-Rhys factor for the phonons, which characterizes the electronphonon coupling strength. $\omega_{i}$ and $\mathrm{FC}_{i}$ are the frequency and the $\mathrm{FC}$ factor for the $i^{\text {th }}$ intramolecular vibration mode, respectively. The term $\left(1-e^{-S}\right)$ is the Franck-Condon factor for the phonons, which are taken to be delocalized. In Ref. [39], the electron-phonon coupling parameters for the $B 800 \mathrm{~S}_{0} \rightarrow \mathrm{Q}_{\mathrm{y}}$ optical transition were obtained (from $\mathrm{HB}$ spectra) as $\omega_{\mathrm{m}} \sim 30 \mathrm{~cm}^{-1}, S \sim 0.3$ and a phonon profile width of $\sim 30 \mathrm{~cm}^{-1}$. Note that the homogeneous broadening, $\gamma$, for LH2 complex was estimated to be $\sim 200 \mathrm{~cm}^{-1}$ [28]. For explanations and physical meaning of FC factors, see Chapter 2.

\subsection{Antenna Complex (FMO complex) of Green Sulfur Bacteria}

The phototropic green sulfur bacteria contain BChl $c$ or $d$ and the carotenoids chlorobactene and $\mathrm{OH}$-chlorobactene as their light-harvesting pigments [47]. Early work on green sulfur bacteria focused on two aspects of their peculiar photosynthetic apparatus: chlorosomes, which contain 900-4500 BChl $c, d$ or $e$ molecules [48], and a BChl $a$ protein. The water-soluble BChl $a$-protein complex, commonly referred to as FMO (FennaMatthews-Olsen) complex, which is located between the chlorosomes and the intramembrane antenna complex, was the first structurally resolved photosynthetic pigment-protein complex [49]. 


\subsubsection{Structure of FMO Complex from Prosthecochloris aestuarii}

The three-dimensional structure of the FMO complex, isolated from green sulfur bacterium Prosthecochloris aestuarii, was first determined by X-ray crystallography [50] (see Figures 1.8 and 1.9 for its structure). More recently, the structure was refined to $1.9 \AA$ resolution [51] and the structure of the FMO complex from Chlorobium tepidum was resolved at a resolution of $2.2 \AA$, which revealed the same main structural features, including all pigment-binding residues, as the FMO complex from $P$. aestuarii [52]. The crystal structures revealed that the $\mathrm{BChl} a$ molecules are bound, within a cylinder of mainly $\beta$-sheet structure, in groups of 7 onto $42 \mathrm{kDa}$ polypeptides that assemble as trimers. An individual FMO trimer in the crystal structure exhibits $\mathrm{C}_{3}$ symmetry and belongs to the $\mathrm{P} 6_{3}$ space group, with cell dimensions $a=b=111.9 \AA$ and $c=98.6 \AA$ [53]. Each subunit within a trimer contains a $\beta$-sheet with 16 strands, enclosing seven pentacoordinate $\mathrm{BChl} a$ pigments. Six out of the 7 symmetry-inequivalent BChls are liganded by protein residues in the interior. BChls $1,3,4,6$, and 7 coordinated to a His residue and BChl 5 is coordinated to a Leu sidechain, whereas $\mathrm{BChl} 2$ is liganded to water [54]. Note that the BChl numbering scheme follows the one given in Ref. [54]. The nearest-neighbor distances within one subunit vary from $11.3 \AA$ to $14.4 \AA$, while the distance is about $24 \AA$ between nearest neighbors in different subunits of the trimer.

\subsubsection{Energy Transfer and Spectral Dynamics of the Three Lowest Energy State}

Excitation energy transfer (EET) processes in the FMO complex occur with time constants that range between tens of $\mathrm{ps}$ and hundreds of $\mathrm{ps}$, vide infra. Theoretical calculation of the rates required a firm understanding of the excitonic level structure. In this 
(a)

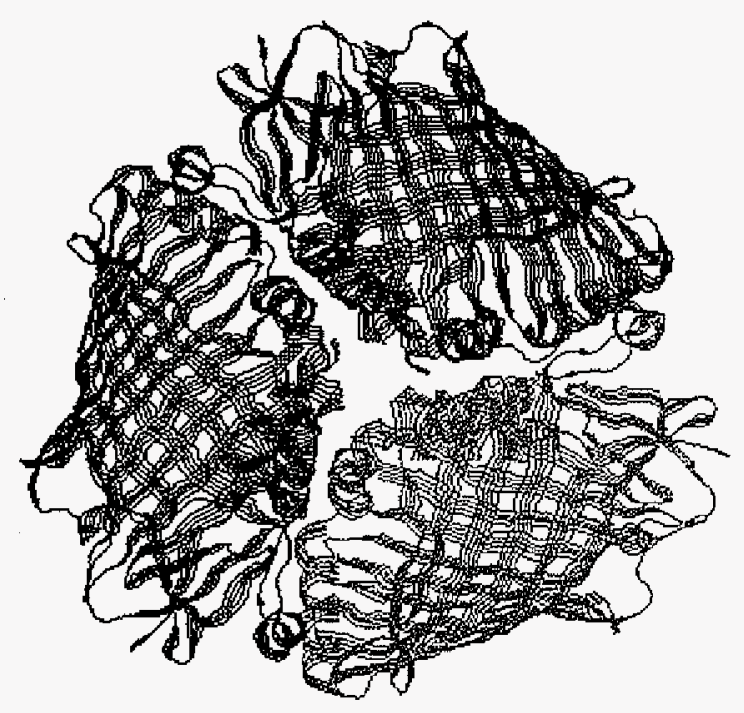

(b)

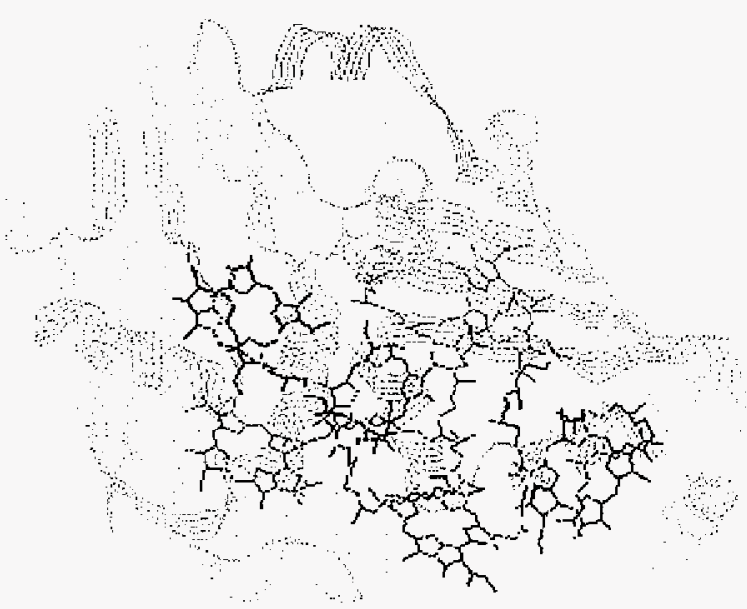

Figure 1.8 Part (a) shows arrangement of the FMO complex trimer from

Prosthecochloris aestuarii, which has a three-fold symmetry. In Part (b), one subunit of the FMO complex showing seven BChls enclosed within an envelope of protein is described. The figures were generated by the program RasMol [15]. 


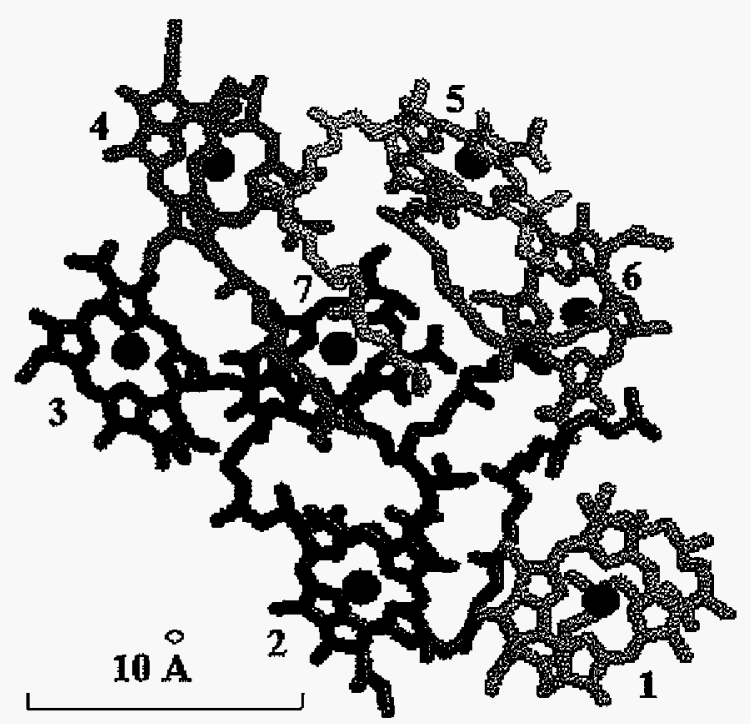

Figure 1.9 Arrangement of the 7 BChls in a subunit of FMO complex (trimer) from Prosthecochloris aestuarii. Blue dots shown in the center of BChls are $\mathrm{Mg}$ ions. The $\mathrm{BChl}$ numbering is given according to the scheme of Matthews and Fenna [54]. The nearest neighboring $\mathrm{Mg}-\mathrm{Mg}$ distances are $11-15 \AA$ and the phytyl chains are packed in the central space between the BChls to form a hydrophobic core. The figure was adapted from Ref. [53]. 
regard, progress has been hampered by the inability to accurately determine the $S_{0} \rightarrow S_{2}\left(Q_{y}\right)$ excitation energies of the 7 symmetry inequivalent $\mathrm{BChl} a$ molecules. However, significant progress has been made in recent years. See below.

It has been postulated that the energy transfer between the $\mathrm{BChl} a$ molecules, spaced at an average of $12 \AA$, could be described on the basis of an exciton model [55]. The first simulations, in particular of the absorption and circular dichroism (CD) spectra, were performed by Pearlstein and coworkers. From the orientations of the BChl $a$ molecules and of their transition moments, the pairwise dipolar interactions in the FMO complex were calculated; the strongest coupling within the subunit is about $200 \mathrm{~cm}^{-1}$. It was concluded that the spectra are determined not only by the excitonic coupling between the BChls, which lead to excitonic shifts, but also by the BChls possessing different site energies due to different protein environments $[56,57]$. EET at room temperature of isolated FMO complexes was also studied with subpicosecond resolution. The isotropic decays were characterized by time constants ranging from 100 to $900 \mathrm{fs}$, whereas the anisotropy decays with time constants of $75-135 \mathrm{fs}$ and $1.4-2.0 \mathrm{ps}[58,59]$. Later, measurements at $10 \mathrm{~K}$ showed that the lifetimes of the states at $804,812,815$ and $823 \mathrm{~nm}$ are $0.5,1.7,5.5$ and $37 \mathrm{ps,}$, respectively $[60,61]$. At 10 $\mathrm{K}$, these lifetimes are determined by downward EET. The time constant for the component at the very red edge of the $\mathrm{Q}_{\mathrm{y}}$-absorption spectrum due to the lowest energy state was also determined as $>800 \mathrm{ps}$, due to decay to the ground state [61]. The $\mathrm{Q}_{\mathrm{y}}$-absorption spectra of the FMO complexes from $P$. aestuarii and C. tepidum at $\sim 4 \mathrm{~K}$ are presented in Figure 1.10. Hole-burning experiments in the $\mathrm{Q}_{\mathrm{y}}$-absorption spectrum of the FMO complex at $4 \mathrm{~K}$ led to time constants for total dephasing of $>20 \mathrm{ps}$ in the lowest energy $825 \mathrm{~nm}$ band and about 100 


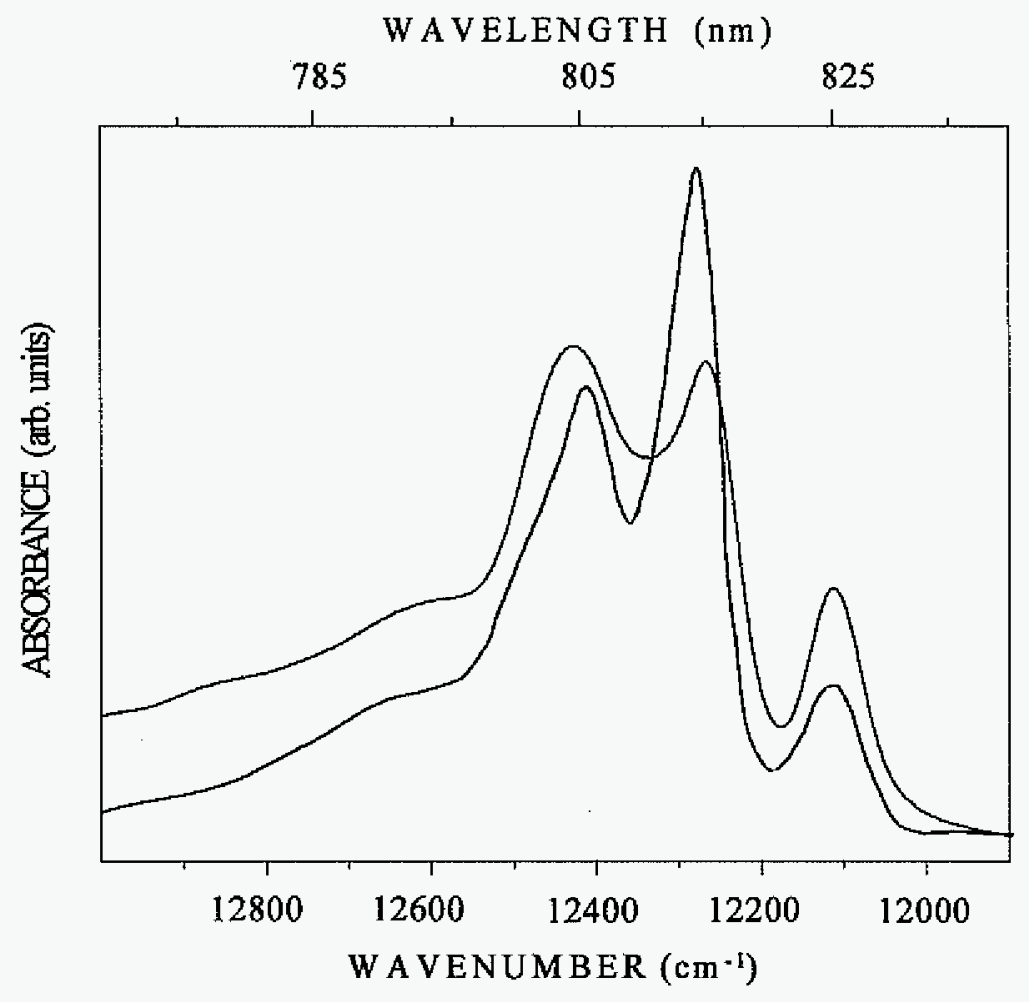

Figure 1.10 The 4.2 K Qy-absorption spectra of the FMO complexes from P. aestuarii (black curve) and C. tepidum (blue curve). 
fs for $\lambda<820 \mathrm{~nm}[62,63]$. Also, the hole-burning studies showed the existence of at least 8 excitonic bands [63].

More recently, optical spectra of the FMO complex from $P$. aestuarii and $C$. tepidum (linear dichroism (LD), triplet-singlet absorption difference, circular dichroism (CD) and ultra-fast pump-probe) led to detailed simulations for the subunit, which used the dipolar interaction energies $[64,65]$. The excitonic Hamiltonian used was [66]

$$
\hat{H}=\sum_{j} E_{j}|j\rangle\langle j|+\sum_{j<i} V_{i j}(|j\rangle\langle i|+| i\rangle\langle j|),
$$

where $E_{j}$ is the site energy of pigment $j$ and $V_{i j}$ is the dipole-dipole interaction between the $i$ th and $j$ th $\mathrm{BChl}$. Note that the excitonic simulations assumed that only $\mathrm{BChl}-\mathrm{BChl}$ interactions within a single subunit are significant. This appears to be quite reasonable since the coupling energies between $\mathrm{BChls}$ belonging to different subunits of the trimers are only a few $\mathrm{cm}^{-1}$. Satisfactory fitting of the various spectra was achieved for both $P$. aestuarii and C. tepidum. The resulting excitonic energies and $\mathrm{S}_{0} \rightarrow \mathrm{Q}_{\mathrm{y}}$ dipolar strength (Debye ${ }^{2}$ ) are given in Table 1.1. The last column gives the $\mathrm{BChl}(\mathrm{s})$ that contributes most significantly to the excitonic state.

From the above simulations and other results (vide infra), it was concluded that the $825 \mathrm{~nm}$ absorption band is contributed to by three trimer states which stem from the lowest energy $\mathrm{Q}_{\mathrm{y}}$-state of the subunit highly localized on a single BChl $a$ molecule (most likely BChi 3) $[64,67]$. For perfect $C_{3}$ symmetry, there should be a doubly degenerate $E$ state and non-degenerate A state. However, energy disorder (diagonal and/or off-diagonal) due to the glass-like structural heterogeneity of proteins would split the degeneracy of the $E$ state and mix it with the A state. With BChl 3 responsible for the lowest energy state of the subunit, 
Table 1.1 Simulated excitonic energies and dipole strengths for the FMO complexes P. aestuarii ${ }^{\mathrm{a}}$

\begin{tabular}{|c|c|c|c|}
\hline State & State Energies, $\mathrm{cm}^{-1}(\mathrm{~nm})$ & Dipole Strength, $\mathrm{D}^{2}$ & $\mathrm{BChl}$ \\
\hline 1 & $12111(825.7)$ & 49.7 & 3 \\
\hline 2 & $12278(814.5)$ & 138.8 & 4 \\
\hline 3 & $12312(812.2)$ & 60.6 & 1 \\
\hline 4 & $12410(805.8)$ & 100.3 & 6 \\
\hline 5 & $12488(800.8)$ & 71.1 & 7 \\
\hline 6 & $12566(796.4)$ & 26.7 & 2 \\
\hline 7 & $12610(793.0)$ & 34.6 & 5 \\
\hline
\end{tabular}

C. tepidum ${ }^{\mathrm{b}}$

\begin{tabular}{|c|c|c|c|}
\hline State & State Energies, $\mathrm{cm}^{-1}(\mathrm{~nm})$ & Dipole Strength, $\mathrm{D}^{2}$ & BChl \\
\hline 1 & $12113(825.6)$ & 48.9 & 3 \\
\hline 2 & $12262(815.5)$ & 121.0 & 4 \\
\hline 3 & $12355(809.3)$ & 79.5 & 1 \\
\hline 4 & $12414(805.6)$ & 92.3 & 5,6 \\
\hline 5 & $12448(803.3)$ & 59.8 & 7 \\
\hline 6 & $12611(793.0)$ & 27.6 & 5,6 \\
\hline 7 & $12649(790.9)$ & 52.2 & 2 \\
\hline
\end{tabular}

${ }^{a}$ From simulation results described in Ref. [66].

${ }^{\mathrm{b}}$ From Ref: [65]. 
the A state is weakly absorbing in the absence of energy disorder [64,67]. Temperature dependent (1.8-10 K) non-photochemical hole-burning (NPHB) and zero-phonon hole growth kinetics measurements on C. tepidum showed that the $825 \mathrm{~nm}$ band is contributed to by 3 states at $\sim 823,825$ and $827 \mathrm{~nm}$ with comparable absorption intensities and a static inhomogeneous broadenings of $\sim 50 \mathrm{~cm}^{-1}$ [68]. The $2 \mathrm{~nm}$ gaps were considered to be due to strong diagonal energy disorder stemming from structural disorder.

Both the low temperature photon echo experiments on FMO complex of P. aestuarii [69] and the NPHB on FMO complex of C. tepidum [68] showed that the lifetimes of the states excited at high energy side of the $825 \mathrm{~nm}$ band are significantly shorter than that of the state excited at the low energy side. The lifetime values obtained by hole-burning were 117 and $37 \mathrm{ps}$ for burning wavelengths equal to 823 and $825 \mathrm{~nm}$ [68], while that of the lowest state was $\sim 2$ ns. The 117 and 37 ps lifetimes were attributed to downward EET between the three lowest states. (Note that pure dephasing/spectral diffusion contribution due to the glass-like two-level systems of the protein needed to be taken into account in the interpretation of the temperature dependencies $(1.8-10 \mathrm{~K})$ of the $\mathrm{ZPH}$ holewidths. See Section 2.2 for more details on pure dephasing.)

As will be seen in Chapter 3, the hole spectra of the FMO complex exhibit unusually rich low energy satellite structure. The theoretical simulations of the hole spectra revealed that these satellite holes are due to intermolecular protein phonons and low energy intramolecular vibrations of $\mathrm{BChl} a$. A simulated single-site absorption profile and absorption spectrum that takes into account these modes are presented in Figure 1.11. The simulation is based on the theory described in Section 2.3 .2 and performed using the program presented in the Appendix. Readers interested in the simulation should refer to 
(a)

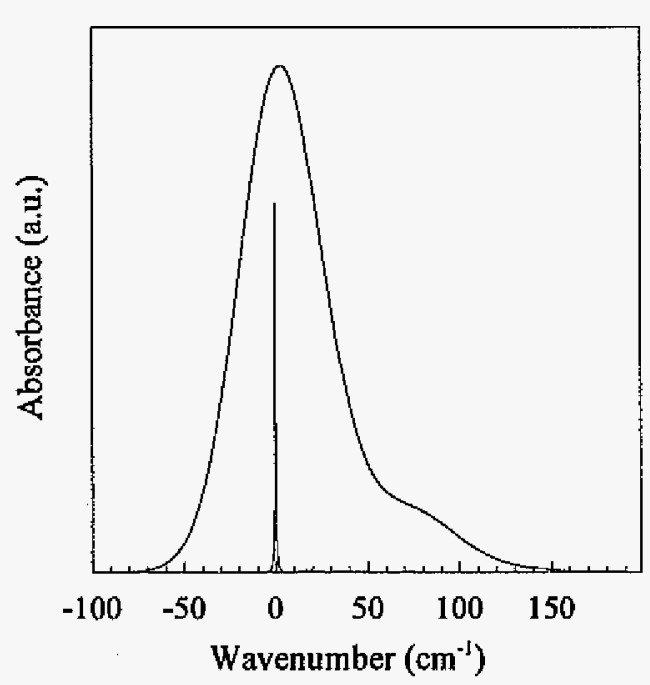

(b)

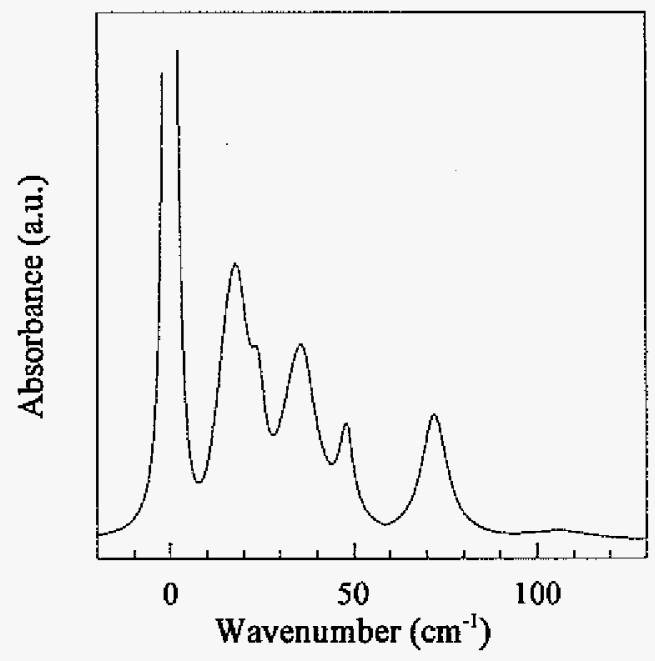

Figure 1.11 Part (a) shows the simulated single-site absorption profile (5-mode) and absorption spectrum, while Part (b) shows a magnified view of the low frequency vibrational sideband structure of the single-site profile for clarification. The simulation parameters used are $\omega\{1 . .5\}=18,24,36,48$ and $72 \mathrm{~cm}^{-1}$ and $S\{1 . .5\}=0.24,0.05,0.12,0.04$ and 0.12 , with bandwidths of 10,5 , 10,5 and $7.5 \mathrm{~cm}^{-1}$, respectively, as given in Chapter $3 . \Gamma_{\mathrm{inh}}=50 \mathrm{~cm}^{-1}$ was used to generate the absorption spectrum. 
corresponding sections for further discussions. The parameter values for the simulation are given in the figure caption. See Chapter 3 for more details on the low energy satellite structure.

\section{References:}

1. Wraight, C. in Photosynthesis (Govindjee ed.), 1982, Academic Press, pp. 17-61.

2. Hill, R. and Scarisbrick, R. Nature 1940, 146, 61 .

3. Ruben, S., Randall, M., Kamen, M. and Hyde, J. L. J. Am. Chem. Soc. 1941, 63, 877.

4. Frenkel, A.W. Photosynth Res. 1995, 46, 73.

5. Duysens, L. N. M. Photosynth Res. 1989, 21, 61 .

6. Witt, H. T. Photosynth Res. 1991, 29, 55.

7. Mitchell, P. Nature 1961, 191, 144.

8. Deisenhofer, J. and Michel, H. in The Photosynthetic Reaction Center (Vol. II, Deisenhofer, J. and Norris, J. R. eds.), 1993, Academic Press, pp. 541-574.

9. Deisenhofer, J., Epp, O., Miki, K., Huber, R. and Michel, H. J. Mol. Biol. 1984, 180, 385 .

10. Deisenhofer, J., Epp, O., Miki, K., Huber, R. and Michel, H. Nature 1985, 318, 618.

11. Sturgis, J. N., Olsen, J. D., Robert, B. and Hunter, C. N. Biochemistry 1997, 36, 2772.

12. Zuber, $\mathrm{H}$. in Antennas and Reaction Centers of Photosynthetic Bacteria (Springer Series in Chemical Physics Vol.42, Michel-Beyerle, M. E. ed.), 1985, SpringerVerlag, pp. 2-14.

13. Zuber, H. Photochem. Photobiol. 1985, 42, 821.

14. Miller, K, R. Nature 1982, 300, 53.

15. Humphrey, W. F., Dalke, A. and Schulten, K. J. Mol. Graphics 1996, 14, 33. 
16. Schulten, K. in Simplicity and Complexity in Proteins and Nucleic Acids (Fauenfelder, H., Deisenhofer, J. and Wolynes, P. G. eds.), 1999, Dahlem University.

17. McDermott, G., Prince, S. M., Freer, A. A., Hawthornthwaite-Lawless, A. M., Papez, M. Z., Cogdell, R. J. and Isaacs, N. W. Nature 1995, 274, 517.

18. Freer, A., Prince, S., Sauer, K., Papiz, M., Hawthornthwaite-Lawless, A., McDermott, G., Cogdell, R. J. and Isaacs, N. W. Structure 1996, 4, 449.

19. Sauer, K., Cogdell, R. J., Prince, S. M., Freer, A. A., Isaacs, N. W. and Scheer, H. Photochem. Photobiol. 1996, 64, 564.

20. Krueger, B. P., Scholes, G. D. and Fleming G. R. J. Phys. Chem. B 1998, 102, 2284.

21. Karrasch, S., Bullough, P. A. and Ghosh, R. EMBO J. 1995, 14, 631.

22. Wu, H-. M. and Small, G. J. Chem. Phys. 1997, 218, 225.

23. Wu, H-. M. and Small, G. J. J. Phys. Chem. B 1998, 102, 888.

24. Rätsep, M., Wu, H.-M., Hayes, J. M. and Small, G. J. Spectrochim. Acta Part A 1998, 54, 1279.

25. Hochstraser, R. M. in Molecular Aspects of Symmetry (Benjamin, W. A. ed.), 1966, New York.

26. Wu, H.-M., Rätsep, M., Jankowiak, R., Cogdell, R. J. and Small, G. J. J. Phys. Chem. B 1997, 101, 7641 .

27. Rätsep, M., Wu, H.-M., Hayes, J. M., Blankenship, R. E., Cogdell, R. J. and Small, G. J. J. Phys. Chem. B 1998, 102, 4035.

28. Sundström, V., Pullerits, T. and van Grondelle, R. J. Phys. Chem. B 1999 103, 2327.

29. Jelezko, F., Tietz, C., Gerken, U., Wrachtrup, J. and Bittl, R. J. Phys. Chem. B 2000, 104,8093 .

30. van Oijen, A. M., Ketelaars, M., Köhler, J., Aartsma, T. J. and Schmidt, J. Biophysical J. 2000, 78, 1570.

31. Jimenez, R., Dikshit, S. N., Bradforth, S. E. and Fleming, G. R. J. Phys. Chem. 1996 100,6825 .

32. Wu, H.-M., Reddy, N. R. S., Cogdell, R. J., Muenke, C., Michel, H. and Small, G. J. Mol. Cryst. Liq. Cryst. 1996, 291, 163. 
33. Monshouwer, R., Baltuška, A., van Mourik, F. and van Grondelle, R. J. Phys. Chem. A 1998, 102, 4360 .

34. Kumble, R., Palese, S., Visschers, R. W., Dutton, P. L. and Hochstrasser, R. M. Chem. Phys. Lett. 1996, 261, 396.

35. Hess, S., Chachisvilis, M., Jones, M. R., Hunter, C. N. and Sundstörm, V. Proc. Natl. Acad. Sci. USA 1995, 92, 12333.

36. Chachisvilis, M., Kühn, O., Pullerits, T. and Sundstörm, V. J. Phys. Chem. B 1997, 101,7275 .

37. Kramer, H. J. M., van Grondelle, R., Hunter, C. N., Westerhuis, W. H. J. and Amesz, J. Biochim. Biophys. Acta 1984, 765, 156.

38. Hess, S., Åkesson, E., Cogdell, R. J., Pullerits, T. and Sundstörm, V. Biophys. J. $1995,69,2211$.

39. Wu, H.-M., Savikhin, S., Reddy, N. R. S., Jankowiak, R., Cogdell, R. J., Struve, W. S. and Small, G. J. J. Phys. Chem. 1996, 100, 12022.

40. Ma, Y.-Z., Cogdell, R. J., Gillbro, T. J. Phys. Chem. B 1998, 102, 881.

41. Shreve, A. P. Trautman, J. K., Frank, H. A., Owns, T. G. and Albrecht, A. C. Biochim, Biophys. Acta 1991, 1058, 280.

42. Scholes, G. D. and Fleming G. R. J. Phys. Chem. B 2000, 104, 1854.

43. Mukai, K, Abe, S. and Sumi, H. J. Phys. Chem. B 1999, 103, 6069.

44. Kolaczkowski, S. V., Hayes, J. M. and Small, G. J. J. Phys. Chem. 1994, 98, 13418.

45. Reddy, N. R. S., Wu H.-M., Jankowiak, R., Picorel, R., Cogdell, R. J. and Small, G. J. Photosynth. Res. 1996, 8, 277.

46. Wu, H.-M. Ph.D. Dissertation, lowa State University, 1998.

47. Sybesma, C. and Vrendenberg, W. J. Biochim. Biophys. Acta 1963, 75, 439.

48. Olson, J. M. Biochim. Biophys. Acta 1980, 584, 33 .

49. Fenna, R. E. and Matthews, B. W. Nature 1975, 258, 573.

50. Matthews, B. W., Fenna, R. E., Bolognesi, M. C., Schmid, M. F. and Olson, J. M. J. Mol. Biol. 1979, 131, 259-285. 
51. Tronrud, D. E., Schmid, M. F. and Matthews, B. W. J. Mol. Biol. 1986, 188, 443.

52. Li, Y. F., Zhou, W., Blankenship, R. E., Allen, J. P. J. Mol. Biol. 1997, 271, 456.

53. Savikhin, S., Buck, D. R. and Struve, W. S. in Resonance Energy Transfer (Andrews, D. L. and Demidov, A. A. eds.), 1999, John Wiley \& Sons, Chichester, p. 399.

54. Matthews, B. W. and Fenna, R. E. Acc. Chem. Res. 1980, 13, 309.

55. Pearlstein, R. M. and Hemenger, R. P. Proc. Natl. Acad. Sci. U.S.A 1978, 75, 4920.

56. Pearlstein, R. M. Photosynth. Res. 1992, 31, 213.

57. Lu, X. and PearIstein, R. M. Photochem. Photobiol. 1993, 57, 86.

58. Savikhin, S., Zhou, W., Blankenship, R. E. and Struve, W. S. Biophys. J. 1994, 66, 110.

59. Savikhin, S. and Struve, W. S. Biochemistry. 1994, 33, 11200.

60. Savikhin, S. and Struve, W. S. Photosynth. Res. 1996, 48, 271.

61. Vulto, S. I. E., Streltsov, A. M. and Aartsma, T. J. J. Phys. Chem. B 1997, 101, 4845.

62. Johnson, S. G. and Small, G. J. Chem. Phys. Lett. 1989, 155, 371.

63. Johnson, S. G. and Small, G. J. J. Phys. Chem. 1991, 95, 471.

64. Louwe, R. J. W., Vrieze, J., Hoff, A. J. and Aartsma, T. J. J. Phys. Chem. B 1997, 101,11280 .

65. Vulto, S. I. E., de Baat, M. A., Louwe, R. J. W., Permentier, H. P., Neef, T., Miller, M., van Amerongen, H. and Aartsma, T. J. J. Phys. Chem. B 1998, 102, 9577.

66. Vulto, S. I. E., de Baat, M. A., Neerken, S., Nowak, F. R., van Amerongen, H., Amesz, J. and Aartsma, T. J. J. Phys. Chem. B 1999, 103, 8153.

67. Gülen, D. J. Phys. Chem. 1996, 100, 17684.

68. Rätsep, M., Blankenship, R. E. and Small, G. J. J. Phys. Chem. B 2000, 103, 5736.

69. Louwe, R. J. W. and Aartsma, T. J. J. Phys. Chem. 1997, 101, 7221. 


\section{CHAPTER 2. GENERAL INTRODUCTION TO NONPHOTOCHEMICAL HOLE-BURNING SPECTROSCOPY}

\subsection{General Introduction to Hole-burning Spectroscopy}

Fundamental mechanisms and applications of hole-burning spectroscopy and theories related to hole spectra are covered in this chapter. These theories are the foundation for simulations of hole spectra for the FMO complex study (Chapter 3). In addition, molecular exciton theory is briefly described to provide background on the exciton level structure calculations reported in the study of the LH2 complex (Chapter 4).

The optical absorption bands of chromophores in amorphous solid hosts such as glasses and polymers exhibit large inhomogeneous broadening. This is also the case for Chls in proteins. Such broadening is the result of a chromophore experiencing different environments, i.e. interactions between the chromophore and the host molecules gives rise to a distribution of transition frequencies [1]. A schematic illustration of this is depicted in the Figure 2.1. Typical inhomogeneous broadening of electronic absorption bands are $\Gamma_{\text {inh }}$ $\sim 100-300 \mathrm{~cm}^{-1}$ [2]. On the other hand, each particular chromophore carries a finite width, which is called homogeneous width $(\gamma)$ and is determined by the total dephasing time $\left(T_{2}\right)$ associated with the optical transition of the chromophore in its environment. The linewidth of the homogeneous broadening is given by

$$
\gamma\left(\mathrm{cm}^{-1}\right)=\frac{1}{\pi T_{2} c}
$$

where $T_{2}$, the total dephasing time, is defined [3] by

$$
\frac{1}{T_{2}}=\frac{1}{2 T_{1}}+\frac{1}{T_{2}^{*}},
$$


(a)

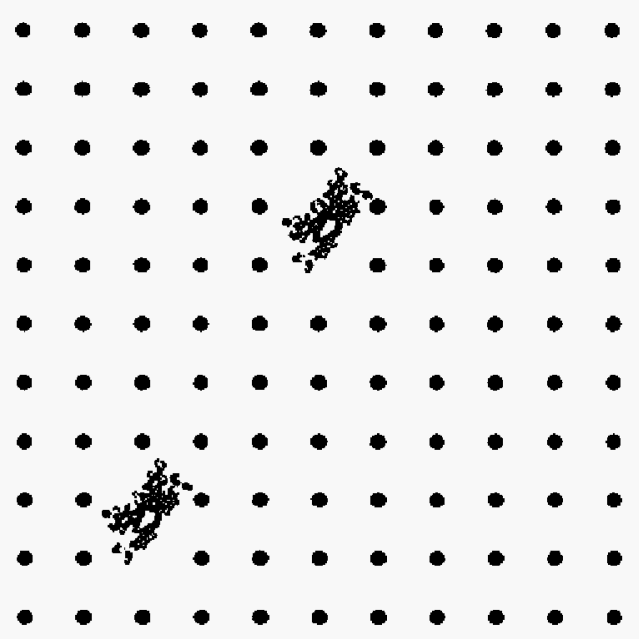

(b)

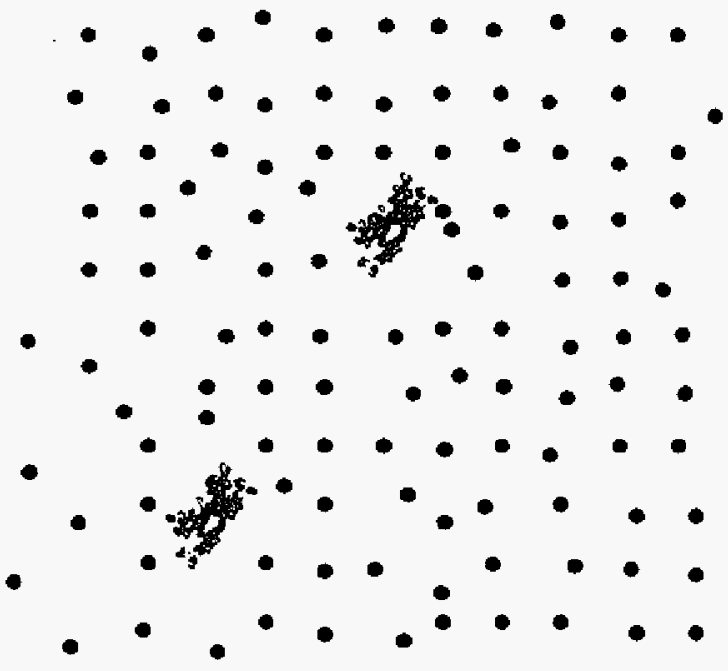

Figure 2.1 Parts (a) and (b) show schematic views of guest molecules in a perfect lattice (host) and in an amorphous lattice, respectively. In Part (a) the ZPLs appear at same transition frequency. However, in Part (b) the ZPLs appear at different frequencies, as shown in Figure 2.2. 
where $T_{1}$ is the excited state lifetime of the chromophore and $T_{2}{ }^{*}$ is the pure dephasing time of the molecules. As seen in Eq. (2), the total optical dephasing time of a chromophore consists of two parts: (1) the excited state lifetime which is usually weakly dependent on temperature and (2) the pure dephasing time, which is due to interaction of the chromophore with the host matrix and, therefore, temperature dependent. Electronic dephasing/spectral diffusion will be briefly discussed in the following section.

Information on a specific subset of chromophores in a particular local environment is well-hidden behind other subsets of absorbers. The use of extremely narrow linewidth lasers enables selective excitation of a spectrally narrow subset of absorbers to circumvent the screening effect of inhomogeneous broadening. Up to date, two main frequency domain classes of selective excitation techniques exist: A. Fluorescence line narrowing spectroscopy (FLNS), which name is meant to indicate that fluorescence spectra obtained under selective excitation conditions can be much narrower than those obtained under non-selective excitation conditions. Description of the FLN mechanism is omitted here since the technique is out of scope of this thesis; B. Spectral hole-burning spectroscopy. Hole-burning is typically a two part process consisting of recording the absorption spectrum before and after burning with a narrow frequency laser. Burning is the result of the irradiation of the amorphous system at the hole-burning frequency $\omega_{\mathrm{B}}$. The hole is observed as a spectrally narrow area of reduced absorption in the absorption band close to $\omega_{\mathrm{B}}$. In general, no matter which mechanism of hole-burning (see Section 2.2) is employed, two conditions are mandatory for hole-burning: (1) the presence of an inhomogeneously broadened absorption band of the chromophore and (2) a persistent transition frequency change mechanism that involves the excited states of the chromophore. 
Selectively exciting specific chromophores results, in part, in a zero-phonon transition. A zero-phonon line (ZPL) is an electronic transition that does not involve a change in the total number of phonons. The ZPL is accompanied by a phonon wing, which is known as a phonon side-band (PSB). The PSB arises due to (linear) electron-phonon coupling between the initial and final electronic states of the chromophore and the phonon bath of the host matrix. The ZPL and PSB together form a single site absorption profile and it, in low temperature limit, is given by $[4,5]$

$$
L(\Omega-v)=\prod_{j=1}^{N} \sum_{R=0}^{\infty} \frac{e^{-S_{j}} S_{j}{ }^{R}}{R !} l_{R}\left(\Omega-v-R \omega_{j}\right)
$$

in harmonic approximation, where $v$ is a ZPL frequency, $l$ is the line shape function and $j$ corresponds to the discrete pseudolocalized or localized phonons and intramolecular vibrational modes. $S_{j}$ is the Huang-Rhys factor, which is a measure of the electron-phonon coupling strength, and $\omega_{j}$ is the center frequency of one-phonon profile with respect to the ZPL of the $j^{\text {th }}$ mode. For $R=0, L$ is the lineshape of the ZPL with a homogeneous width, $\gamma$. Values of $R(R=1,2,3 \ldots)$ correspond to one-, two-, three- ... phonon profiles, which for $\mathrm{R}$ $\geq 1$, are given by the convolution of the one-phonon profile with itself $R$ times. Convolution of a single-site absorption profile with a Gaussian shaped site-distribution function (SDF), whose mean is at $v_{\mathrm{m}}$, gives the inhomogeneously broadened absorption spectrum,

$$
A(\Omega)=\int d v N\left(v-v_{m}\right) L(\Omega-v),
$$

where $N\left(v-v_{m}\right)$ is the probability function of finding a site with a zero-phonon transition frequency at $v$. Figure 2.2 schematically shows an inhomogeneously broadened absorption band and single site absorption profiles, each consisting of a Lorentzian-shaped ZPL and an accompanying PSB. 


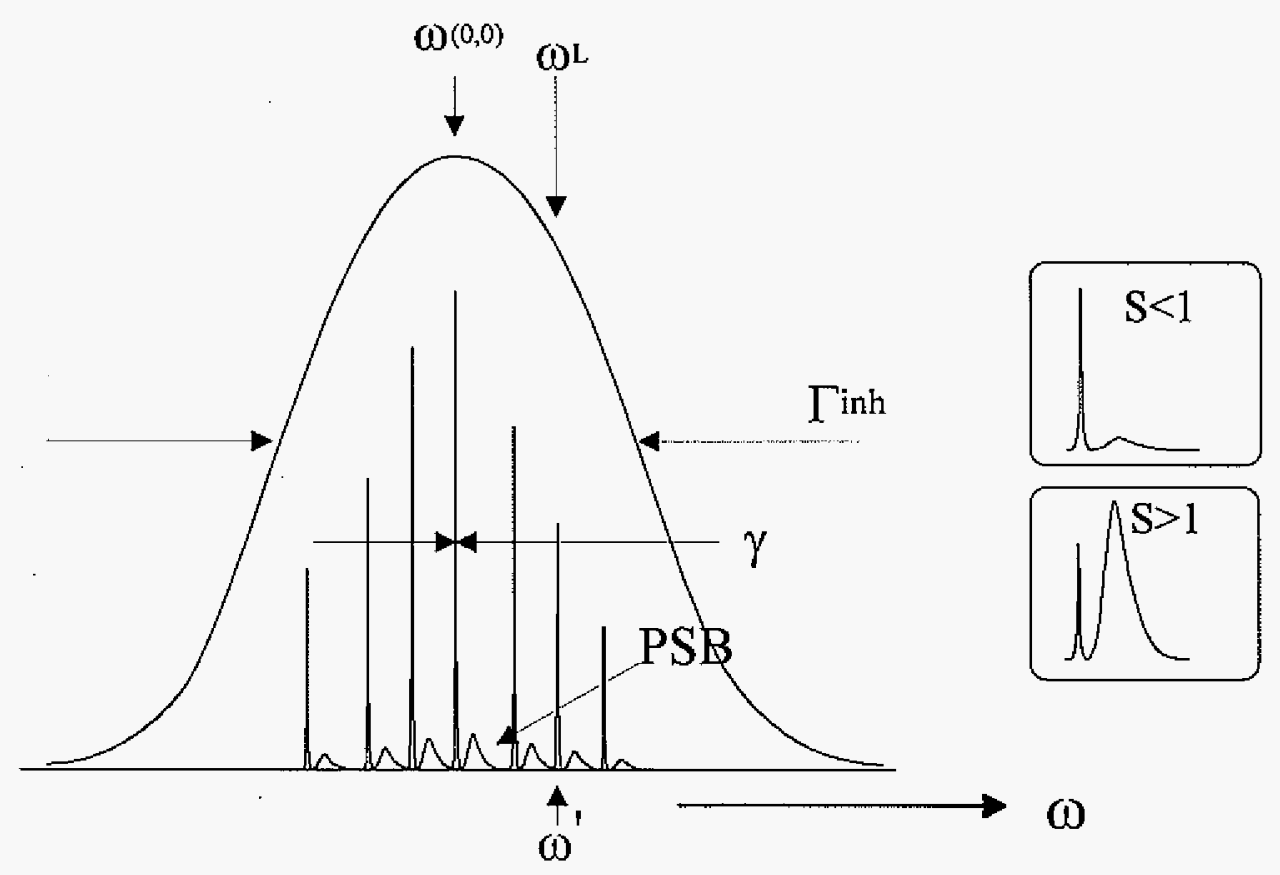

Figure 2.2 Diagram of homogeneously $(\gamma)$ and inhomogeneously $\left(\Gamma_{\text {inh }}\right)$ broadened absorption bands. The ZPLs and their phonon side bands are enlarged for clarification. $\omega_{\mathrm{L}}$ is the laser frequency for selective excitation of a narrow isochromat. The windows at the right describe the schematic difference of the relative ZPL/PSB structures with respect to Huang-Rhys factor (electronphonon coupling strength). Strong and weak coupling are defined by $S>1$ and $\mathrm{S}<1$, respectively. 
In a hole-burning experiment, the reduction of specific chromophores forms a zerophonon hole ( $\mathrm{ZPH})$ and an accompanying phonon-sideband hole (PSBH). The width of the $\mathrm{ZPL}$ can be obtained from the ZPH width, $\Gamma_{\mathrm{h}}$, and it is given by

$$
\Gamma_{\mathrm{h}}=2 \gamma
$$

Note that lasers applied in the candidate's experiments had a linewidth that was typically more than ten times narrower than $\Gamma_{\mathrm{h}}$. The hole profile at burning time $\tau$ is derived from $A_{\tau}(\Omega)-A_{0}(\Omega)$ where $A_{\tau}(\Omega)$ and $A_{0}(\Omega)$ stand for the absorption spectrum after and before burning, respectively (see section 2.3 .2 for details).

Spectral hole-burning has been applied to photosynthetic protein-pigment complexes over the past decade [6-9]. The width of the $\mathrm{ZPH}$, which coincides with the burn frequency $\left(\omega_{B}\right)$, was utilized to determine lifetimes of excited states, which corresponds to the inverse rate of electron transfer (ET) or excitation energy transfer (EET), as well as to resolve closely spaced exciton levels. Pure dephasing of $\mathrm{Q}_{\mathrm{y}}$-optical transitions due to coupling with bistable configurations of the protein (in two-level system) can also be determined, see Section 2.2. Moreover, the linear electron-phonon coupling can be completely determined [7,9]. According to hole-burning theory [6], the Huang-Rhys factor, $S$, is obtainable from the integrated intensity of the real-PSBH divided by that of $\mathrm{ZPH}$ at $\lambda_{\mathrm{B}}$ (burning wavelength). However, with nonphotochemical hole-burning, the blue-shifted anti-hole, usually from the pseudo-PSBH, interferes with the real-PSBH. $S$ can also be determined using the fact that the saturated fractional depth of the ZPH is equal to $\exp (-S)$, which is the Franck-Condon factor for the ZPL $[10,11]$. Normally, intense ZPLs are observed for weak electron-phonon coupling $(S<1)$ cases (see Figure 2.2). Most of the hole-burning studies of the photosynthetic complexes exhibit weak electron-phonon coupling for antennae $[6,8,9]$. The 
hole spectra of bacterial antenna complexes often show low-frequency vibrational modes, in addition to those characteristic of weak electron-phonon coupling, as seen in Chapter 3.

Spectral hole-burning also provides valuable information about inhomogeneous broadening. In an action spectrum, the ZPHs are burned under constant burn fluency conditions across the absorption band of interest $[12,13]$. Assuming that the nonphotochemical hole-burning (NPHB) efficiency is independent of the burn fluency, the packets of ZPHs describe the inhomogeneously broadened absorption band. Nonphotochemical ZPH action spectroscopy has often been used to probe weakly absorbing states that contribute to an absorption band (see Chapter 4). In hole-burning, because of the inherent amorphous structural disorder of the chromophores, the action spectrum reveals the extent of heterogeneity, which is responsible for site inhomogeneous broadening, $\Gamma_{\text {inh }}$ of an individual absorption band. $\Gamma_{\text {inh }}$ 's of chlorophylls and bacteriochlorophylls in antenna pigment-protein complexes and reaction centers are found to be in the range of $\sim 50-200$ $\mathrm{cm}^{-1}[6-8]$.

More recently, hole-burning techniques combined with high-pressure and/or external electric (Stark) fields have been applied to some photosynthetic antenna complexes to determine the pressure shift rates and the permanent dipole moment changes associated with the $\mathrm{S}_{0} \rightarrow \mathrm{S}_{1}\left(\mathrm{Q}_{\mathrm{y}}\right)$ state: Readers interested in the recent spectral hole-burning studies with external fields effects on the photosynthetic complexes should refer to Refs. [14-18].

\subsection{Hole-burning Mechanisms}

There are three mechanisms for hole-burning spectroscopy [6]. All three have in common a process that leads to an absorption decrease at the frequency $\left(\omega_{\mathrm{B}}\right)$ of the absorbed 
photon. The three types of spectroscopies are referred to as photochemical hole-burning (PHB), nonphotochemical hole-burning (NPHB) and population bottleneck hole-burning, which is not described in this section. PHB can be observed for both amorphous and crystalline hosts and it involves a photoreaction of the chromophore in its excited state, such as tautomerization, bond-breaking, and isomerization. PHB was first observed for free base phthalocyanine in n-octane, whereby selective electronic excitation resulted in an intramolecular proton tautomerization [19].

NPHB, on the other hand, does not require the absorbing chromophore to be photoreactive. Nonphotochemical processes result from a "permanent" change in the environment around the chromophore following completion of the optical excitation cycle. As the host configurations are required to be changed in the case of NPHB, NPHB is generally restricted to glass, polymer and protein hosts, with a few exceptions [9]. NPHB was first observed for perylene and for 9-aminoacridine in an ethanol glass [20]. In NPHB, laser excitation of a guest molecule results in a rearrangement of the microenvironment surrounding the guest molecule. NPHB mechanism is favored in amorphous systems because of their inherent structural disorder that allows for reconfiguration of host-guest interactions. NPHB is a reversible process, i.e. the hole can be filled, e.g. by an increase of temperature. The absorption of the photophysical product, known as an antihole, is formed within the original inhomogeneous absorption.

Hayes and Small [21], using a two-level system (TLS) model, first proposed a mechanism for NPHB in 1978. The schematic diagram is described in Figure 2.3. This model premised that amorphous systems consist of bistable host-guest configurations (TLS) that are described by a static distribution of TLS $[6,22,23]$. As depicted in Figure 2.3, 


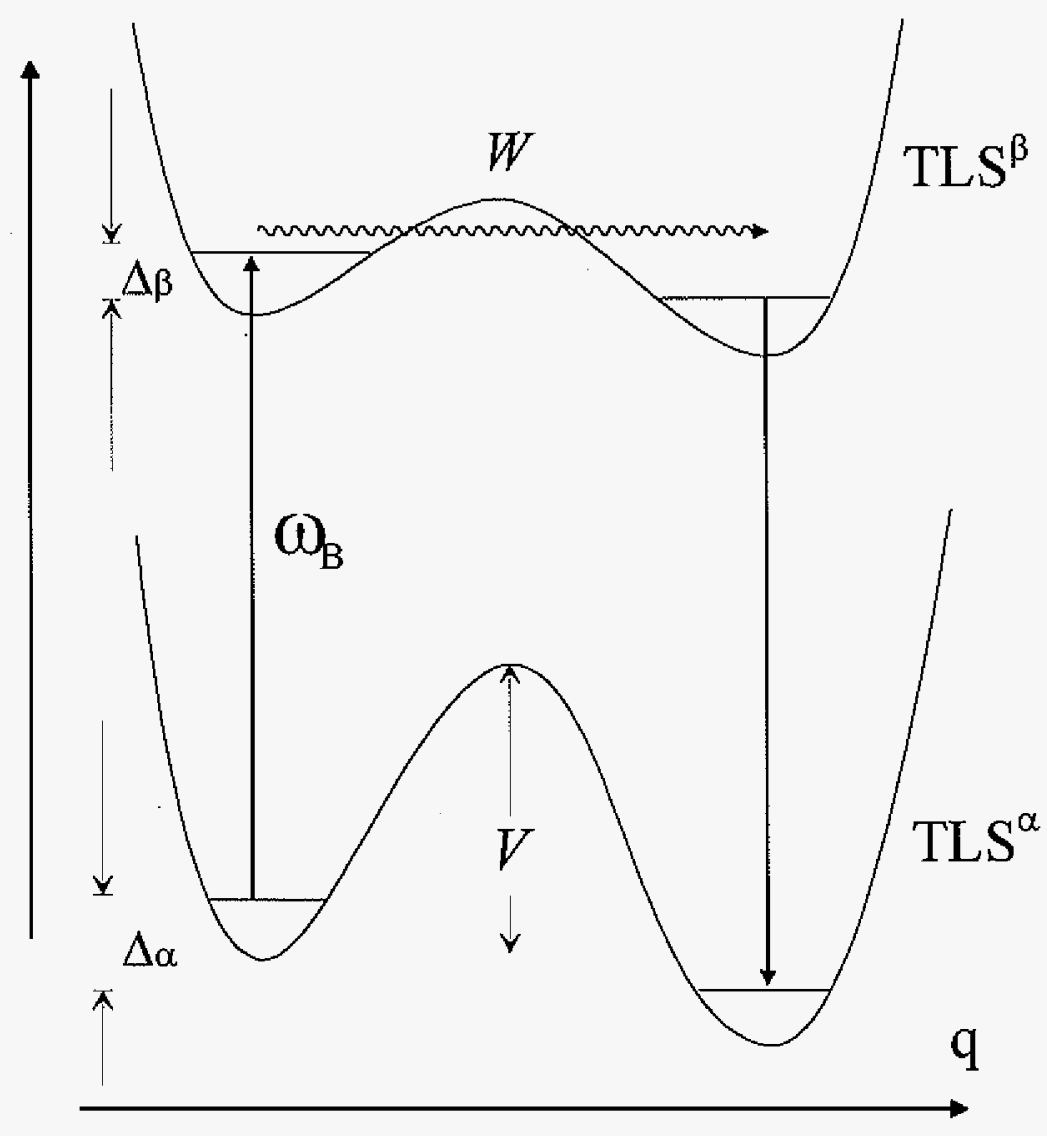

Figure 2.3 Diagram of TLSs coupled to a guest molecule in the ground state $(\alpha)$ and in the excited state $(\beta) . \Delta \alpha$ and $\Delta \beta$ are asymmetry parameters and $q$ represents the intermolecular coordinates. $V$ is the amount of energy barrier and $W$ $\left(=\omega_{0} \exp (-\lambda)\right)$ is the tunneling frequency. $\omega_{\mathrm{B}}$ stands for the excitation frequency. See text for details. 
excitation at the frequency $\omega_{\mathrm{B}}$ is followed by a tunneling process in the excited state, which is typically $\sim 1000$ times more favored than in the ground state, and finally relaxation to the ground state on the right. The tunneling frequency $\mathrm{W}=\omega_{0} \exp (-\lambda)$, where $\lambda$ is the tunnel parameter equals to $\mathrm{d}(2 \mathrm{~m} V)^{1 / 2} / \hbar, V$ is a barrier height, $\mathrm{m}$ is the effective mass of the tunneling entity and $\omega_{0}$ is the harmonic frequency. Since the TLS are asymmetric double well potentials, the new absorption frequency is different, leading to the absence of absorption at $\omega_{\mathrm{B}}$. In 1981, Hayes et al. [24] suggested that two types of TLS are involved in NPHB. The extrinsic TLSs (TLS $\mathrm{ext}_{\mathrm{e}}$ ) were supposed to be strongly associated with the absorbing centers and are responsible for initiation of hole formation. The intrinsic TLSs $\left(\right.$ TLS $\left._{\text {ind }}\right)$ of the host are connected with the excess free volume of glasses and were proposed to cause optical dephasing $[9,21]$. The chromophore (guest) initiates TLS $_{\text {ext }}$ that are associated with the chromophore and its inner shell of solvent molecules. NPHB occurs primarily in amorphous matrices due to a rearrangement of the host environment, triggered by the electron-TLS $\mathrm{S}_{\mathrm{ext}}$ coupling. It is optical excitation of the chromophore that generates the phonon-assisted tunneling processes that lead to the hole formation. The rate-detemining step for hole formation is the excited state phonon-assisted tunneling of $\operatorname{TLS}_{\mathrm{ext}}$.

Later, it was proposed [25] that NPHB is the result of a hierarchy of configurational tunneling events that begin in the outer shell and involve the faster relaxing TLS int $_{\text {, }}$ and terminate in the inner shell. This "outside-in" hierarchy of events causes the reduction of excess free volume in the outer shell (due to TLS $S_{\text {int }}$ tunneling) and leads to an increase in the inner shell free volume about the chromophore (see Figure 2.4 for scheme). NPHB in the inner shell is then the result of prior relaxation events that allow for hole-burning in the limit of $\mathrm{T} \rightarrow 0 \mathrm{~K}$. Moreover, the increase in the free volume for the probe in its inner shell of host 


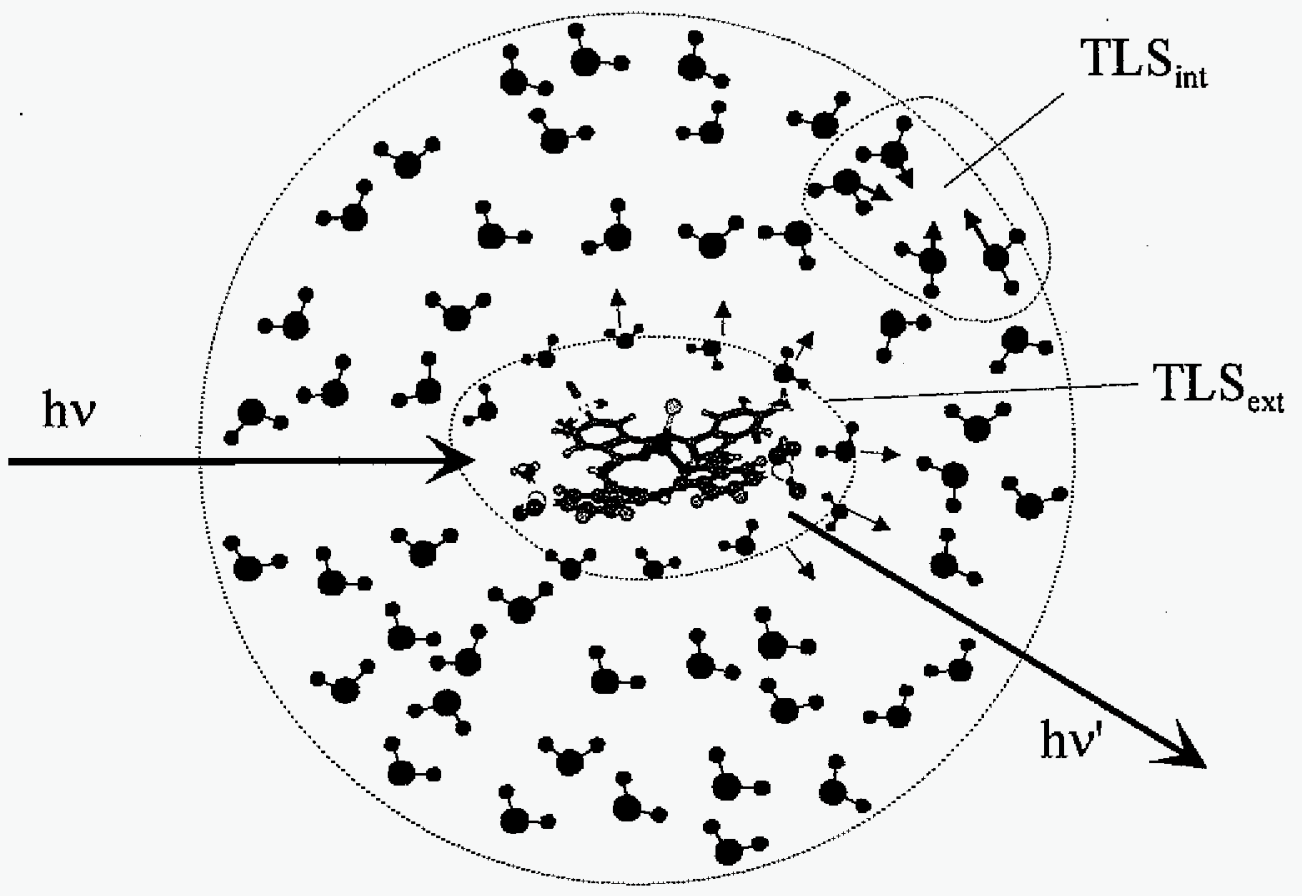

Figure 2.4 Schematic view of NPHB mechanism. See text for discussion. Original figure was prepared by Dr. Tonu Reinot (Dept. of Chem., Iowa State University). 
molecules explains the blue-shifted antihole associated with ${ }^{1} \pi \pi^{*}$ states since such states exhibit a red shift in going from the gas to condensed phase.

Experimentally, spectral holes are measured in fluorescence excitation mode or transmission mode. Both of methods were utilized for this thesis study; detailed experimental setups can be found in the Experimental sections of Chapter 3 and 4. The theory for dispersive hole growth kinetics of laser dyes in glasses was developed in Ref. [26]. More recently, it was refined for the Al-phthalocyanine tetrasulfonate in hyperquenched glassy water (APT/HGW) system [27], the result being

$$
D(\Omega, t)=1.5 \int d \omega L(\Omega-\omega) G(\omega) \int d \lambda f(\lambda) \int d \alpha \sin \alpha \cos ^{2} \alpha\left(e^{\left.-P \sigma \phi(\lambda) L\left(\omega_{s}-\omega\right) \cos ^{2} \alpha\right)}\right),
$$

which is the fractional hole depth at frequency $\Omega$ following a burn for time $t$ with photon flux $P$, where $\omega$ is ZPL frequency, $L(\Omega-\omega)$ is the single site absorption spectrum, $G(\omega)$ is the site excitation energy distribution function, $\sigma$ is the integrated absorption cross-section and $f(\lambda)$ is the normalized Gaussian distribution function for the TLS $S_{\mathrm{ext}}$ tunnel parameter, centered at $\lambda_{0}$. The NPHB quantum yield, $\phi(\lambda)$, is given by

$$
\phi(\lambda)=\Omega_{0} \exp (-2 \lambda)\left[\Omega_{0} \exp (-2 \lambda)+\tau_{\mathrm{fl}}\right]^{-1},
$$

where $\tau_{\mathrm{f}}$ is the fluorescence lifetime. For a detailed discussion, the reader should refer to Ref. [27]. Moreover, as screening processes in NPHB, spontaneous hole filling (SPHF) [28] and light-induced hole filling (LIHF) [29] also need to be considered for more precise hole burning rate analysis. Besides the above factors, the temperature dependence of electronic dephasing in glasses, which can be given by the Jackson-Silbey expression [30] as

$$
\Gamma-\Gamma_{0}=\alpha T^{\alpha}+b_{1} \bar{n}\left(\omega_{1}\right)+b_{2} \bar{n}\left(\omega_{2}\right)
$$


where $\Gamma$ is half the measured holewidth and $\Gamma_{0}$ is the lifetime limited width of the ZPL, has been studied thoroughly. $\bar{n}=[\exp (\hbar \omega / k T)-1]^{-1}$ is the phonon thermal occupation number. $\alpha T^{\alpha}$ describes dephasing from electron-TLS $\mathrm{S}_{\mathrm{int}}$ coupling and the remaining two terms on the right-hand side of Eq. (8) are from exchange coupling of pseudolocalized modes. For a variety of probe molecules in organic glasses, the values of $\alpha$ are $\approx 1.3[3,22,31,32]$. These dephasing studies characterize the quadratic electron-phonon coupling, whereas aforementioned measurements of the frequencies and intensities of the phonons characterize the linear electron-phonon coupling. More recent results of the spectral diffusion in organic glasses are described in Refs. [33,34].

The reader interested in the most recent NPHB mechanism involving multilevel extrinsic systems $\left(\mathrm{MLS}_{\mathrm{ext}}\right.$ ) should refer to the recent review by Reinot et. al [35].

\subsection{Background Theories for Excitation Energy Transfer (EET)}

\subsubsection{Electron-Phonon Coupling}

For the candidate's study, electron-phonon coupling is one of the central terms. This coupling determines the Franck-Condon factors that enter into the nonadiabatic rate expression for EET / electron transfer. Marcus [36-38], Jortner [39] and Hopfield [40] have developed nonadiabatic rate expressions for weak electronic coupling. The rate of a transition from an initial state I to a final state $\mathrm{F}$ is given by the Fermi golden rule [41]

$$
W_{1 \rightarrow \mathrm{F}}=\frac{2 \pi}{\hbar} V^{2}(\mathrm{FC})
$$

where $V$ is the transition matrix element between the two states and FC denotes the spectral density that is determined by Franck-Condon factors. In the harmonic approximation, the 
reorganization energy $(\Lambda)$, the amount of energy required to move the system from an initial equilibrium coordinate $\mathrm{x}_{\mathrm{A}}$ to a final coordinate $\mathrm{x}_{\mathrm{B}}$ is given by

$$
\Lambda=V_{\mathrm{F}}\left(\mathrm{x}_{\mathrm{A}}\right)-V_{\mathrm{I}}\left(\mathrm{x}_{\mathrm{A}}\right)+\Delta E=\frac{\mathrm{k}_{\mathrm{H}}}{2}\left(\mathrm{x}_{\mathrm{A}}-\mathrm{x}_{\mathrm{B}}\right)^{2}
$$

The Huang-Rhys factor, $S$, which is a measure of the electron-phonon coupling strength, is $\Lambda / \hbar \omega$, thus

$$
S=\frac{\mathrm{k}_{\mathrm{H}}}{2 \hbar \omega}\left(\mathrm{x}_{\mathrm{A}}-\mathrm{x}_{\mathrm{B}}\right)^{2},
$$

where $\omega$ is the angular vibrational frequency. See Fig. 2.5 .

The overlap integral, $C\left(n, n^{\prime}\right)$, between the vibrational state $n^{\prime}$ in $\mathrm{F}$ and its corresponding state $n$ in I, defines the probability of the transition from state I to $\mathrm{F}$ and is given by

$$
C^{2}\left(n, n^{\prime}\right)=\left[\int \psi_{n}(\mathrm{x}) \psi_{n^{\prime}}(\mathrm{x}) d \mathrm{x}\right]^{2}
$$

where $\psi_{n}(\mathrm{x})$ is the vibrational wavefunction for state $n$ and $\mathrm{x}$ is the oscillator coordinate. $C^{2}\left(n, n^{\prime}\right)$ is the Franck-Condon factor. Then, the FC factor for the final state $n^{\prime}$ is given by

$$
\mathrm{FC}=\sum_{n=0}^{\infty} C^{2}\left(n, n^{\prime}\right) F(n, T)
$$

where $F(n, T)$ is the fraction of systems in state $n$ at temperature $T$ and is given by a Boltzman distribution. The exact expression for $C^{2}\left(n, n^{\prime}\right)$ in the case of $n^{\prime}=n+p$ has been derived in [42] and it is

$$
C^{2}(n, n+p)=n !(n+p) ! S^{p} e^{-S}\left[\sum_{R=0}^{n} \frac{(-S)^{R}}{R !(n-R) !(p+R) !}\right]
$$

In the low temperature limit $(T \rightarrow 0 \mathrm{~K}), n=0$ and resulting in 


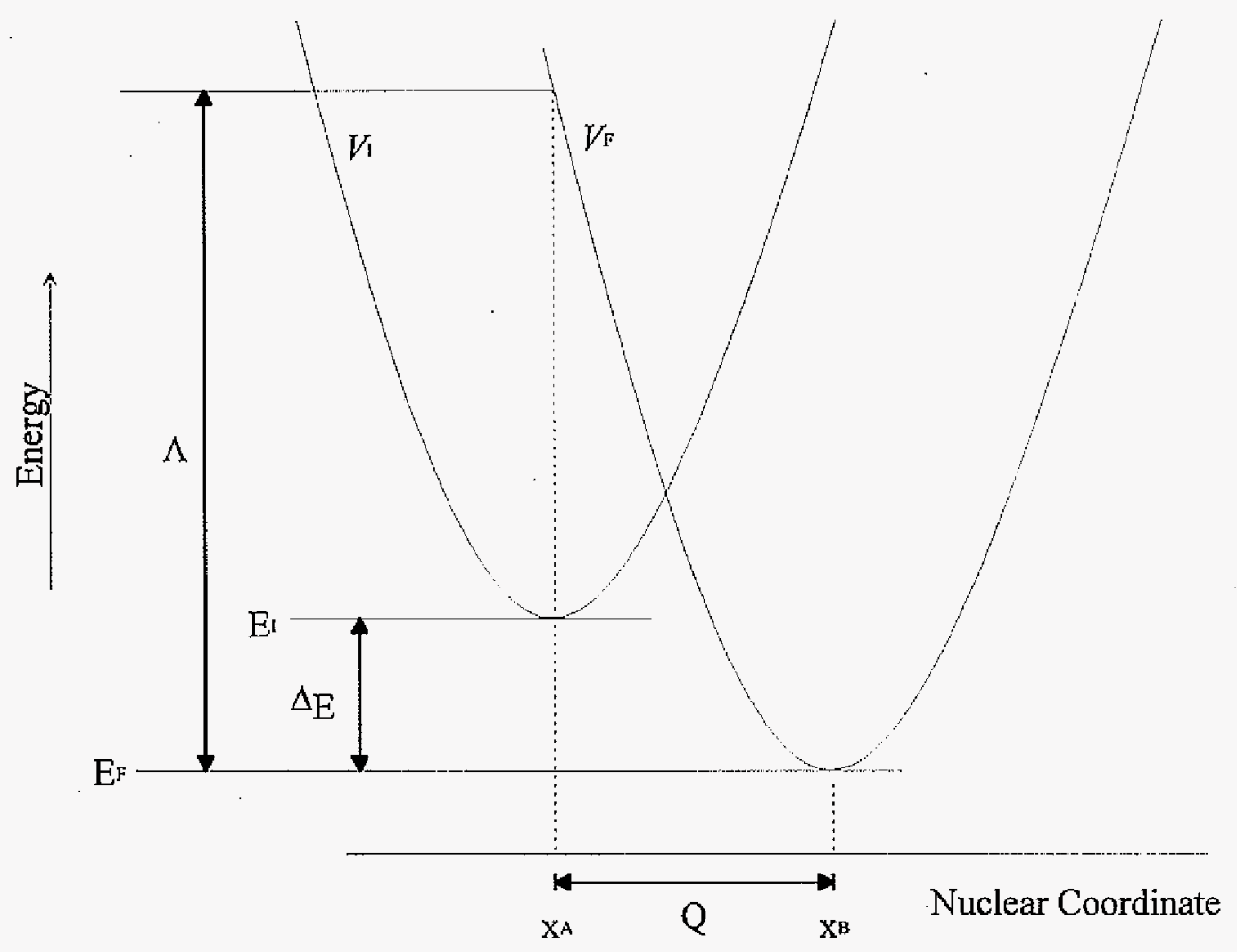

Figure 2.5 Scheme of the nuclear motions for a non-adiabatic energy transfer. Nuclear potential curves $V_{1}$ and $V_{\mathrm{F}}$ correspond to donor and acceptor states, respectively. Note that the reorganization energy, $\Lambda$, is required to displace the coordinate of the system from $\mathrm{x}_{\mathrm{A}}$ to $\mathrm{x}_{\mathrm{B}}$. See text for more discussion. 


$$
C^{2}(0, p)=\frac{S^{p} e^{-S}}{p !}
$$

Since $F(n=0, T \rightarrow 0 \mathrm{~K})=1$ in the low temperature limit, then substitution of Eq. (15) into Eq. (13) yields

$$
\mathrm{FC}=\frac{S^{p} e^{-s}}{p !}
$$

As indicated by Eq. (16), the Franck-Condon factors for phonons (intermolecular modes) follow a simple Poisson distribution formula. (Note that Eq.(16) also applies to intramolecular harmonic vibrations.) The Franck-Condon factor for a collection of oscillators has been determined [43,44] and is given below [45]. For a single oscillator,

$$
\mathrm{FC}=\frac{1}{2 \pi \hbar} \int_{-\infty}^{\infty} \exp \left[-S(2 \overline{\mathrm{n}}+1)-(\overline{\mathrm{n}}+1) e^{j \omega t}-\overline{\mathrm{n}} e^{-j \omega t}-i \Delta E t / \hbar\right] d t
$$

where

$$
\overline{\mathrm{n}}=\frac{1}{\exp (\hbar \omega / k T)-1}
$$

$\overline{\mathrm{n}}$ is the average vibrational quantum number at thermal equilibrium. By a Taylor series expansion of $e^{ \pm_{i}\left(\omega_{t} t\right.}$ terms, the general formula for the Franck-Condon factor for multiple oscillators is given as $[4,46]$

$$
\mathrm{FC}=\frac{1}{2 \pi \hbar} \int_{-\infty}^{\infty} f(t) e^{-i N: t / t} d t
$$

where

$$
\begin{gathered}
f(t)=\exp \left[G_{+}(t)+G_{-}(t)-\left(G_{+}(0)+G_{-}(0)\right)\right], \\
G_{+}(t)=\sum_{j} S_{j}\left(\overline{\mathrm{n}}_{j}+1\right) \exp \left(+i \omega_{j} t\right)
\end{gathered}
$$


and

$$
G_{-}(t)=\sum_{j} S_{j} \bar{n}_{j} \exp \left(-i \omega_{j} t\right)
$$

Note that $G_{+}(t)$ and $\mathrm{G}_{-}(t)$ represent absorption and emission of vibrational quanta at time $t$, respectively. $j$ indicates the modes in the multiple oscillator system. By combining Eq. (19), (20), (21) and (22) with integration over $t$, the Franck-Condon factor for arbitrary temperature has been derived $[5,46]$ as

$$
\mathrm{FC}=e^{-\sum_{j} S_{j}\left(2 \overline{\mathrm{n}}_{j} \div 1\right)} \prod_{j} \sum_{p}^{\infty} \sum_{p^{\prime}=0}^{p} \frac{\left[S_{j}\left(\overline{\mathrm{n}}_{j}+1\right)\right]^{p-p^{\prime}}\left[S_{j} \overline{\mathrm{n}}_{j}\right]^{p^{\prime}}}{\left(p-p^{\prime}\right) ! p^{\prime} !} \delta\left(-\frac{\Delta E}{\hbar}+\sum_{j} \omega_{j}\left(p-2 p^{\prime}\right)\right)
$$

where $j$ describes here the discrete intramolecular modes. Detailed derivation of Eq. (23) is discussed in Refs. [4,46].

\subsubsection{Single-site Absorption Profile and Absorption Spectrum}

For this thesis study, experimental hole spectra were used to determine the energy levels. To that end, the single site absorption profile must be known. Original hole spectra formulations (using different approach) were performed in Refs. [10,46]. In this section, the derivation of the single site absorption profile as well as the absorption spectrum will be discussed.

Based on the Fermi golden rule, the absorption spectrum is given by [47]

$$
\sigma(\Omega)=\frac{4 \pi^{2} \Omega}{\hbar c} \sum_{n} \sum_{n^{\prime}}\left|\left\langle j, n^{\prime}|\mu| i, n\right)\right|^{2} \delta(\Omega-\Delta E / \hbar)
$$

for an absorption from the initial electronic state $i$ to the final state $j$ ( $n$ and $n^{\prime}$ correspond to the vibrational levels of the initial and final state, respectively), where $\Delta E=E_{j, n^{\prime}}-E_{i, n}$ and $\mu$ represents the transition dipole moment operator. In the Condon approximation, 


$$
\left|\left\langle j, n^{\prime}|\mu| i, n\right\rangle\right|^{2}=|\langle j|\mu| i\rangle|^{2}\left|\left\langle n^{\prime} \mid n\right\rangle\right|^{2}=|\langle j|\mu| i\rangle|^{2} C^{2}\left(n, n^{\prime}\right)
$$

Eq. (24) is then $[5,46]$

$$
\sigma(\Omega)=\frac{4 \pi^{2} \Omega}{\hbar c} \sum_{n} \sum_{n^{\prime}}|\langle j|\mu| i\rangle|^{2} C^{2}\left(n, n^{\prime}\right) \delta_{n^{\prime}, \lambda}(\Omega-\Delta E / \hbar)
$$

As discussed in Section 2.3.1, in the low temperature limit $(T \rightarrow 0 \mathrm{~K}), n \rightarrow 0$ and $n^{\prime}=n+p$ $\rightarrow p$. Therefore, the Franck-Condon factor of the transition converges into simple Poisson formula (see Eq. (16)), which leads to

$$
\sigma(\Omega)=\frac{4 \pi^{2} \Omega}{\hbar c}|\langle j|\mu| i\rangle|^{2} \sum_{p=0}^{\infty} \frac{S^{p} e^{-S}}{p !} \delta_{p}(\Omega-\Delta E / \hbar) .
$$

By replacing the delta function by a line shape function $(l)$ and defining $v=\Delta E / \hbar$ as the center of the Lorentzian, the single-mode absorption spectrum is

$$
\sigma(\Omega-v)=\frac{4 \pi^{2}}{\hbar c}|\langle j|\mu| i\rangle|^{2} \sum_{p=0}^{\infty} \frac{S^{p} e^{-S}}{p !} l_{p}\left(\Omega-v-p \omega_{m}\right)
$$

where $\omega_{m}$ is the mean phonon frequency and the $p$-phonon transition is centered at $v+p \omega_{m}$ (the one-phonon transition is centered at $v+\omega_{m}$ and the zero-phonon transition at $v$ ). The $p$ phonon lineshape profile, $l_{p}$, is the result of convolution of one-phonon profile $\left(l_{l}\right) p$-times with itself. The absorption spectrum can be written as

$$
\sigma(\Omega-v) \equiv \sigma_{\text {int }} L(\Omega-v)
$$

with $L(\Omega)$ is normalized to unity. $\sigma_{\text {int }}$ is the integral absorption cross-section and $L(\Omega)$ is the single site absorption profile. Thus, in the low temperature limit $[10,47]$,

$$
L(\Omega-v)=\sum_{p=0}^{\infty} \frac{S^{p} e^{-S}}{p !} l_{p}\left(\Omega-v-p \omega_{m}\right)
$$


Several studies have extended it for the multi-mode single site profile $[4,5,48$ and references therein] as

$$
L(\Omega-v)=\prod_{j=1}^{N} \sum_{p=0}^{\infty} \frac{e^{-S_{j}} S_{j}{ }^{p}}{p !} l_{p}\left(\Omega-v-p \omega_{j}\right),
$$

where $j$ corresponds to the discrete phonon modes.

Because the absorption spectrum for a distribution of absorbing centers can be written as an integral of the single-site absorption profiles over the distribution of ZPL frequencies,

$$
A_{0}(\Omega)=\int d v N_{0}\left(v-v_{m}\right) L(\Omega-v),
$$

where subscript 0 indicates the time 0 , i.e., pre-burn absorption. $N_{0}\left(v-v_{m}\right)$ stands for the Gaussian distribution of the ZPL frequencies (often referred as a site distribution function, SDF), centered at $v_{m}$. Substitution of the single site profile in Eq. (32) leads to

$$
A_{0}(\Omega)=\prod_{j=1}^{N} \sum_{p=0}^{\infty} \frac{e^{-S_{j}} S_{j}^{p}}{p !} \int d v N_{0}\left(v-v_{m}\right) l_{p}\left(\Omega-v-p \omega_{j}\right) .
$$

The site distribution function after a burn (time $\tau$ ) is then defined [10] as

$$
N_{\tau}\left(v-v_{m}\right)=N_{0}\left(v-v_{m}\right) e^{-P \phi \alpha r l\left(\omega_{\mathrm{B}}-v\right)},
$$

where $P, \phi$ and $\sigma$ are photon flux, hole-burning quantum yield and optical cross section parameters, respectively. Then, the absorption spectrum following hole-burning at a frequency $\omega_{\mathrm{B}}$ for a time $\tau$ can be written as

$$
\begin{gathered}
A \tau(\Omega)=\int d v N_{\tau}\left(v-v_{m}\right) L(\Omega-v) \\
=\prod_{j=1}^{N} \sum_{p=0}^{\infty} \frac{e^{-S_{j}} S_{j}^{p}}{p !} \int d v N_{0}\left(v-v_{m}\right) e^{-p_{\phi} \phi L_{L} /\left(\omega_{\mathrm{B}}-v\right)} l_{p}\left(\Omega-v-p \omega_{j}\right)
\end{gathered}
$$


Note that Eq. (35) neglects the photoproduct absorption. A detailed investigation of hole shape and the influence of the photoproduct is given in Ref. [23]. For arbitrary temperatures, the FC factor needs be substituted by Eq. (23). As in the derivation of Eq. (28), the delta function is replaced by a line shape function and, by following the same procedure as above, the single site absorption profile for the arbitrary temperature results in

$$
L(\Omega-v)=e^{-\sum_{j} S_{j}\left(2 \overline{\mathrm{n}}_{\left.j^{\prime}+1\right)}\right.} \prod_{j} \sum_{p}^{\infty} \sum_{p^{\prime}=0}^{p} \frac{\left[S_{j}\left(\overline{\mathrm{n}}_{j}+1\right)\right]^{p-p^{\prime}}\left[S_{j} \overline{\mathrm{n}}_{j}\right]^{p^{\prime}}}{\left(p-p^{\prime}\right) ! p^{\prime} !} l_{p, p^{\prime}}\left(\Omega-v-\omega_{j}\left(p-2 p^{\prime}\right)\right) .
$$

Then, the absorption spectrum for the arbitrary temperature is given by

$$
\begin{aligned}
& A_{\mathrm{t}}(\Omega)=e^{-\sum_{j} S_{j}\left(2 \overline{\mathrm{n}}_{j}+1\right)} \prod_{j} \sum_{p}^{\infty} \sum_{p^{\prime}=0}^{p} \frac{\left[S_{j}\left(\overline{\mathrm{n}}_{j}+1\right)\right]^{p-p^{\prime}}\left[S_{j} \overline{\mathrm{n}}_{j}\right]^{p^{\prime}}}{\left(p-p^{\prime}\right) ! p^{\prime} !} . \\
& \times \int d v N_{0}\left(v-v_{m}\right) e^{-p \phi \sigma \tau L\left(\omega_{\mathrm{B}}-v\right)} l_{p, p^{\prime}}\left(\Omega-v-\omega_{j}\left(p-2 p^{\prime}\right)\right)
\end{aligned}
$$

This formula was utilized for the simulations in Ref. [49] and Chapter 3. Note that in the hole profile simulation program (see Appendix), an asymmetric phonon profile $\left(l_{p, p}\right)$ is employed which has a Gaussian shape on the low-energy side and a Lorentzian shape on the higherenergy side. The hole spectrum is the difference between the after-burn absorption spectrum at time $\tau, A_{\tau}(\Omega)$, and pre-burn spectrum, $A_{0}(\Omega)$.

\subsubsection{Exciton Level Structures}

Researchers interested in the excited electronic states of photosynthetic antenna complexes have recently utilized exciton theory to calculate band structures. The following is a review of a Frenkel exciton theory, first within the static lattice approximation, i.e. the exciton-phonon coupling is neglected, as well as the effects of energy disorder. For detailed 
discussion on the effects of energy disorder (diagonal or off-diagonal), the reader can consult Ref. $[14,51,52]$.

Energy states of an isolated molecule are described with the eigenfunctions $\varphi^{i}$ and the energies $E^{i}$ :

$$
H \varphi^{i}=E^{i} \varphi^{i}
$$

where $i$ corresponds to the ground state $(g)$ or excited state $(e)$ of the molecule. For a dimer of identical molecules with the monomers interacting through the intermolecular potential $V$, the total energy for the system in the ground state can be written as

$$
\left\langle\psi^{g}\left|H_{1}+H_{2}+V\right| \psi^{g}\right\rangle=2 E^{g}+\mathrm{V}_{00}
$$

where

$$
\psi^{g}=\varphi_{1}^{g} \varphi_{2}^{g}
$$

and $V_{00}$ is van der Waals interaction (or dispersion energy) for the ground state. In contrast, the wavefunction for the excited dimer can be described as

$$
\Psi_{ \pm}=c_{1}^{e} \psi_{1}^{e}+c_{2}^{e} \psi_{2}^{e}=c_{1}^{e} \varphi_{1}^{e} \varphi_{2}^{g}+c_{2}^{e} \varphi_{1}^{g} \varphi_{2}^{e}
$$

where coefficients $c_{1}$ and $c_{2}$ are normalized and orthogonal. Therefore, the energies of the excited dimer states are then given by

$$
\left\langle\Psi_{ \pm}\left|H_{1}+H_{2}+V\right| \Psi_{ \pm}\right\rangle=E^{g}+E^{e}+\mathrm{V}_{11} \pm \mathrm{V}_{12}
$$

where $V_{11}$ is dispersion energy for the excited dimer and $V_{12}$ is resonance energy integral:

$$
\begin{aligned}
& \mathrm{V}_{11} \equiv\left\langle\psi_{1}^{e}|V| \psi_{1}^{e}\right\rangle=\left\langle\psi_{2}^{e}|V| \psi_{2}^{e}\right\rangle \equiv \mathrm{V}_{22} \\
& \mathrm{~V}_{12} \equiv\left\langle\psi_{1}^{e}|V| \psi_{2}^{e}\right\rangle=\left\langle\psi_{2}^{e}|V| \psi_{1}^{e}\right\rangle \equiv \mathrm{V}_{21}
\end{aligned}
$$

The dimer levels are split by $2 \mathrm{~V}_{12}$ (Davydov splitting or exciton splitting) [53]. Also, the average energy of these two levels has been shifted with respect to the ground state by an 
amount of $\left(\mathrm{V}_{11}-\mathrm{V}_{00}\right)$, which is sometimes referred to as displacement energy and usually leads to a red shift of the absorption band. For discussion of inequivalent site energies, consult Section 3.1 in Chapter 2 of Ref. [54]. Note that selective excitation of either of the spectrally well-resolved dimer state $\left(\Psi_{ \pm}\right)$is necessary for creation of delocalized dimer states. The excitation eventually needs to be localized on one of the two monomers for excitation energy transfer to occur.

The excitons in molecular crystal (or aggregate) can easily be described by extending the above treatment. For a system consisting of $n$ molecules, in analogous to dimer system, the wavefunction for excitation energy localized on molecule $\alpha$ is given by

$$
\psi_{\alpha}^{e}=\varphi_{\alpha}^{e} \prod_{\beta \neq \alpha, \beta=0}^{n-1} \varphi_{\beta}^{g}
$$

The delocalized aggregate states are linear combinations of the $\psi_{a}^{e}$ 's. The Hamiltonian for the exciton states is

$$
H=\sum_{\alpha} H_{\alpha}+\frac{1}{2} \sum_{\alpha, \beta} V_{\alpha \beta}
$$

where $V_{\alpha \beta}$ is the interaction between molecules $\alpha$ and $\beta$ and $H_{\alpha}$ is the Hamiltonian of the $\alpha^{\text {th }}$ molecule which is defined by

$$
H_{\alpha} \varphi_{\alpha}^{e}=\varepsilon^{e} \varphi_{\alpha}^{e}
$$

For general solution to Eq. (45) and more details, see Ref. [53]. The Hamiltonian for a linear aggregate has been diagonalized and the expressions for the exciton wavefunctions can be found in Ref. [55]. The excited energy levels are split by $\sim 4 \mathrm{~V}_{\alpha \beta}$ for linear or circular aggregates. 
In order to consider exciton-phonon coupling, molecular displacements from equilibrium positions need to be considered. One starts with the excitonic Hamiltonian Ref. [53]

$$
H_{e x}(\underline{R})=\sum_{n}\left[\left(E+\sum_{m}^{\prime} D_{n u l}(\underline{R})\right) B_{n}^{+} B_{n}+\sum_{m}^{\prime} M_{m m}(\underline{R}) B_{m}^{+} B_{n}\right]
$$

where matrix element $D$ describes the interaction between the $n^{\text {th }}$ excited molecule and all other ground state molecules and $M$ is responsible for excitation transfer from the $n^{\text {th }}$ molecule to the $m^{\text {th }}$ molecule (resonance-energy transfer). $E$ is the excitation energy of an isolated molecule and the operators $B_{n}{ }^{+}$and $B_{n}$ are the creation and annihilation operators for the $n^{\text {th }}$ site. As seen above, $H_{e x}$ is dependent on the lattice configuration, $\underline{R}$, which is introduced by the matrix elements $D$ and $M$. Expanding by a power series, to displace $\underline{R}$, relative to the equilibrium position of the molecules $(R=0)$ leads to

$$
H_{e x}(\underline{R})=H_{e x}(0)+H_{e x-p h}^{(1)}+H_{e x-p h}^{(2)},
$$

where non-linear terms are neglected. The Hamiltonians for linear exciton-phonon coupling are given by

$$
H_{e x-p h}^{(1)}=\sum_{n} \sum_{m} B_{m}^{+} B_{n} \sum_{\alpha}\left[R_{n}^{\alpha}\left(\frac{\partial M_{m m}}{\partial R_{n}^{\alpha}}\right)_{0}+R_{m}^{\alpha}\left(\frac{\partial M_{m m}}{\partial R_{m}^{\alpha}}\right)_{0}\right]
$$

and

$$
H_{e x-p h}^{(2)}=\sum_{n} \sum_{m} B_{n}^{+} B_{n} \sum_{\alpha}\left[R_{n}^{\alpha}\left(\frac{\partial D_{n m}}{\partial R_{n}^{\alpha}}\right)_{0}+R_{m}^{\alpha}\left(\frac{\partial D_{m m}}{\partial R_{m}^{\alpha}}\right)_{0}\right],
$$

where $\alpha$ denotes the six degrees of freedom of the molecules ( 3 for translation and 3 for rotation). The subscript 0 designates evaluation at the ground state configuration. $M$ 
represents the resonance-energy transfer matrix element between two molecules and, thus, $H^{(1)}$ is responsible for the elastic and inelastic scattering of the excitons by phonons [14].

On the other hand, $H^{(2)}$ is associated with the dispersion term $D . H^{(2)}$ describes lattice distortion produced by electronic excitation. Since the excited and ground state molecules have different forces with their neighbors, excitation often causes displacement of molecules to new equilibrium positions. From Eqs. (47), (48) and (50), the linear term of the matrix element $D$, which describes the distortion, can be written as

$$
D_{m m}(1)=\sum_{\alpha}\left[R_{n}^{\alpha}\left(\frac{\partial D_{n m}}{\partial R_{n}^{\alpha}}\right)_{0}+R_{m}^{\alpha}\left(\frac{\partial D_{m m}}{\partial R_{m}^{\alpha}}\right)_{0}\right] .
$$

For a dimer system, $D$ and $A$,

$$
D(1)=\sum_{\alpha}\left[R_{D}^{\alpha}\left(\frac{\partial D_{D A}}{\partial R_{D}^{\alpha}}\right)_{0}+R_{A}^{\alpha}\left(\frac{\partial D_{D A}}{\partial R_{A}^{\alpha}}\right)_{0}\right]
$$

If one considers a dimer system, the displacement of molecules can be expressed in terms of ground-state phonon coordinates [50]:

$$
D(1)=\sum_{p} Q_{p}\left(\frac{\partial D_{D A}}{\partial Q_{p}}\right)_{0},
$$

where $Q_{p}$ is the phonon coordinate for mode $p$ and it can be conveniently expressed in terms of creation and annihilation operators [50] as

$$
Q_{p}=\left(\frac{\hbar}{2 \omega_{p}}\right)^{1 / 2}\left(b+b^{+}\right)
$$

Derivations and further treatment of the energy transfer matrix element have been discussed in Ref. [50,53]. Also, for discussion about inter-exciton level relaxation process, the reader can refer to Ref. [54]. 
Hamiltonian models for exciton levels of $\mathrm{C}_{n}$ cyclic (ringed) choromophore arrays, which were utilized for simulations in Chapter 4, were developed in Refs. [18,52]. The Hamiltonian in the absence of disorder was given [18] as

$$
H_{O}=e \sum_{\mathrm{a}=0}^{n-1}|\alpha\rangle\left\langle\alpha\left|+\sum_{\alpha, \beta=0}^{n-1} V_{\alpha, \beta}\right| \alpha\right\rangle\langle\beta|,
$$

where $e$ is the excitation energy of chromophore and $\alpha$ and $\beta$ denote its sites. Then, in the nearest dimer-dimer coupling approximation, the exciton level energies were derived [18] as

$$
E_{i}^{i}=e_{i}+2 V_{I} \cos (2 \pi j / n)
$$

for lower manifold and

$$
E_{u}^{j}=e_{u}+2 V_{u} \cos (2 \pi j / n)
$$

for upper manifold, where $j=0,1, \ldots, n-1$ with $\mathrm{n}$ the number of dimers in the ring, $e_{l}$ and $e_{u}$ are the energies of the two levels of the basic dimer and $V_{l}$ and $V_{u}$ are the nearest neighbor dimer-dimer coupling energies for the lower and upper manifolds, respectively. The coupling between the respective exciton levels of two manifolds was obtained as

$$
H_{u l}^{j}=2 V_{u l} \cos (2 \pi j / n) .
$$

The dimer-dimer couplings $V_{l}, V_{u}$ and $V_{u l}$ can be determined using the monomer-monomer coupling energies [51]. The Hamiltonian in the presence of disorder can be written [51] as

$$
H=H_{0}+H_{\lambda}+H_{\mathrm{v}},
$$

where $H_{\lambda}$ and $H_{\mathrm{v}}$ govern the diagonal and off-diagonal disorder, respectively, and are defined by

$$
H_{\lambda}=\sum_{\alpha} \lambda_{\alpha}|\alpha\rangle\langle\alpha|
$$

and 


$$
H_{\mathrm{v}}=\sum_{\alpha} \mathrm{v}_{\mathrm{a}}(|\alpha\rangle\langle\alpha+1|+| \alpha+1\rangle\langle\alpha|)
$$

where $\lambda_{\alpha}$ is the diagonal energy defect at site $\alpha$ and $\mathrm{v}_{\alpha}$ is the defect associated with coupling between sites $\alpha$ and $\alpha+1$. For $H_{\lambda}$, the coupling between the delocalized levels $r$ and $s$ is given by

$$
\left\langle r\left|H_{\lambda}\right| s\right\rangle=\frac{1}{n} \sum_{a} \lambda_{\alpha} B^{(r-s) a},
$$

where

$$
B=e^{i 2 \pi / n} .
$$

The energy defect patterns can be calculated using superpositions of basic defect patterns (BDP). The BDP for a $\mathrm{C}_{9}$-array are given in Ref. [51]. For further discussion of symmetryadapted BDP for analysis of energy disorder effects on cyclic excitonic systems see Appendix of Ref. [14].

\section{References:}

1. Fridrich, J, and Haarer, D. Angew. Chem. Int. Ed. Engl. 1984, 23, 113.

2. Milanovich, N. Ph.D. Dissertation, Iowa State University, 1999.

3. Völker, S. in Relaxation Processes in Molecular Excited States (Fünfschilling, J ed.), Kluwer, Dordrecht, 1989, p. 113.

4. Lyle, P. A. Ph.D. Dissertation, Iowa State University, 1993.

5. Lyle, P. A., Kolaczkowski, S. V. and Small, G. J. J. Phys. Chem. 1993, 97, 6926.

6. Jankowiak, R., Hayes, J. M. and Small, G. J. Chem. Rev. 1993, 93, 1471.

7. Jankowiak, R. and Small G. J. in The Photosynthetic Reaction Center (Vol.2, Deisenhofer, J. and Norris, J. eds.), 1993, Academic Press, New York, p. 133. 
8. Reddy, N. R. S., Lyle, P. A. and Small, G. J. Photosynth. Res. 1992, 31, 167.

9. Jankowiak, R. and Small, G. J. Science $1987,237,618$.

10. Hayes, J. M., Gillie, J. K., Tang, D. and Small, G. J. Biochim. Biophys. Acta 1988, $932,287$.

11. Pieper, J., Voigt, J, Renger, G. and Small, G. J. Chem. Phys. Lett. 1999, 310, 296.

12. Reddy, N. R. S., Picorel, R. and Small, G. J. J. Phys. Chem. 1992, 96, 6458.

13. Reddy, N. R. S., Cogdell, R. J., Zhao, L. and Small, G. J. Phtochem. Photobiol. 1993, 57,35 .

14. Wu, H.-M. Ph.D. Dissertation, Iowa State University, 1998.

15. Wu, H.-M., Ratsep, M., Young, C. S., Jankowiak, R., Blankenship, R. E., Small, G. J. Biophys. $J_{2000}, 79,1561$.

16. Pieper, J., Ratsep, M., Jankowiak, R., Irrgang, K.-D., Voigt, J., Renger, G., Small, G. J. J. Phys. Chem. A 1999, 103, 2412.

17. Wu, H.-M., Ratsep, M., Jankowiak, R., Cogdell, R. J., Small, G. J. J. Phys. Chem. B 1998, 102, 4023 .

18. Ratsep, M., Wu, H.-M., Hayes, J. M., Small, G. J. Spectrochim. Acta, Part A 1998, $54 \mathrm{~A}, 1279$.

19. Gorokhovskii, A. A., Kaarli, R. K. and Rebane, L. A. JETP Lett. 1974, 20, 216.

20. Kharlamov, B. M., Personov, R. I., and Bykovskaya, L. A. Opt. Commun. 1974, 12, 191.

21. Hayes, J. M. and Small, G. J. Chem. Phys. 1978, 27, 151.

22. Hayes, J. M., Jankowiak, R. and Small, G. J. in Persistent Spectral Hole-Burning:

Science and Applications (Moerner, W. E. ed.), 1988, Springer-Verlag, New York, p. 153.

23. Reinot, T. and Small, G. J. Chem. Phys., 2001, 114, 9150.

24. Hayes, J. M., Stout, R. P. and Small, G. J. J. Phys. Chem. 1981, 74, 4266.

25. Shu, L. and Small, G. J. Chemical Physics 1990, 141, 447. 
26. Kenney, M. J., Jankowiak, R. and Small, G. J. Chemical Physics 1990, 146, 47.

27. Reinot, T. and Small, G. J. J. Chem. Phys. 2000, 113, 10207.

28. Shu, L. and Small, G. J. J. Opt. Soc. Am B 1992, 9, 733.

29. Reinot, T., Hayes, J. M. and Small, G. J. Chem. Phys., 1999, 110, 4820.

30. Jackson, B. and Silbey, R. J. Chem. Phys. Lett. 1983, 99, 331.

31. Silbey, R. J. and Kassner, K. J. Lumin. 1987, 36, 283.

32. Jankowiak, R. and Small, G. J. Chem. Phys. Lett. 1993, 207, 436.

33. Koedijk, J. M. A, Wannemacher, R., Silbey, R. J. and Völker, S. J. Phys. Chem. 1996, 100, 19945.

34. den Hartog, F. T. H., van Papendrecht, C., Silbey, R. J. and Völker, S. J. Chem. Phys. $1999,110,1010$.

35. Reinot, T., Zazubovich, V., Hayes, J. M. and Small, G. J. J. Phys. Chem. B 2001, 105, 5083.

36. Marcus, R. A. J. Chem. Phys. 1956, 24, 966.

37. Marcus, R. A. J. Chem. Phys. 1965, 43, 679.

38. Marcus, R. A. and Sutin, N. Biochim. Biophys. Acta 1985, 811, 265.

39. Jortner, J. J. Chem. Phys. 1976, 64, 4860.

40. Hopfield, J. J. Proc. Natl. Acad. Sci. USA 1974, 71, 3640.

41. Atkins, P. W. in Molecular Quantum Mechanics, Oxford, London, 1983, p. 198.

42. Manneback, C. Physica Grav. 1951, 17, 1001.

43. Kubo, R. Phys. Rev. 1952, 86, 929.

44. Lax, M. J. Chem. Phys. 1952, 20, 1752.

45. DeValut, D. Quant. Rev. of Biophys. 1980, 13, 387.

46. Hayes, J. M., Lyle, P. A. and Small, G. J. J. Phys. Chem. 1994, 98, 7337. 
47. Pryce, M. H. L. in Phonons in Perfect Lattice and in Lattices with Point Imperfections (Stevenson, R. W. H. ed.), 1966, Plenum Press, New York, p.403.

48. DeVault, D. Quantum-Mechanical Tunneling in Biological Systems ( $2^{\text {nd }}$ ed.), 1984, Cambridge University Press, New York.

49. Hayes, J. M., Matsuzaki, S., Rätsep, M. and Small, G. J. J. Phys. Chem. B 2000, 104, 5625 .

50. Johnson, C. K. and Small, G. J. in Excited States (Vol.6, Lim, E. C. ed.) 1982, Academic Press, New York, p. 97.

51. Wu, H-. M. and Small, G. J. Chem. Phys. 1997, 218, 225.

52. Wu, H.. M. and Small, G. J. J. Phys. Chem. B 1998, 102, 888.

53. Davydov, A. S. in Theory of Molecular Excitons 1971, Plenum, New York.

54. van Amerongen, H., Valkunas, L. and van Grondelle, R. Photosynthetic Excitons 2000, World Scientific, Singapore.

55. Fidder, H., Knoester, J. and Wiersma, D. A. J. Chem. Phys. 1991, 95, 7880. 


\title{
CHAPTER 3. ENERGY TRANSFER KINETICS AND LOW ENERGY VIBRATIONAL STRUCTURE OF THE THREE LOWEST ENERGY QY-STATES OF THE FENNA-MATTHEWS-OLSON ANTENNA COMPLEX
}

A paper published in the Journal of Physical Chemistry B 2000, 104, 9564.

S. Matsuzaki, V. Zazubovich, M. Rätsep, J. M. Hayes, and G. J. Small

\begin{abstract}
Burn wavelength $\left(\lambda_{\mathrm{B}}\right)$-dependent nonphotochemical hole spectra are reported for the lowest energy Qy-absorption band of the Fenna-Matthews-Olson (FMO) trimer complex from Prosthecochloris aestuarii. This band at $825 \mathrm{~nm}$ is contributed to by three states that stem from the lowest energy state of the subunit of the trimer. The spectra reveal unusually rich and quite sharp low energy satellite structure that consists of holes at $18,24,36,48,72$, 120 and $165 \mathrm{~cm}^{-1}$ as measured relative to the resonant hole at $\lambda_{\mathrm{B}}$. The possibility that some of these holes are due to correlated downward energy transfer from the two higher energy states that contribute to the $825 \mathrm{~nm}$ band could be rejected. Thus, the FMO complex is yet another example of a photosynthetic complex for which structural heterogeneity results in distributions for the values of the energy gaps between $Q_{y}$-states. The results of theoretical simulations of the hole spectra are consistent with the above holes being due to intermolecular phonons and low energy intramolecular vibrations of the bacteriochlorophyll $a(\mathrm{BChl} a)$ molecule. The $36 \mathrm{~cm}^{-1}$ and higher energy modes are most likely due to the intramolecular BChl $a$ modes. The simulations lead to the determination of the Huang-Rhys (S) factor for all modes. They range between 0.05 and 0.25 in value. The temperature
\end{abstract}


dependencies of the spectral dynamics for the three contributing states are similar to those reported for the FMO complex from Chlorobium tepidum (Rätsep et al., J. Phys. Chem. B 2000, 103, 5736). The contribution to the dynamics from pure dephasing/spectral diffusion due to the glass-like two-level systems of the protein is identical for all three states. The lifetimes of the highest and intermediate energy $Q_{y}$-states due to downward energy transfer are 26 and $99 \mathrm{ps,}$, respectively, at liquid helium temperatures.

\section{Introduction}

The Fenna-Matthews-Olson (FMO) bacteriochlorophyll $a(\mathrm{BChl} a)$ antenna complex of the green sulfur bacterium Prosthecochloris aestuarii was the first photosynthetic complex whose structure was determined by $\mathrm{X}$-ray diffraction [1]. The structure led to many studies of its $Q_{y}\left(S_{1}\right)$-electronic structure and excitation energy transfer $(E E T)$ dynamics (for reviews see Refs. [2,3]). The complex is a $\mathrm{C}_{3}$ trimer of subunits, each containing seven symmetry inequivalent $\mathrm{BChl} a$ molecules. Nearest neighbor $\mathrm{Mg}$... Mg distances within the subunit are $\approx 11-14 \AA$ with the largest pairwise excitonic couplings in the $\approx 50-200 \mathrm{~cm}^{-1}$ range (calculated using point monopoles with a dielectric constant, $\varepsilon$, of 1 [4]). The largest coupling between BChl a molecules belonging to different subunits is $\approx 15 \mathrm{~cm}^{-1}$. Recently, the X-ray structure of the FMO complex from Chlorobium tepidum was determined [5]. The relative orientations of the $\mathrm{BChl} a$ molecules and the distances between them are very similar to those of $P$. aestuarii although there are some potentially significant differences in $\mathrm{H}$ bonding and $\mathrm{BChl} a$-residue distances that may contribute to the differences between the $4.2 \mathrm{~K} \mathrm{Q}_{\mathrm{y}}$-absorption spectra of $P$. aestuarii and $C b$. tepidum seen in Figure 1. 
Pearlstein and co-workers were the first to perform excitonic calculations on the FMO complex of $P$. aestuarii. In the end, Pearlstein concluded that it is necessary to include the full trimer, rather than just the subunit, to best explain the low temperature absorption and CD spectra [4]. Consideration of the trimer was stimulated by the $4.2 \mathrm{~K}$ hole burning results of Johnson and Small [6] which showed that the $825 \mathrm{~nm}$ absorption band (see Figure 1) is contributed to by at least two states. van Mourik et al. [7] reached the same conclusion based on polarized triplet-singlet difference spectra. Concerning the states of the subunit, Pearlstein found that they are mini-excitons with excitation primarily on 2-3 $\mathrm{BChl} a$ molecules. The mini-exciton picture also emerged from the calculations of Gülen [8] and Louwe et al. [9] There is general agreement that the $825 \mathrm{~nm}$ absorption band is due to three trimer states which stem from the lowest energy $Q_{y}$-state of the subunit that is mainly localized on a single $\mathrm{BChl} a$ molecule. This molecule is most likely $\mathrm{BChl} 3$ [9] or 6 [8] according to the numbering scheme of Ref. [1]. For perfect $\mathrm{C}_{3}$ symmetry, there is a doubly degenerate $\mathrm{E}$ state and non-degenerate $\mathrm{A}$ state that are polarized perpendicular and parallel to the $\mathrm{C}_{3}$-axis, respectively. Energy disorder due to structural heterogeneity results in removal of the degeneracy of the E state and mixing of the A and E states. Based on the results of Gülen [8] and Louwe et al. [9], it is most likely that the A state is weakly absorbing relative to the $\mathrm{E}$ state in the absence of energy disorder. However, which of these two states lies lowest in energy is an unsettled question.

Further evidence for the $825 \mathrm{~nm}$ band being contributed to by three states came from the low temperature accumulated photon echo experiments of Louwe and Aartsma [10] on the FMO complex of $P$. aestuarii and the non-photochemical hole burning experiments of Rätsep et al. [11] on the FMO complex of Cb. tepidum. Both works showed that the 
lifetimes of states excited at the high energy side and maximum of the $825 \mathrm{~nm}$ band are significantly shorter than that of the state excited at the low energy side which is $2 \mathrm{~ns}$, about the value expected for the lifetime of an isolated $\mathrm{BChl} a$ molecule. The hole burning data led to lifetime values of 117 and $37 \mathrm{ps}$ for $\lambda_{\mathrm{B}}$ (burn wavelength) equal to 825 and $823 \mathrm{~nm}$, respectively, which are similar to those reported by Louwe and Aartsma. Both groups concluded that these two lifetimes are determined by downward excitation energy transfer (EET). Based on fitting of the $825 \mathrm{~nm}$ absorption band of $C b$. tepidum, Rätsep et al. [11] suggested that it is contributed to by the absorption bands of three states located near 823 , 825 , and $827 \mathrm{~nm}$, with each band carrying a static inhomogeneous broadening of about $50 \mathrm{~cm}^{-1}$ and the same intensity. This suggests that one may be in the strong energy disorder limit. However, the results of van Mourik et al. [7] argue against this limit.

Presented here are non-photochemical hole burning (NPHB) data and spectra for the FMO complex of $P$. aestuarii. There were two main objectives of the experiments. The first was to elucidate the excitation energy transfer (EET) and spectral dynamics of the three trimer states that contribute to the $825 \mathrm{~nm}$ band and compare them with those of $\mathrm{Cb}$. tepidum. The results show that the kinetics for downward EET from the two higher energy states are similar in the two species as is the temperature dependence $(1.8-10 \mathrm{~K})$ of the spectral dynamics due to the two-level systems (TLS) of the protein. The second objective was to obtain more [12] detailed spectra that report on the unusual and rich low energy satellite hole structure observed when the burn wavelength $\left(\lambda_{B}\right)$ is tuned from the low to high energy side of the $825 \mathrm{~nm}$ band. The energies of the satellite holes, as measured relative to the resonant zero-phonon hole at $\lambda_{\mathrm{B}}$, are in the range 18 to $\approx 120 \mathrm{~cm}^{-1}$. The results lead to two possible interpretations for the satellite structure: that it is due to pseudo-phonon sideband holes, 
and/or to correlated downward EET from higher to lower energy states. It is emphasized that both interpretations are relevant to the interpretation of the EET kinetics. It is concluded that the first interpretation is correct and that the satellite structure is due to both intermolecular phonons and very low frequency intramolecular modes of $\mathrm{BChl} a$.

\section{Materials and Methods}

Samples of the isolated FMO complex from $P$. aestuarii were kindly provided by Professor T. Aartsma at Leiden University. The green sulfur bacterium was grown in a mixed culture as described in [13]. The protein was isolated and purified as described by Francke and Amesz [14]. Complexes were dissolved in $50 \mathrm{mM} \mathrm{HCl}$-Tris buffer at pH 8.3. Glycerol was added $(70 \% \mathrm{v} / \mathrm{v})$ to ensure good glass formation. Samples were contained in gelatin or plastic capsules with an optical pathlength of 5 and $8 \mathrm{~mm}$ respectively. The optical density of the sample at $825 \mathrm{~nm}$ was adjusted to $\approx 0.3$ at $4 \mathrm{~K}$. A Janis $20 \mathrm{DT}$ liquid helium cryostat was used for low temperature measurements. Temperatures were stabilized with a Lakeshore Cryotronics Model 330 temperature controller and measured with a silicon diode (accuracy $\pm 0.1 \mathrm{~K}$ ).

The laser used for hole burning was a Coherent CR899-29 Autoscan Ti:Sapphire laser pumped by a $15 \mathrm{~W}$ Coherent Innova 200 Ar-ion laser. Laser intensity was stabilized using an LS 100 power stabilizer (Cambridge Research and Instrumentation.) The burn intensities and times used are given in the Figure captions.

The hole spectra were obtained with the thick etalon of the above Ti:sapphire laser

removed which led to a linewidth of $0.07 \mathrm{~cm}^{-1}$. The spectra were recorded with a Bruker IFS $120 \mathrm{HR}$ Fourier transform (FT) spectrometer operated at a resolution of $1 \mathrm{~cm}^{-1}$. Hole spectra 
are the difference between the post-burn and pre-burn spectra. Most of the spectra reported are the average of 200 scans with an acquisition time of $8 \mathrm{~min}$. The intensity of the white light from the spectrometer at the sample was $\approx 2 \mathrm{~mW} / \mathrm{cm}^{2}$. A Glan-Thompson polarizer was used in the polarized hole burning experiments and placed before the sample, in-line with the spectrometer's white light beam. The vertically polarized laser beam was perpendicular to the optical axis. Post-burn spectra were recorded with horizontal polarization first and vertical polarization second. (Pre-burn absorption spectra were recorded with vertical and horizontal polarization of the probe beam in order to account for the possibility that the probe beam of the FT spectrometer is slightly polarized.) This order was chosen because of the spontaneous hole filling (SPHF) whose kinetics are dispersive; easier to burn sites are easier to fill [15]. It ensures that any polarization effects observed for the low energy satellite hole structure are real. This structure was observed to be uniformly and preferentially polarized parallel to the laser polarization.

For measurement of the dependence of the widths of zero-phonon holes on temperature the above Ti:sapphire laser was used with an actively stabilized linewidth $<20$ MHz. Spectra were recorded in the fluorescence excitation mode. Fluorescence was detected and processed by a GaAs photomultiplier tube (Hamamatsu) and photon counter (SR-400, Stanford Research Instruments). Rejection of scattered laser light was accomplished with two long wavelength-pass filters (RG 850, Coherent-Ealing) and a broad band interference filter (P60-940, Coherent-Ealing). This combination allowed for fluorescence detection between $\approx 910$ and $930 \mathrm{~nm}$. Laser intensities used for hole burning were in the range $20-400 \mu \mathrm{W} / \mathrm{cm}^{2}$, depending on the burn wavelength. Hole depths were 
typically $\approx 10 \%$. For hole reading, the laser was attenuated by a factor of $10-100$ in order to avoid hole burning during reading.

\section{Results and Discussion}

Some general features of the hole spectra. Persistent NPHB spectra for the FMO complex of $P$. aestuarii were obtained with burn wavelengths $\left(\lambda_{\mathrm{B}}\right)$ between 805.0 and $829.3 \mathrm{~nm}$. For each $\lambda_{\mathrm{B}}$-value spectra were recorded with several burn fluences. Figure 2 shows hole spectra obtained with $\lambda_{\mathrm{B}}=823 \mathrm{~nm}$, which is at the high energy side of the $825 \mathrm{~nm}$ band. For ease of discussion, the absorption spectrum is also shown. The sharpest and deepest zero-phonon hole $(\mathrm{ZPH})$ is coincident with $\lambda_{\mathrm{B}}$, referred to hereafter as the resonant hole. The satellite holes $\mathrm{a}, \mathrm{b}$ and $\mathrm{c}$ are part of the low energy satellite hole structure referred to in the Introduction. The feature indicated by the arrow is a pseudo-phonon sideband hole (PSBH) associated with the resonant hole, vide infra. It is displaced from the resonant hole by $18 \mathrm{~cm}^{-1}$. The broad satellite features at wavelengths shorter than $823 \mathrm{~nm}$ are very similar to those observed by Johnson and Small and, recently, by Franken et al [12]. The positive absorption to the left of the broad and relatively intense hole at $\approx 814 \mathrm{~nm}$ is its blue-shifted anti-hole (positive absorption) that is a signature for NPHB of $\mathrm{S}_{1} \pi \pi^{*}$ states $[16,17]$. This anti-hole is interfered with by the holes associated with 805 and $800 \mathrm{~nm}$ absorption bands. Johnson and Small favored the interpretation that has the high energy satellite holes being due to their associated $Q_{y}$-states being excitonically correlated with the state(s) directly burned at $\lambda_{\mathrm{B}}$. It is possible, however, that they are due, at least in part, to structural changes produced by NPHB of the state at $\lambda_{\mathrm{B}}$ that are not localized near the BChl molecules that contribute to it. That is, the structural change is spatially extended. This 
mechanism, however, is unimportant for the bacterial reaction center of Rhodopseudomonas viridis [18] and the LHC II and CP29 antenna complexes of photosystem II [19,20].

Franken, et al. [12] suggested that the just-mentioned high energy satellite holes might be mainly due to white light hole burning, [21] the white light being that of the Fourier transform spectrometer used by Johnson and Small [6] to record the pre- and post-burn spectra. The results in Figure 3 show that this is not the case. The lowest $\Delta$-absorbance spectrum is the difference between two consecutively recorded absorption spectra, each of which are the average of 100 scans taken at $1.0 \mathrm{~cm}^{-1}$ resolution. The acquisition time was $4 \mathrm{~min}$. and the intensity of the white light at the sample was $\approx 2 \mathrm{~mW} / \mathrm{cm}^{2}$. (The conditions used to record the other hole spectra reported here are the average of 200 scans.) The fractional depth of the hole at $\approx 815 \mathrm{~nm}$ is only 0.02 . Thus, the white light hole burning effect is weak. The sample was then irradiated at $\lambda_{B}=820 \mathrm{~nm}$ with a laser intensity of $10 \mathrm{~mW} / \mathrm{cm}^{2}$ for $30 \mathrm{~s}$ and the absorption spectrum recorded. The difference between it and the preceding spectrum is the middle spectrum in Figure 3. The $\mathrm{ZPH}$ at $\lambda_{\mathrm{B}}$ is just discernible and the fractional absorbance change of the weak hole at $\approx 815 \mathrm{~nm}$ is reduced to 0.01 . The top hole spectrum was obtained following additional laser irradiation at $820 \mathrm{~nm}$ with an intensity of $100 \mathrm{~mW} / \mathrm{cm}^{2}$ for $375 \mathrm{~s}$. The high energy satellite holes near 815,805 and $800 \mathrm{~nm}$ are now much more pronounced. Thus, it may be concluded that the high energy satellite holes of the top spectrum are mainly due to the structural changes that accompany hole burning at $\lambda_{B}$, in agreement with the conclusion reached by Johnson and Small [6]. This is also the case for the hole spectra shown in Figure 2 (see caption for burn intensities and burn times). 
Low energy satellite hole structure. Figure 4 shows hole spectra obtained with nine $\lambda_{B}$-values between 818.0 and $829.3 \mathrm{~nm}$. Except for the $\lambda_{B}=824.7$ and $829.3 \mathrm{~nm}$ spectra, the laser intensity and burn time used were $100 \mathrm{~mW} / \mathrm{cm}^{2}$ and $375 \mathrm{~s}$, see figure caption. For ease of discussion, the absorption spectrum is also shown (dashed curve). The deepest $\mathrm{ZPH}$ in each spectrum at $\lambda_{\mathrm{B}}$ is the resonant hole. The fractional hole depths and widths of the resonant holes are given in the figure caption. The widths of the resonant holes are determined by saturation broadening and the read resolution of $1 \mathrm{~cm}^{-1}$.

Considered first is the bottom spectrum, $\lambda_{B}=829.3 \mathrm{~nm}$. At this wavelength, excitation should be at the red edge of the absorption due to the lowest energy state that contributes to the $825 \mathrm{~nm}$ band. The dashed and solid arrows locate satellite holes displaced from the resonant hole by +18 and $-18 \mathrm{~cm}^{-1}$, respectively. That the satellite holes are symmetrically disposed relative to the resonant hole establishes that they are real- and pseudo-phonon holes (PSBH), respectively. The saturated fractional hole depth of the resonant hole is 0.75 . To a good approximation, this depth is given by $\exp (-\mathrm{S})$ [22], the Franck-Condon factor for the zero-phonon line. $\mathrm{S}$ is the Huang-Rhys factor. The result is $S=0.3$ (weak coupling), in agreement with the value reported in [6]. The absence of any observable real-PSBH in the $\lambda_{\mathrm{B}}=829.3 \mathrm{~nm}$ spectrum that lie higher in energy than the $18 \mathrm{~cm}^{-1}$ real-PSBH means that the Franck-Condon factors of their associated phonons are smaller than that of the phonon(s) associated with the $18 \mathrm{~cm}^{-1}$ hole. We return to this later. We note that the $18 \mathrm{~cm}^{-1}$ pseudo-PSBH is also indicated in the $\lambda_{\mathrm{B}}=824.7,824.0,823.0$ and $822.0 \mathrm{~nm}$ hole spectra by a solid arrow. As expected, it tracks the resonant hole at $\lambda_{\mathrm{B}}$ in a linear fashion ( $\pm 1 \mathrm{~cm}^{-1}$ uncertainty). 
Considered next is the low energy satellite hole structure in Figure 4 that consists of holes $\mathrm{a}-\mathrm{f}$. To the best of our knowledge, such rich structure has not been observed in the hole spectra of other photosynthetic complexes. (The $18 \mathrm{~cm}^{-1}$ pseudo-PSBH and holes a-d were also observed for $\mathrm{Cb}$. tepidum, results not shown.) The hole spectra of Figure 5 show, more clearly, the existence of hole $\mathrm{d}$. With $\Delta_{i}$ defined as the displacement between the resonant hole at $\lambda_{B}$ and satellite hole $i, \Delta_{i}=72,48,36,24,120$ and $165 \mathrm{~cm}^{-1}$ for $i=a-f$, respectively, as given in Table 1 . The satellite holes track the resonant hole $\left(\lambda_{\mathrm{B}}\right)$, to within \pm $1 \mathrm{~cm}^{-1}$. Analysis of the $\lambda_{\mathrm{B}}=824-820 \mathrm{~nm}$ spectra in Figure 5 revealed that the $18 \mathrm{~cm}^{-1}$ pseudo-PSBH and holes a-d attain their maximum intensity ( $\Delta$-absorbance value) when they are located near the maximum of the $825 \mathrm{~nm}$ band. From hole burning theory [22], this is consistent with the satellite hole structure being due to pseudo-PSBH, vide infra. (In what follows our use of the term phonon is meant to encompass low frequency intermolecular modes and very low frequency intramolecular modes of BChl $a$. [23]) The intensities of holes a $-\mathrm{d}$ relative to the $18 \mathrm{~cm}^{-1}$ pseudo-PSBH, as estimated on the basis of spectra where holes a $-\mathrm{d}$ and the $18 \mathrm{~cm}^{-1}$ pseudo-PSBH are near the maximum of the $825 \mathrm{~nm}$ absorption band, are given in Table 1. The $18 \mathrm{~cm}^{-1}$ hole and hole $\mathrm{d}\left(\Delta_{\mathrm{d}}=24 \mathrm{~cm}^{-1}\right)$ are considered to be due to intermolecular modes since their energies are too low to be assigned to intramolecular modes of $\mathrm{BChl} a[23]$.

The interpretation that has holes $a-f$ due to pseudo-PSBH will be referred to as interpretation $\mathrm{A}$. There is, however, an alternative interpretation (B). It has holes $\mathrm{a}, \mathrm{b}$ and $\mathrm{c}$ due to correlated, downward EET from the two higher states that contribute to the $825 \mathrm{~nm}$ absorption band. (Hole d would be assigned as a $24 \mathrm{~cm}^{-1}$ pseudo-PSBH.) The basic idea behind interpretation $\mathrm{B}$ is depicted in Figure 6 where $Z, Y, X$ label the three inhomogeneously 
broadened absorption bands that contribute to the $825 \mathrm{~nm}$ band. Not shown in the figure is the resonant hole at $\omega_{\mathrm{B}}$. Excitation at $\omega_{\mathrm{B}}$ selects two isochromats, one at the low energy side of the $\mathrm{Z}$ band and the other at the high side of the $\mathrm{Y}$ band. Isochromat $\mathrm{Z}$ can transfer energy to state Y (solid arrow) whereupon hole burning occurs to produce the center satellite hole displaced from $\omega_{\mathrm{B}}$ by $\Delta_{\mathrm{b}}$. It can also transfer to state $\mathrm{X}$ (long dashed arrow) whereupon hole burning occurs to produce the right-most hole displaced from $\omega_{\mathrm{B}}$ by $\Delta_{\mathrm{a}}$. We note that if the site excitation distribution functions (SDF) of the three states are correlated, as defined below, the $Z \rightarrow Y \rightarrow X$ EET pathway also leads to a hole at $\Delta_{\mathrm{a}}$. Isochromat $Y$ selected by $\omega_{B}$ can transfer energy to state $\mathrm{X}$, whereupon hole burning ensues to produce the left-most hole displaced from $\omega_{\mathrm{B}}$ by $\Delta_{\mathrm{c}}$. If the SDF of the three states are correlated, the satellite holes should track $\omega_{\mathrm{B}}$, i.e. $\Delta_{\mathrm{a}}, \Delta_{\mathrm{b}}, \Delta_{\mathrm{c}}$ should be independent of the $\omega_{\mathrm{B}}$-value, vide infra.

We now define what is meant by correlated. Consider states $Z$ and $X$. Let $\omega_{\mathrm{Z}}=\bar{\omega}_{\mathrm{Z}}+\mathrm{z}$ and $\omega_{\mathrm{X}}=\bar{\omega}_{\mathrm{X}}+\mathrm{x}$ be frequencies associated with their SDF. $\left(\bar{\omega}_{\mathrm{Z}}\right.$ and $\bar{\omega}_{\mathrm{X}}$ are the peak frequencies of the SDF which can be reasonably taken to be Gaussians.) For linear correlation, $\mathrm{x}=\alpha \mathrm{y}$ where $\alpha$ is a constant. Thus, excitation at $z=\omega_{\mathrm{B}}$ should result in a low energy satellite hole displaced from $\omega_{\mathrm{B}}$ by $\Delta_{\mathrm{a}}=\left(\bar{\omega}_{z}-\bar{\omega}_{\mathrm{X}}\right)+(1-\alpha) \mathrm{z}$. Perfect, positive correlation is defined by $\alpha=1$. For the sake of brevity, the SDF states of $Z$ and X for $\alpha=1$ are said to be correlated. (If $\alpha=-1$, the SDF are anti-correlated.) Thus, when the SDF of states $\mathrm{Z}$ and $\mathrm{X}$ are correlated, $\Delta_{\mathrm{a}}$ (see Figure 6) is independent of $\omega_{\mathrm{B}}$ within the $\mathrm{Z}$-band and equal to $\bar{\omega}_{Z}-\bar{\omega}_{X}$. That is, hole a should track $\omega_{\mathrm{B}}$. We emphasize that since the satellite holes track $\omega_{\mathrm{B}}$ to within $\pm 1 \mathrm{~cm}^{-1}$, the value of $\alpha$ would have to be very close to 1 . Correlation is transitive, i.e. if, for example, the SDF of states $\mathrm{Z}$ and $\mathrm{X}$ and the SDF of states 
$\mathrm{Y}$ and $\mathrm{X}$ are correlated, so are the SDF of states $\mathrm{Z}$ and $\mathrm{Y}$. Interpretation $\mathrm{B}$ of satellite holes $a, b, c$ rests on the SDF of the three states that contribute to the $825 \mathrm{~nm}$ being correlated. Correlation also means that the SDF of the three states are identical. We note that if the three states are equispaced, only two holes should be observed since $\Delta_{\mathrm{b}}=\Delta_{\mathrm{c}}$, see Figure 6 .

Arguments against interpretation $B$. As mentioned, low energy satellite hole structure as rich as that seen in Figure 4 has not been reported for other antenna complexes. Typically, the low frequency structure is confined to a single pseudo-PSBH at $\approx 20-30 \mathrm{~cm}^{-1}$ with a width of $\approx 20-30 \mathrm{~cm}^{-1}[24,25]$. (Very recently, a detailed study of the satellite structure associated with the lowest exciton level (B870) of the LH2 BChl $a$ complex of $R b$. sphaeroides revealed only a pseudo-PSBH with an energy of $16 \mathrm{~cm}^{-1}$ and a width of $10 \mathrm{~cm}^{-1}$, unpublished results.) Thus it was that we were led to consider interpretation B which has holes $\mathrm{a}, \mathrm{b}$ and $\mathrm{c}$ due to correlated EET. Holes e and $\mathrm{f}$ would be due to correlated EET from a state located at the low energy side of the intense $815 \mathrm{~nm}$ band [6] to the states that contribute to the $825 \mathrm{~nm}$ band.

There are several problems with interpretation B. First, it is not at all clear why the SDF of the three states $(X, Y, Z)$ that contribute to the $825 \mathrm{~nm}$ band should be correlated since they are most likely quite highly localized on the three subunits of the trimer. Furthermore, correlation has not been observed for other photosynthetic complexes, vide infra. The second problem arises because, with correlation, one expects that the widths of the satellite holes should equal that of the resonant hole under the conditions that: (i) the homogeneous width of the ZPL of the lower energy state that gives rise to a satellite hole is narrower than that of the ZPL at $\omega_{\mathrm{B}}$; and (ii) spectral diffusion broadening of the satellite holes due to the heat released in downward EET is negligible. The results of Ref. [11] and those in Figure 9 
establish that condition (i) is met, see Introduction. Broadening due to spectral diffusion of the type defined by (ii) has recently been observed [26,27]. However, it is over two orders of magnitude smaller than the widths of the satellite holes in the spectra shown in Figure 4 and 5. The widths of the satellite holes are given in Table 1. We conclude, therefore, that the contribution from spectral diffusion to the widths of the satellite holes is negligible. The widths of the resonant holes of the spectra shown in Figure 4 are given in the caption to that figure. Consider hole a which is relatively well resolved, Figures 4 and 5 . In the $\lambda_{B}=$ $824.7-821.0 \mathrm{~nm}$ spectra its width is significantly broader than that of the resonant hole. This is inconsistent with correlation. Furthermore, the width of hole $a$ in the spectra of Figure 4 is constant to within $\pm 2 \mathrm{~cm}^{-1}$. The average of the widths is $10 \pm 2 \mathrm{~cm}^{-1}$. For hole a to be a satellite hole due to correlated EET, its width in the top two spectra would have to be significantly broader than in the $\lambda_{B}=824.7-822.0 \mathrm{~nm}$ spectra since the width of the resonant hole in the former spectra of $7 \mathrm{~cm}^{-1}$ is a factor of 2.5 broader than those in the latter spectra. Clearly, the width of hole a does not reflect the width of the resonant hole. This is inconsistent with correlated EET. The third problem is best illustrated using hole a. With correlation and constant burn fluence conditions, its intensity ( $\Delta$ absorbance $(\Delta A)$ value) should be highest when $\omega_{\mathrm{B}}=\bar{\omega}_{\mathrm{z}}$, Figure 6 . The spectra of Figure 4 lead to a $\bar{\omega}_{\mathrm{Z}}$-value close to $12,180 \mathrm{~cm}^{-1}(821 \mathrm{~nm})$. The $\Delta \mathrm{A}$-values for $\lambda_{\mathrm{B}}=820,821$ and $822 \mathrm{~nm}$ are $0.024,0.034$ and 0.024 , respectively. Correlation also requires that the frequency at which hole a attains its maximum intensity be equal to $\bar{\omega}_{\mathrm{x}}$ which, based on the spectra in Figure 4, corresponds to a wavelength close to $825 \mathrm{~nm}$. The problem is that with the $\mathrm{X}$ and $\mathrm{Z}$ bands located near 825 and $821 \mathrm{~nm}$ and the requirement that the widths of the SDF of the $\mathrm{X}, \mathrm{Y}$ and $\mathrm{Z}$ states be equal, vide supra, an acceptable fit of the $825 \mathrm{~nm}$ absorption band was not achieved, especially on 
its low energy side (results not shown). Our attempts at fitting were quite intensive and took into account the weak electron-phonon coupling that introduces a slight asymmetry to the $\mathrm{X}$, $\mathrm{Y}$ and $\mathrm{Z}$ bands (the SDF were taken to be Gaussians). The position of the $\mathrm{Y}$ band associated with hole b was allowed to vary, within reasonable bounds, since the apparent position of hole $\mathrm{b}\left(\Delta_{\mathrm{b}}=48 \mathrm{~cm}^{-1}\right)$ may not be its true position because, for example, of interference from the anti-hole a. The intensities of the three bands were independently varied but under the constraint that the intensity of the Z-band must be significantly weaker than those of the other two bands given its location at $821 \mathrm{~nm}$ and the experimental $825 \mathrm{~nm}$ absorption band, Figure 4.

Putting aside the fitting problem, it is interesting to note that based on the results of excitonic calculations given in $[8,9]$, one would associate the Z-band with the weakly absorbing A state of the trimer with the main part of the experimental $825 \mathrm{~nm}$ band due to the more strongly allowed $\mathrm{E}$ state that is split into two components ( $\mathrm{X}$ and $\mathrm{Y}$ ) because of heterogeneity. The unequal absorption intensities of the three states then means that one is not in the strong energy disorder limit and that polarization effects associated with the $825 \mathrm{~nm}$ band should be observable. The polarized triplet-singlet difference spectra reported by van Mourik et al. [7] clearly show that significant polarization effects exist. Their results led us to perform polarized hole burning experiments as described in section 2 , the reason being that if holes a, b and $\mathrm{c}$ are due to correlated downward EET, one should observe differences in the values of their $I_{\|} / I_{\perp}$ ratio, where $I_{\|}$and $I_{\perp}$ are the intensities of the hole measured with polarization parallel and perpendicular to the burn laser polarization. The results for $\lambda_{\mathrm{B}}=822$ and $825 \mathrm{~nm}$ are shown in Figure 7 where the solid and dotted spectra correspond to $I_{i l}$ and $I_{\perp}$, respectively. The resonant holes at $\lambda_{B}$ are essentially unpolarized 
since it was necessary to burn them to saturation depth in order to be able to detect the satellite holes. The two spectra in Figure 7 show that the $18 \mathrm{~cm}^{-1}$ pseudo-PSBH and satellite holes $\mathrm{a}, \mathrm{b}$ and $\mathrm{c}$ are, for all intent and purposes, uniformly and preferentially polarized parallel to the laser polarization. Thus, the results provide no support for holes $\mathrm{a}, \mathrm{b}$ and $\mathrm{c}$ being due to correlated EET in the weak energy disorder limit. (In the strong disorder limit the polarizations of the $\mathrm{X}, \mathrm{Y}$ and $\mathrm{Z}$ states would be identical.) They are, however, consistent with holes $\mathrm{a}, \mathrm{b}$ and $\mathrm{c}$ also being pseudo-PSBH since the $\mathrm{S}_{0} \rightarrow \mathrm{Q}_{\mathrm{y}}$ electronic transition is strongly allowed, which means that the Condon approximation should be applicable. In the Condon approximation, the phonon sidebands should carry the same polarization as that of the pure electronic transition.

In summary, several reasons for satellite holes $a, b$ and $c$ not being due to correlated EET have been given. To the best of our knowledge, correlation has not been observed for a photosynthetic complex. In the absence of correlation, a low energy satellite hole that results from EET should mirror the absorption band of the state (acceptor) in which hole burning occurs. The first reported example of an absence of correlation was for the phycobilisomes of a blue-green algae [28]. For this complex, the donor and acceptor states involved in EET are the $S_{1}$-states of phycocyanin and allophycocyanin, respectively. Somewhat later an absence of correlation was reported [29] for the isolated reaction center of photosystem II of green plants where the donor state(s) are associated with Chl $a$ molecules and the acceptor state that of the pheophytin $a$ molecule active in primary charge separation. Another early example is the LH2 antenna complex of purple bacteria [30]. In that work it was shown that the SDF of the cyclic ring of $\mathrm{B} 800 \mathrm{BChl} a$ donor states are largely uncorrelated with those of the $\mathrm{B} 850$ ring of $\mathrm{BChl} a$ molecules. The reader interested in more recent examples is 
referred to Refs. $[19,20,31]$. We note that in the $\lambda_{B}=818$ and $819 \mathrm{~nm}$ spectra of Figure 4, holes a, e and $\mathrm{f}$ are superimposed on a broad hole. We assign the latter hole to uncorrelated EET from the state(s) excited at $\lambda_{\mathrm{B}}$ to the states that contribute to the $825 \mathrm{~nm}$ band.

Interpretation $A$ of the satellite structure. This interpretation has the satellite holes a,b,c,e and $\mathrm{f}$ assigned to pseudo-PSBH with peak energies of $72,48,36,120$ and $165 \mathrm{~cm}^{-1}$, respectively. (Thus, with the assignment of the 18 and $24 \mathrm{~cm}^{-1}$ satellite hole to pseudoPSBH, one has a total of seven pseudo-PSBH.) For this assignment to be convincing it is necessary to show (i) that the dependencies of the hole intensities on $\lambda_{B}$ seen in Figure 4 are consistent with the pseudo-PSBH assignment, and (ii) that reasonable assignments for the phonons associated with the PSBH exist. Considered first is the question raised by (ii).

The phonons associated with the 18 and $24 \mathrm{~cm}^{-1}$ holes are assigned as intermolecular since modes in this energy range are routinely observed in the line-narrowed spectra of $\pi$-conjugated chromophores in polymers, glasses and proteins (for a review see Ref. [25]). Such phonons have been assigned to the so-called boson peak unique to amorphous solids $[32,33]$. They are too low in energy to be assigned to intramolecular modes of chlorophyll molecules [23]. However, resonance Raman (RR) spectra of the $\mathrm{Q}_{\mathrm{y}}$-states of BChl a molecules in the reaction center of $R b$. sphaeroides and in $\mathrm{BChl} a$ films have revealed quite rich structure between $36 \mathrm{~cm}^{-1}$ and $\approx 160 \mathrm{~cm}^{-1}$. The reader is referred to Czarnecki et al. [23] for the most recent $\mathrm{RR}$ data and a review of previous RR data on the $\mathrm{Q}_{\mathrm{y}}$-states of $\mathrm{BChl} a$. Semi-empirical quantum chemical calculations of the fundamental vibrations of the BChl $a$ molecule are also presented. There is a reasonable correlation between the peak energies of holes a,b,c,e and $f$ and the RR energies which were assigned in [23] to intramolecular modes, Table 2. We say reasonable because the frequencies and relative intensities of the low 
energy intramolecular modes determined by RR depend, quite significantly, on the $\mathrm{Q}_{y}$-state being resonantly excited. Furthermore, the RR mode energies are those of the ground state while those determined by hole burning are those of the excited state. The RR bands at energies $\leqslant 130 \mathrm{~cm}^{-1}$ were assigned to vibrations that primarily involve the acetyl and methyl substituents on ring $\mathrm{I}$.

Presented next are the results of simulations that determine whether or not the dependencies of the satellite hole intensities on $\lambda_{B}$ seen in Figures 4 and 5 are consistent with their assignment to pseudo-PSBH. Because the satellite hole structure is interfered with by anti-holes and a broad underlying hole due to downward uncorrelated EET, we did not attempt to obtain exact fits; thus the focus on intensities. The results are shown in Figure 8 . Before discussing them, it is appropriate first to briefly discuss the expression of Hayes et al. [22] used in the calculations.

In the low temperature limit, the expression of Hayes et al. for the absorption spectrum following a burn for time $\tau$ reduces to

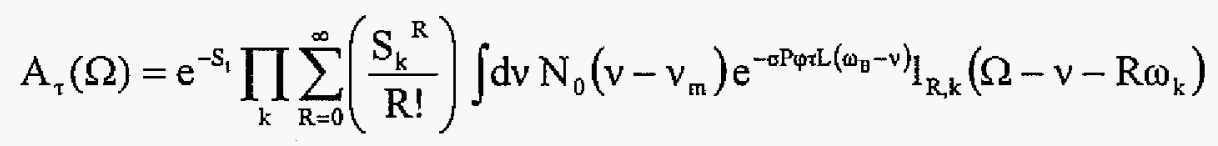

This equation does not account for anti-hole absorption. The hole burned spectrum is given by $\mathrm{A}_{\tau}(\Omega)-\mathrm{A}_{\tau=0}(\Omega) . \mathrm{S}_{\mathrm{k}}$ and $\omega_{\mathrm{k}}$ are the Huang-Rhys factor and frequency of the kth contributing mode; $S_{t}$ is the total Huang-Rhys factor $\sum_{k} S_{k}$. The zero-phonon line (ZPL) transition corresponds to $\mathrm{R}=0$. A Lorentzian with a width of $1 \mathrm{~cm}^{-1}$ (determined by the instrument resolution) was used for the ZPL. The 1-phonon, 2-phonon, ... transitions are defined by $R=1,2, \ldots$ The results in Figure 8 were obtained using Gaussian profiles for the 1-phonon transitions of the contributing modes. (Use of Lorentzian or asymmetric profiles 
yielded results that do not differ significantly.) The widths of the $R \geq 2$ profiles are obtained by folding the 1-phonon profile R-times. $N_{0}\left(v-v_{m}\right)$ is the distribution function (SDF) for the ZPL frequencies $(v) ; v_{m}$ is the peak frequency of the SDF. Since the $825 \mathrm{~nm}$ band is contributed to by three states, $\mathrm{N}_{0}$ was taken to be the sum of three Gaussians, vide infra. In the exponential of Eq. $1, \sigma$ is the integrated absorption cross-section, $\mathrm{P}$ is the photon flux and $\phi$ is the hole burning quantum yield. Since values for $\sigma$ and $\phi$ are unavailable, $\sigma \mathrm{P} \phi$ was an adjustable parameter in the calculations. The single value for $\sigma \mathrm{P} \phi$ used was obtained by fitting. Treating $\sigma \mathrm{P} \phi$ as a parameter does not affect the $\lambda_{\mathrm{B}}$-dependencies of the $\Delta \mathrm{A}$-values of the satellite holes. Finally, $\mathrm{L}\left(\omega_{\mathrm{B}}-v\right)$ is given by

$$
L\left(\omega_{B}-v\right)=e^{-S_{1}} \prod_{k} \sum_{R=0}^{\infty}\left(\frac{S_{k}{ }^{R}}{R !}\right) 1_{R, k}\left(\omega_{B}-v-R \omega_{k}\right)
$$

Here, $\omega_{\mathrm{B}}$ is the burn frequency. With $\omega_{\mathrm{B}}$ replaced by $\Omega, L(\Omega-v)$ is the absorption spectrum of a single site with a ZPL frequency equal to $v$.

The solid curve in Figure 8 is the experimental $825 \mathrm{~nm}$ absorption band. The dashed curve is the $825 \mathrm{~nm}$ band calculated using Eq. 1 with $\tau=0$. The SDF of the three contributing states were taken to carry equal intensities and widths $\left(50 \mathrm{~cm}^{-1}\right)$ and to be equispaced $\left(20 \mathrm{~cm}^{-1}\right)$. (Allowance for unequal intensities and spacings, subject to the constraint of a reasonable fit to the $825 \mathrm{~nm}$ band, resulted in hole spectra that mimic the intensity behavior of the satellite holes seen in the spectra of Figure 8). Because the Sfactors of the contributing modes are small, Table 2, the asymmetry they introduce to the calculated $825 \mathrm{~nm}$ band is smail. Except for the low energy tail, the agreement between the calculated and experimental profiles of the $825 \mathrm{~nm}$ band is quite reasonable. The sharpest and deepest hole in the calculated spectra is the resonant hole coincident with the burn 
wavelength. The Huang-Rhys factors of the contributing modes and the widths of their onephonon profiles used are given in Table 2. The hole spectra were calculated at intervals of $1 \mathrm{~nm}$ across the absorption band. Therefore, the resonant hole positions are akin to those of Figures 4 and 5. The fractional absorbance changes of the resonant holes are, from top to bottom, $0.31,0.46,0.56,0.62,0.68,0.74$ and 0.81 . These values mimic the trend set by the experimental values given in the caption to Figure 4 . The explanation for this trend is that as the burn frequency is tuned to the blue, the probability of exciting phonon transitions increases while the probability of exciting ZPL transitions decreases. This leads to a decrease and increase in the intensity of the resonant hole and pseudo-PSBH, respectively $[34,35]$. This intensity behavior is evident in the experimental spectra shown in Figures 4 and 5 and calculated spectra in Figure 8. Moreover, one observes that the intensities of the satellite holes reach their maximum values when they are located near the maximum of the $825 \mathrm{~nm}$ band. The agreement between the observed and calculated spectra is better than qualitative. To illustrate this we consider the $18 \mathrm{~cm}^{-1}$ pseudo-PSBH indicated by the solid arrows in Figures 4, 5 and 8 . When combined, the results of Figures 4 and 5 lead to relative intensity $(\triangle \mathrm{A})$ values for $\lambda_{\mathrm{B}}=828,827,826,825,824$ and $823 \mathrm{~nm}$ of $0.2,0.5,0.7,0.9,1.0$ and 0.7 , respectively. The corresponding values from Figure 8 are $0.13,0.36,0.68,0.92,1.0$, and 0.85 . We consider the agreement satisfactory given that there is considerable uncertainty in the experimental $\triangle \mathrm{A}$ values. It should be noted that real-PSBH that lie to higher energy of the resonant hole also appear in the calculated spectra. In the experimental spectra they are interfered with by the anti-hole. 
The Huang-Rhys factors and widths of the one-phonon profiles given in Table 2 can be used in the calculation of EET rates involving the states that contribute to the $825 \mathrm{~nm}$ band.

Energy transfer and spectral dynamics of the three lowest energy states. Figure 9 shows the temperature dependencies of the widths (fwhm) of ZPH burned at $822.8,824.8$ and $826.8 \mathrm{~nm}$. They are very similar to those shown in Figure 3 of Ref. [11] for Cb. tepidum where burn wavelengths of $823.0,825.0$ and $827.0 \mathrm{~nm}$ were used. (The $0.4 \mathrm{~nm}$ shift between the burn wavelengths is a consequence of the absorption maximum of the $825 \mathrm{~nm}$ band of $P$. aestuarii being at a wavelength $0.4 \mathrm{~nm}$ longer than that of $\mathrm{Cb}$. tepidum.) The discussion that follows is similar to that given in [11].

That the temperature dependence of the 822.8 and $824.8 \mathrm{~nm}$ holewidths are considerably weaker than that of the $826.8 \mathrm{~nm}$ holewidth, especially below $\approx 4 \mathrm{~K}$, suggests that the widths of the former two holes at $1.8 \mathrm{~K}$ are dominated by a temperature-independent contribution, one that is attributed to downward energy transfer. Preliminary analysis of the $826.8 \mathrm{~nm}$ data points showed that they approximately follow a $\mathrm{T}^{\alpha}$ dependence with $\alpha \sim 1.3$. This power law is unique to pure dephasing/spectral diffusion due to two-level systems (TLS) of glasses and proteins. Such a temperature dependence has recently been reported for the lowest energy $Q_{y}$-state of the photosystem II reaction center [36], the LHC II antenna complex of photosystem II [19], and the lowest exciton level of the B850 molecules in the LH2 antenna complex of purple bacteria [37]. However, the hole widths for $\lambda_{B}=826.8 \mathrm{~nm}$ are not solely determined by the TLS. This follows because of the apparent convergence of the holewidths for the three burn wavelengths at higher temperatures. That is, if the $826.8 \mathrm{~nm}$ holewidths were due only to TLS, they should add to the energy transfer 
contributions to the widths of the 822.8 and $824.8 \mathrm{~nm}$ holes. The EET kinetics can be safely assumed to be temperature independent for $\mathrm{T} \leq 10 \mathrm{~K}$. This line of argument becomes more convincing when the holewidths in Figure 9 are fit by

$$
\Gamma_{\text {hole }}\left(\mathrm{cm}^{-1}\right)=\Gamma_{0}+2 A T^{\alpha}
$$

where $\Gamma_{0}$ is temperature independent and the second term is due to TLS-induced spectral dynamics. The $826.8 \mathrm{~nm}$ data were fit first (solid curve), resulting in $\Gamma_{0}=0.015 \mathrm{~cm}^{-1}, \alpha=$ 1.3 and $A=0.0056$ (Table 3 ). The lifetime of the lowest energy state is $2 \mathrm{~ns}[10,11]$. The lifetime contribution to $\Gamma_{0}$ is given by $2\left(2 \pi T_{1} c\right)^{-1}$, where $T_{1}$ is the lifetime and $c$ is the speed of light in the units of $\mathrm{cm} \cdot \mathrm{s}^{-1}$. The lifetime contribution is $0.005 \mathrm{~cm}^{-1}$, leaving $\approx 0.010 \mathrm{~cm}^{-1}$ of $\Gamma_{0}$ to be explained. As in Ref. [11], we attribute the $0.010 \mathrm{~cm}^{-1}$ to spectral diffusion and/or a small contribution to the holewidth from the next higher energy state near $824.8 \mathrm{~nm}$. For fitting of the 824.8 and $822.8 \mathrm{~nm}$ data, $\alpha$ was fixed at 1.3 , leaving $\Gamma_{0}$ and $A$ as the only adjustable parameters. That $A=0.0056$ for the three $\lambda_{B}$-values, Table 3 , supports our analysis procedure since the spectral dynamics from TLS should be the same for the three subunits of the trimer. As in refs 9,10 the $\Gamma_{0}$-values obtained for $\lambda_{\mathrm{B}}=824.8$ and $822.8 \mathrm{~nm}$ are assigned to lifetime broadening due to downward EET. They lead to the $T_{1}$-values of 99 and 26 ps given in Table 3.

For comparison, the values of $\mathrm{T}_{\mathrm{l}}, \mathrm{A}$ and $\alpha$ for $\mathrm{Cb}$. tepidum are also given in Table 3 . That they are similar to those for $P$. aestuarii is not surprising given that the structures of the two species are very similar as are their $825 \mathrm{~nm}$ absorption bands, Figure 1. The differences in the above values may not be significant since the $\lambda_{\mathrm{B}}$-values used for $P$. aestuarii may not be exactly equivalent to those used for $C b$. tepidum (keeping in mind that the $825 \mathrm{~nm}$ band is due to three overlapping and inhomogeneously broadened bands). 
With reference to Figure 6 , the $\mathrm{T}_{1}$-values of $P$. aestuarii for $\lambda_{\mathrm{B}}=822.8$ and $824.8 \mathrm{~nm}$ (823.0 and $825.0 \mathrm{~nm}$ for $C b$. tepidum) are assigned to downward EET from the $\mathrm{Z}$ and $\mathrm{Y}$ states, respectively. For the latter state there is only the $\mathrm{Y} \rightarrow \mathrm{X}$ pathway while for the former there are the $Z \rightarrow Y$ and $Z \rightarrow X$ pathways. At this point it seems most likely that these EET pathways should be describable in terms of weak coupling nonadiabatic transfer theory. The electron-phonon coupling parameters that define the spectral densities in the rate expression are given in Table 2. It will be interesting to see whether or not they can explain why the transfer rate for the $\mathrm{Z}$ state is four times higher than that of the $\mathrm{Y}$ state. Such calculations, which take into account the effects of structural heterogeneity, are now in progress.

\section{Conclusions}

The nonphotochemical hole burning spectra and spectral dynamics data reported for the FMO complex of $P$. aestuarii pertain mainly to the lowest energy absorption band at $825 \mathrm{~nm}$ which is contributed to by three states. These states stem from the lowest energy state of the subunit of the trimer. The splittings between them, which can be no greater than about $30 \mathrm{~cm}^{-1}$, appear to be dominated by structural heterogeneity. Earlier hole burning studies had shown that the widths of the three underlying absorption bands are mainly inhomogeneously broadened $\left(\approx 50 \mathrm{~cm}^{-1}\right)$ at liquid helium temperatures. The two main objectives of our experiments were to interpret the rich low energy satellite hole structure observed when the burn wavelength $\left(\lambda_{B}\right)$ is located in the $825 \mathrm{~nm}$ band and to determine the excitation energy transfer (EET) kinetics for the two higher energy states that contribute to the $825 \mathrm{~nm}$ band. 
The $\lambda_{\mathrm{B}}$-dependent hole spectra obtained are the most detailed yet reported for an antenna complex. They reyeal low energy satellite holes at $18,24,36,48$ and $72 \mathrm{~cm}^{-1}$, as measured relative to the resonant zero-phonon hole at $\lambda_{\mathrm{B}}$. The last four holes are designated as holes $\mathrm{d}, \mathrm{c}, \mathrm{b}$ and $\mathrm{a}$, respectively, in Table 1 which also includes the energies of two higher energy satellite holes. The 18 and $24 \mathrm{~cm}^{-1}$ holes are assigned to pseudo-phonon sideband hole (PSBH) due to intermolecular phonons. Two interpretations for holes $\mathrm{c}, \mathrm{b}$ and a at 36 , 48 and $72 \mathrm{~cm}^{-1}$ were considered. The interpretation that has them due to correlated EET from the two higher energy states that contribute to $825 \mathrm{~nm}$ band could be rejected. Thus, the FMO complex is yet another example where the site distribution functions of different states exhibit little correlation. Based on theoretical simulations of the $\lambda_{\mathrm{B}}$-dependence of the hole spectra, we conclude that the above holes are pseudo-PSBH and that the $36,48,72,120$ and $165 \mathrm{~cm}^{-1}$ phonons are most likely due to $\mathrm{BChl} a$ intramolecular modes. Their HuangRhys factors and one-phonon profile widths are given in Table 2.

The temperature dependencies $(1.8-10 \mathrm{~K})$ of the widths of zero-phonon holes burned in the $825 \mathrm{~nm}$ band of $P$. aestuarii at $\lambda_{\mathrm{B}}=822.8,824.8$ and $826.8 \mathrm{~nm}$ were found to be similar to those of $C b$. tepidum, Table 3 . These wavelengths should be close to the absorption maxima of the three states that contribute to the $825 \mathrm{~nm}$ band, $Z, Y$ and $X$ in Figure 2. Pure dephasing/spectral diffusion due to the glass-like two-level systems of the protein is operative and identical for the three states. When this contribution to the holewidths is taken into account the time constants for excitation energy transfer from the $\mathrm{Y}$

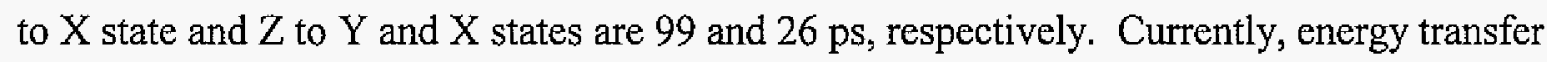
calculations are in progress that utilize the electron-phonon coupling parameters given in Table 2 with due account being given to the effects of energy disorder. 


\section{Acknowledgments}

Research at the Ames Laboratory was supported by the Division of Chemical Sciences, Office of Basic Energy Sciences, U.S. Department of Energy. Ames Laboratory is operated for USDOE by Iowa State University under Contract W-7405-Eng-82. We are indebted to Professor T. Aartsma of Leiden University for providing the samples of the FMO complex from $P$. aestuarii.

\section{References}

1. Fenna, R. E.; Matthews, B. W.; Olson, J. M.; Shaw, E. K. J. Mol. Biol. 1974, 84, 231.

2. Blankenship, R. E.; Olson, J. M.; Miller, M. In Anoxygenic Photosynthetic Bacteria; Blankenship, R. E., Madigan, M. T., Bauer, C. E., Eds.; Kluwer: Dordrecht, 1995; pp. 399-435.

3. Savikhin, S.; Buck, D. R.; Struve, W. S. J. Phys. Chem. B 1998, 102, 5556.

4. Lu, X.; Pearlstein, R. M. Photochem. Photobiol. 1993, 57, 86.

5. Li, Y. F.; Zhou, W.; Blankenship, R. E.; Allen, J. P. J. Mol. Biol. 1997, 271, 456.

6. Johnson, S. G.; Small, G. J. J. Phys. Chem. 1991, 95, 471.

7. van Mourik, F.; Verwijst, R. R.; Mulder, J. M.; van Grodelle, R. J. Phys. Chem. 1994, 98, 10307.

8. Gülen, D. J. Phys. Chem. 1996, 100, 17684.

9. Louwe, R. J. W.; Vrieze, T.; Aartsma, T. J.; Hoff, A. J. J. Phys. Chem. B 1997, 101, 11280.

10. Louwe, R. J. W.; Aartsma, T. J. J. Phys. Chem. 1997, 101, 7221.

11. Rätsep, M.; Blankenship, R. E.; Small, G. J. J. Phys. Chem. B 2000, 103, 5736.

12. Franken, E. M.; Neerken, S.; Louwe, R. J. W.; Amesz, J.; Aartsma, T. J. Biochemistry 1998, 37, 5046. 
13. Holt, S. C.; Conti, S. F.; Fuller, R. C. J. Bacteriol. 1996, 91, 311.

14. Francke, C.; Amesz, J. Photosyn. Res. 1997, 53, 137.

15. Shu, L.; Small, G. J. J. Opt. Soc. Am. 1992, 9, 733 and refs. therein.

16. Shu, L.; Small, G. J. J. Opt. Soc. Am. 1992, 9, 724.

17. Kim, W.-H.; Reinot, T.; Hayes, J. M.; Small, G. J. J. Phys. Chem. 1995, 99, 7300.

18. Reddy, N. R. S.; Kolaczkowski, S. V.; Small, G. J. J. Phys. Chem. 1993, 97, 6934.

19. Pieper, J.; Rätsep, M.; Jankowiak, R.; Irrgang, K.-D.; Voigt, J.; Renger, G.; Small, G. J. J. Phys. Chem. A 1999, 103, 2412.

20. Pieper, J.; Irrgang, K.-D.; Rätsep, M.; Voigt, J.; Renger, G.; Small, G. J. Photochem. Photobiol. 2000, in press.

21. Milanovich, N.; Hayes, J. M.; Small, G. J. Mol. Cryst. Liq. Cryst. 1996, 291, 147.

22. Hayes, J. M.; Lyle, P. A.; Small, G. J. J. Phys. Chem. 1994, 98, 7337.

23. Czarnecki, K.; Diers, J. R.; Chynwat, V.; Erickson, J. P.; Frank, H. A.; Bocian, D. F. J. Am. Chem. Soc. 1997, 119, 415 and refs. therein.

24. Jankowiak, R.; Small, G. J. In Photosynthetic Reaction Centers, Deisenhofer, J. and Norris, J., Eds.; Academic Press: New York, 1993, p. 133.

25. Small, G. J. Chem. Phys. 1995, 197, 239.

26. den Hartog, F. T. H.; Bakker, M. P.; Silbey, R. J.; Völker, S. Chem. Phys. Lett. 1998, 297,314 .

27. den Hartog, F. T. H.; van Papendrecht, C.; Silbey, R. J.; Völker, S. J. Chem. Phys. $1999,110,1010$.

28. Köhler, W.; Friedrich, J.; Fischer, R.; Scheer, H. J. Chem. Phys. 1988, 89, 871.

29. Tang, D.; Jankowiak, R.; Seibert, M.; Yocum, C. F.; Small, G. J. J. Phys. Chem. $1990,94,6519$.

30. Reddy, N. R. S.; Small, G. J.; Seibert, M.; Picorel, R. Chem. Phys. Lett. 1991, 181, 391. 
31. Rätsep, M.; Johnson, T. W.; Chitnis, P. R.; Small, G. J. J. Phys. Chem. B, 1999, 104, 836.

32. Ahn, J. S.; Kanematsu, Y.; Kushida, T. Phys. Rev. B 1993, 48, 9058.

33. Kanematsu, Y.; Ahn, J. S.; Kushida, T. Phys. Rev. B 1993, 48, 9066.

34. Lee, I. J.; Hayes, J. M.; Small, G. J. J. Chem. Phys. 1989, 91, 3413.

35. Lyle, P. A.; Kolaczkowski, S. V.; Small, G. J. J. Phys. Chem. 1993, 97, 6926 and refs. therein.

36. den Hartog, E. T. H.; Vacha, F.; Lock, A. J.; Barber, J.; Dekker, J. P.; Völker, S. J. Phys. Chem. B 1998, 102, 9174.

37. Wu, H.-M.; Rätsep, M.; Lee, I.-J.; Cogdell, R. J.; Small, G. J. J. Phys. Chem. B 1997, 101,7654 . 
Table 1. Spectral Data of the Low Energy Satellite Holes, $P$. aestuarii.

\begin{tabular}{|c|ccc|}
\hline Hole & $\Delta\left(\mathbf{c m}^{-\mathbf{1}}\right)^{\mathbf{b}}$ & $\mathbf{\Delta A ^ { \mathbf { c } }}$ & Width $\left.\mathbf{( c m}^{-1}\right)^{\mathbf{d}}$ \\
\hline Pseudo-PSBH & \\
a & 18 & 0.08 & 10 \\
$\mathrm{~b}$ & 72 & 0.023 & 10 \\
$\mathrm{c}$ & 48 & 0.008 & 5 \\
$\mathrm{~d}$ & 36 & 0.02 & 9 \\
$\mathrm{e}$ & 24 & 0.004 & 5 \\
$\mathrm{f}$ & 120 & 0.014 & 9 \\
& 165 & 0.015 & 10 \\
\hline
\end{tabular}

${ }^{\text {a}} \mathrm{PSBH} \equiv$ phonon sideband hole.

${ }^{\mathrm{b}}$ Displacement measured relative to resonant zero-phonon hole at burn wavelength.

${ }^{\mathrm{c}} \Delta$-absorbance value for hole when located near the maximum of the $825 \mathrm{~nm}$ band. ${ }^{\mathrm{d}}$ Full width at half maximum, $\pm 2 \mathrm{~cm}^{-1}$. 
Table 2. Parameter Values Used in Calculations and BChl $a$ Vibrational Energies.

\begin{tabular}{|c|c|c|c|}
\hline $\begin{array}{c}\text { Phonon } \\
\text { Energy }\left(\mathrm{cm}^{-1}\right)^{\mathrm{b}}\end{array}$ & $\mathbf{S}^{\mathbf{c}}$ & Width $\left(\mathrm{cm}^{-1}\right)^{d}$ & $\begin{array}{l}\text { RR Modes }^{\mathrm{c}} \\
\left(\mathrm{cm}^{-1}\right)\end{array}$ \\
\hline 18 & $\overline{0.24}$ & 10 & n.o. ${ }^{f}$ \\
\hline $72(a)$ & 0.12 & 7.5 & 56,72 \\
\hline $48(b)$ & 0.04 & 5.0 & 56 \\
\hline $36(\mathrm{c})$ & 0.12 & 10 & 36 \\
\hline 24(d) & 0.05 & 5.0 & n.o. \\
\hline $120(\mathrm{e})$ & 0.09 & 10 & 118 \\
\hline $165(\mathrm{f})$ & 0.09 & 10 & 163 \\
\hline
\end{tabular}

${ }^{a}$ The 120 and $165 \mathrm{~cm}^{-1}$ modes were not included in the calculations. Their S-values were determined using the value of 0.12 for the $72 \mathrm{~cm}^{-1}$ mode and the $\lambda_{B}=820,819$, $818 \mathrm{~nm}$ hole spectra shown in Figure 4 and the spectrum for $\lambda_{B}=817 \mathrm{~nm}$ (not shown).

${ }^{\mathrm{b}}$ The letters in parentheses identify the phonon frequencies with the low energy satellite holes seen in the spectra of Figure 4.

${ }^{\mathrm{c}} \mathrm{S} \equiv$ Huang-Rhys factor.

${ }^{\mathrm{d}}$ Full-width at half-maximum of the one-phonon profile.

${ }^{\mathrm{e}} \mathrm{BChl} a$ vibrational energies from resonance Raman experiments on the $\mathrm{Q}_{\mathrm{y}}$-states of the $R b$. sphaeroides reaction center, from Ref. [23].

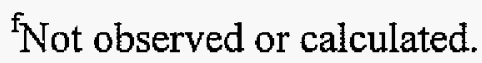


Table 3. Analysis of Zero-Phonon Holewidths of the Three States that Contribute to the $825 \mathrm{~nm}$ Band.

\begin{tabular}{|cccc|}
\hline $\boldsymbol{\lambda}_{\mathbf{B}}(\mathbf{n m})$ & $\mathbf{T}_{\mathbf{1}}(\mathbf{p s})^{\mathbf{a}}$ & $\mathbf{A}\left(\mathbf{c m}^{-\mathbf{1}} \mathbf{K}^{-\mathbf{a}}\right)^{\mathbf{b}}$ & $\boldsymbol{a}^{\mathbf{b}}$ \\
\hline \multicolumn{4}{c|}{ P. aestuarii } \\
822.8 & 26 & .0056 & 1.3 \\
824.8 & 99 & .0056 & 1.3 \\
826.8 & $2.10^{3 \mathbf{c}}$ & .0056 & 1.3 \\
& Cb. tepidum (from ref 11) & \\
823.0 & 32 & 0.0070 & 1.3 \\
825.0 & 135 & 0.0070 & 1.3 \\
827.0 & $2.10^{3}$ & 0.0070 & 1.3 \\
\hline
\end{tabular}

${ }^{a}$ Lifetimes of three contributing states.

${ }^{b}$ Parameters associated with pure dephasing/spectral diffusion due to two-level systems, see Eq. 3.

From Refs. $[10,11]$. 


\section{Figure Captions}

Figure 1. The $4.2 \mathrm{~K} \mathrm{Q}$-absorption spectrum of the FMO complex from P. aestuarii (solid curve) and $C b$. tepidum (dashed curve).

Figure 2. Hole burned spectra of the FMO complex from $P$. aestuarii obtained with burn wavelength $\left(\lambda_{B}\right)=823.0 \mathrm{~nm}$ at $4.2 \mathrm{~K}$. For convenience the absorption spectrum is also shown. From top to bottom the burn intensity and burn time used were: $50 \mathrm{~mW} / \mathrm{cm}^{2}, 30 \mathrm{~s} ; 100 \mathrm{~mW} / \mathrm{cm}^{2}, 75 \mathrm{~s}$; and $100 \mathrm{~mW} / \mathrm{cm}^{2}, 375 \mathrm{~s}$. The deepest and sharpest hole in each spectrum is the resonant zero-phonon hole at $\lambda_{B}=823.0 \mathrm{~nm}$. The solid arrows locate the $18 \mathrm{~cm}^{-1}$ pseudo-phonon sideband hole (PSBH). The satellite holes $a, b$ and $c$ correspond to those indicated in Figures 4 and 5 . The broad and higher energy holes are the result of the structural changes that accompany hole burning in the $825 \mathrm{~nm}$ absorption band. The structural changes alter the energies of the states that contribute to the higher energy absorption band, see text.

Figure 3. Spectra of the FMO complex from $P$. aestuarii related to the white light hole burning effect, $\mathrm{cf}$. text for discussion. For convenience the absorption spectrum is also shown. The excessive noise in the spectra near $815 \mathrm{~nm}$ is due to the high absorbance. $\mathrm{T}=4.2 \mathrm{~K}$.

Figure 4. The dependence of the $4.2 \mathrm{~K}$ hole burned spectrum of the FMO complex from $P$. aestuarii on burn wavelength $(818.0-829.3 \mathrm{~nm})$. The deepest hole in each spectrum is the resonant zero-phonon hole located at $\lambda_{\mathrm{B}}$. The burn intensities and times used to obtain the $\lambda_{B}=818-824 \mathrm{~nm}$ spectra were $100 \mathrm{~mW} / \mathrm{cm}^{2}$ and $375 \mathrm{~s}$. The intensity and time for the $\lambda_{\mathrm{B}}=824.7$ and $829.3 \mathrm{~nm}$ were $125 \mathrm{~mW} / 180 \mathrm{~s}$ and $250 \mathrm{~mW} / 660 \mathrm{~s}$, respectively. The solid arrows locate the $18 \mathrm{~cm}^{-1}$ pseudo-PSBH; the dashed arrow in the $\lambda_{B}=829.3 \mathrm{~nm}$ locates the real-PSBH due to the $18 \mathrm{~cm}^{-1}$ phonon. The energies of holes $a-f$ as measured relative to $\lambda_{B}$ are given in Table 1. The dashed curve is the absorption spectrum. The fractional hole depths of the 
resonant hole are, from top to bottom, $0.34,0.34,0.42,0.49,0.43,0.68,0.75$, $0.61,0.75$, respectively. The corresponding holewidths are $6.8,6.7,4.2,3.0,3.0$, $2.6,1.6,2.0 \mathrm{~cm}^{-1}$.

Figure 5. Low energy satellite hole structures obtained with $\lambda_{B}=824.0-828.0 \mathrm{~nm}$, $\mathrm{T}=4.2 \mathrm{~K}$. Burn intensity and time were $\sim 200 \mathrm{~mW} / \mathrm{cm}^{2}$ and up to $1500 \mathrm{~s}$. The deepest hole in each spectrum is the resonant zero-phonon coincident with the burn wavelength $\left(\lambda_{\mathrm{B}}\right)$. The spectra more clearly establish the existence of hole $\mathrm{d}$. The dashed curve is the $825 \mathrm{~nm}$ absorption band.

Figure 6. Schematic of correlated downward energy transfer that results in satellite holes a, $\mathrm{b}$ and $\mathrm{c}$ displaced from the burn frequency $\omega_{\mathrm{B}}$ by $\Delta_{\mathrm{a}}, \Delta_{\mathrm{b}}$, and $\Delta_{\mathrm{c}}$. $\mathrm{Z}, \mathrm{Y}$ and X denote the three $\mathrm{Q}_{\mathrm{y}}$-states that contribute to the $825 \mathrm{~nm}$ band.

Figure 7. Polarized hole burned spectra of the FMO complex from $P$. aestuarii obtained with burn wavelengths 822.0 and $825.0 \mathrm{~nm}, \mathrm{~T}=4.2 \mathrm{~K}$. Dark solid spectrum: horizontal polarization and dotted spectrum: vertical polarization.

Figure 8. Calculated hole spectra for different burn wavelengths $(822-828 \mathrm{~nm})$. The deepest hole in each spectrum is the resonant hole at $\lambda_{\mathrm{B}}$. The arrow locates the $18 \mathrm{~cm}^{-1}$ pseudo-PSBH. Satellite holes a-d correspond to those shown in Figure 5. The experimental (solid curve) and calculated absorption (dashed curve) spectra are also shown. The values of Huang-Rhys factors (S) and widths of the one-phonon profiles used are given in Table 2; see text for further discussion.

Figure 9. Temperature dependence of zero-phonon holes burned at $822.8,824.8$ and $826.8 \mathrm{~nm}$. The solid curves are fits obtained using Eq. 3; see text for further discussion, 


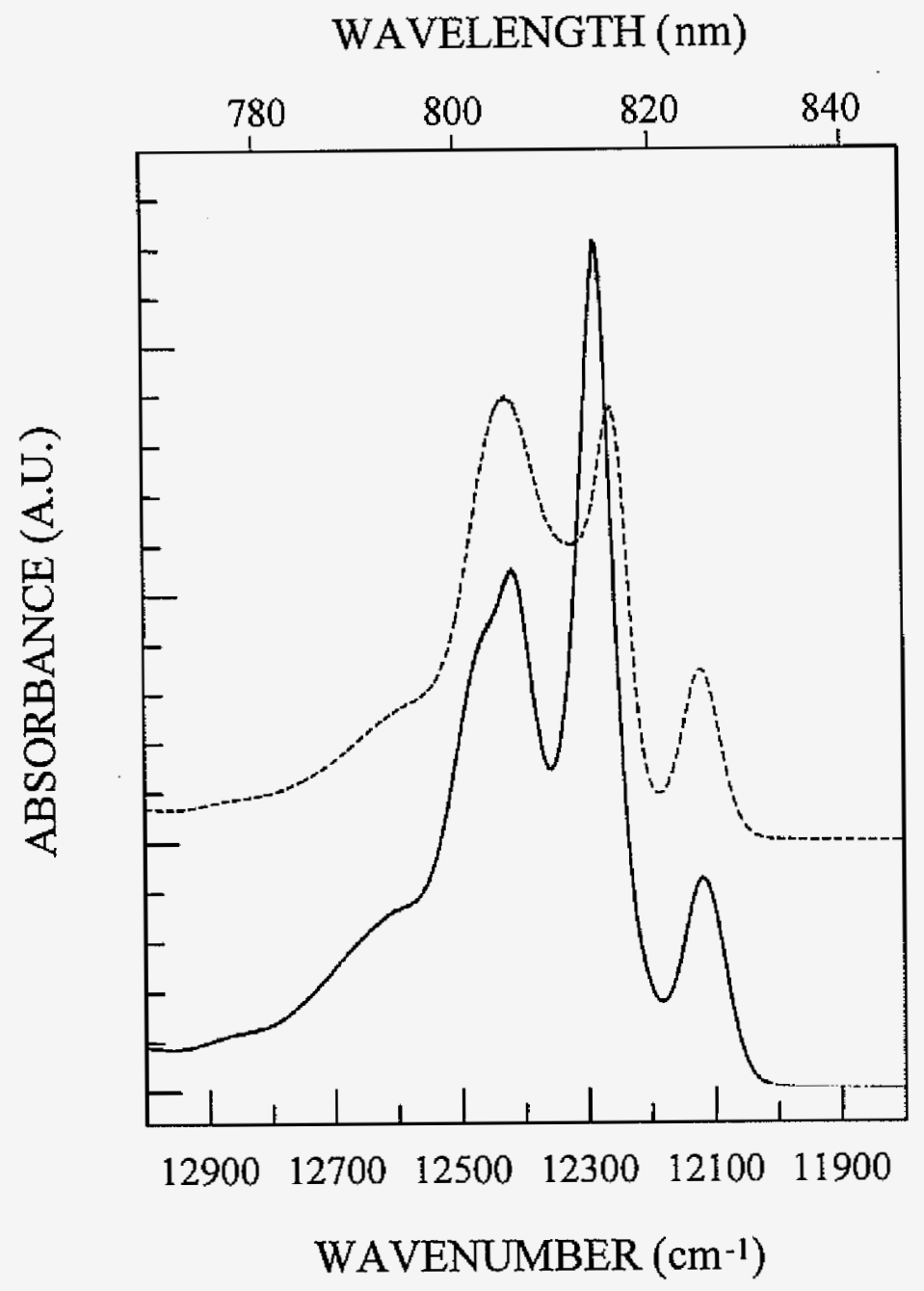

Figure 1. 


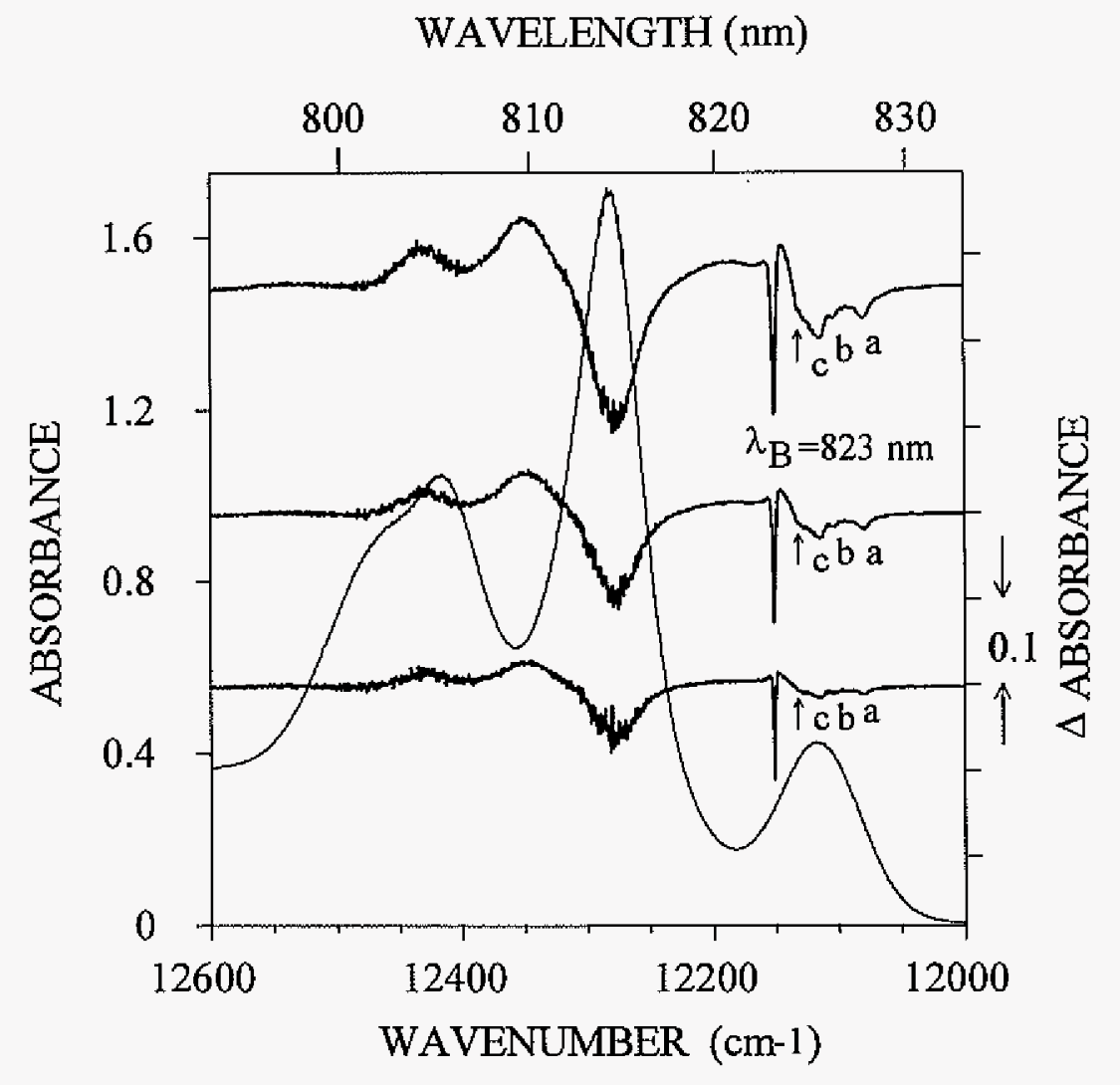

Figure 2. 


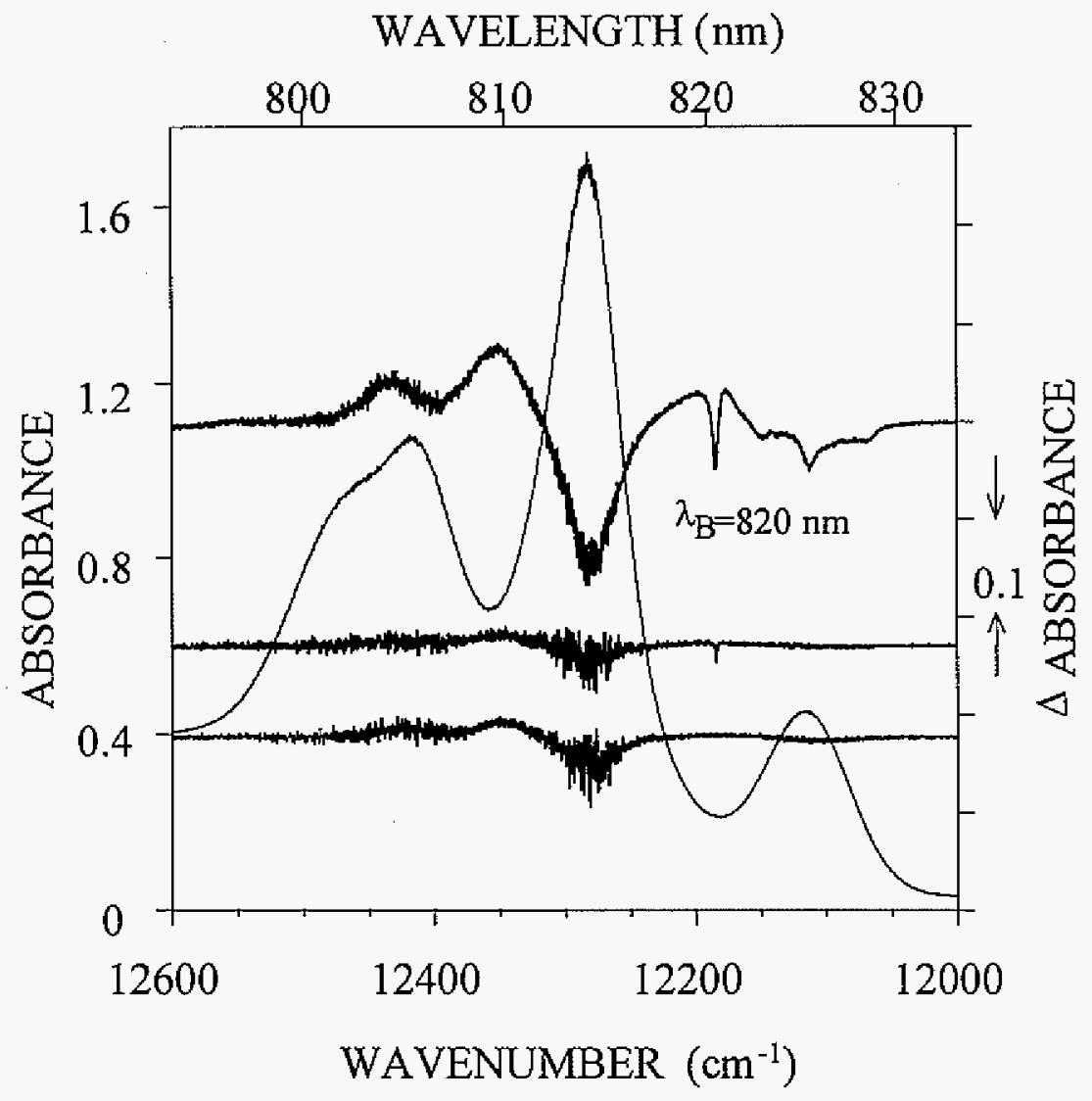

Figure 3. 


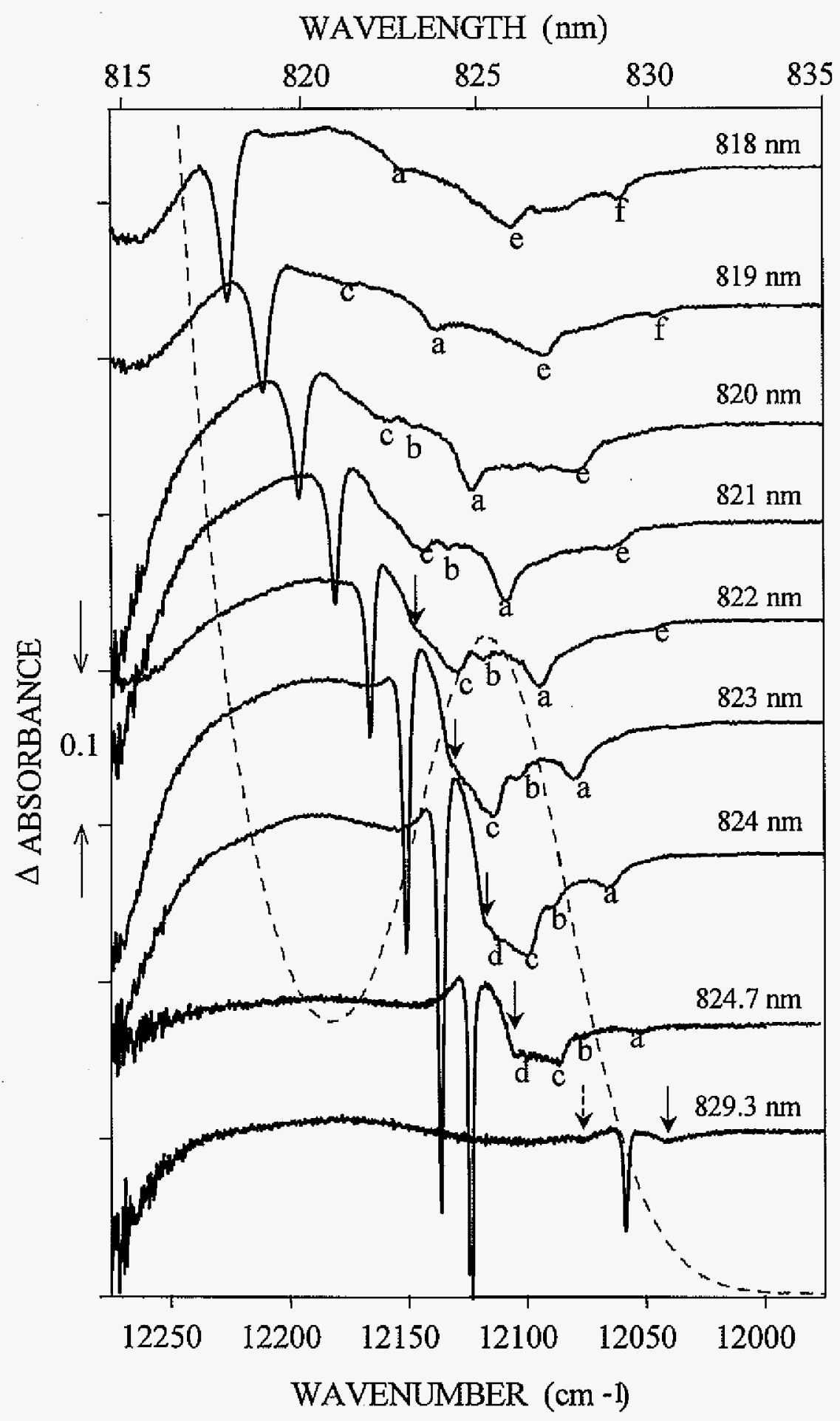

Figure 4. 


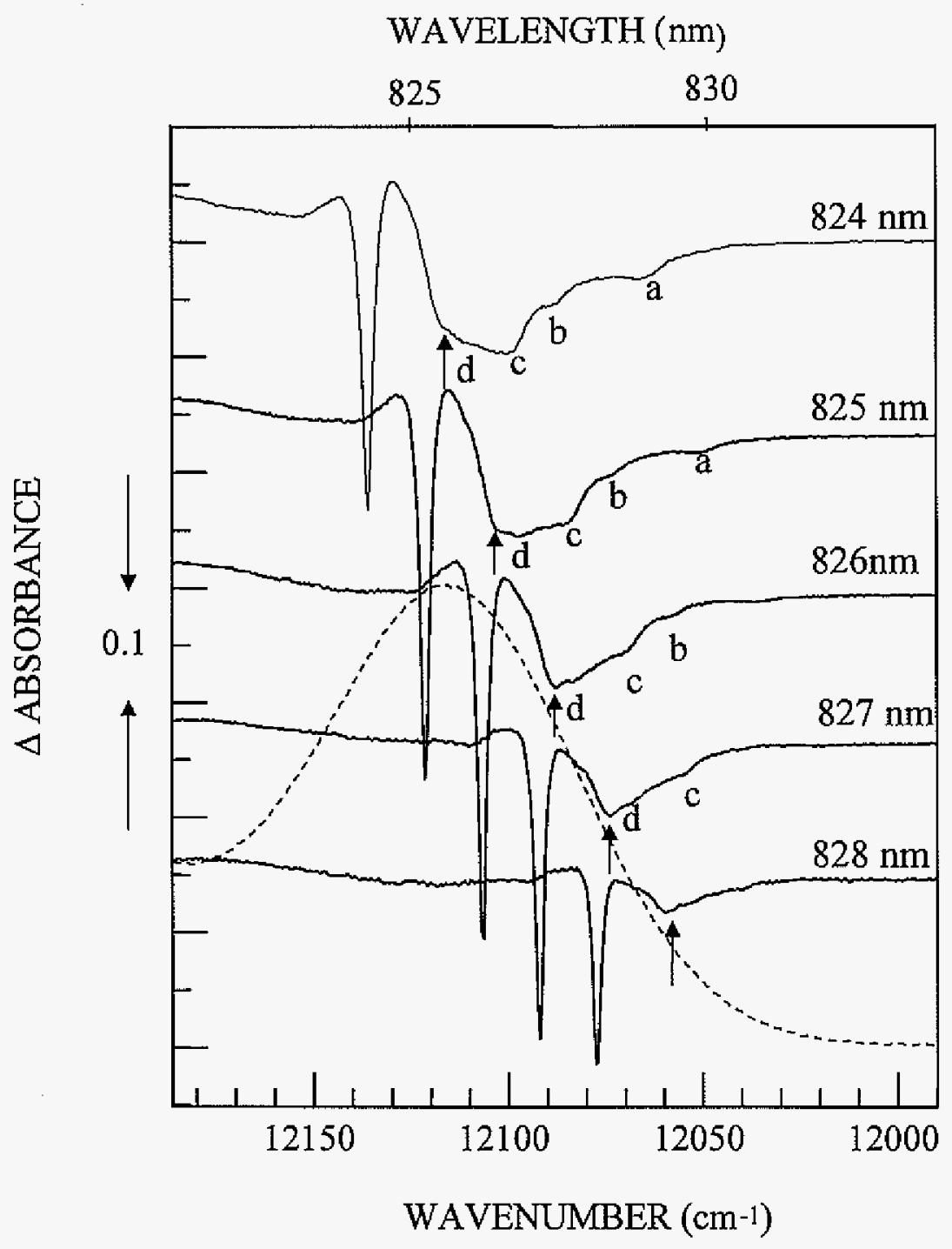

Figure 5. 


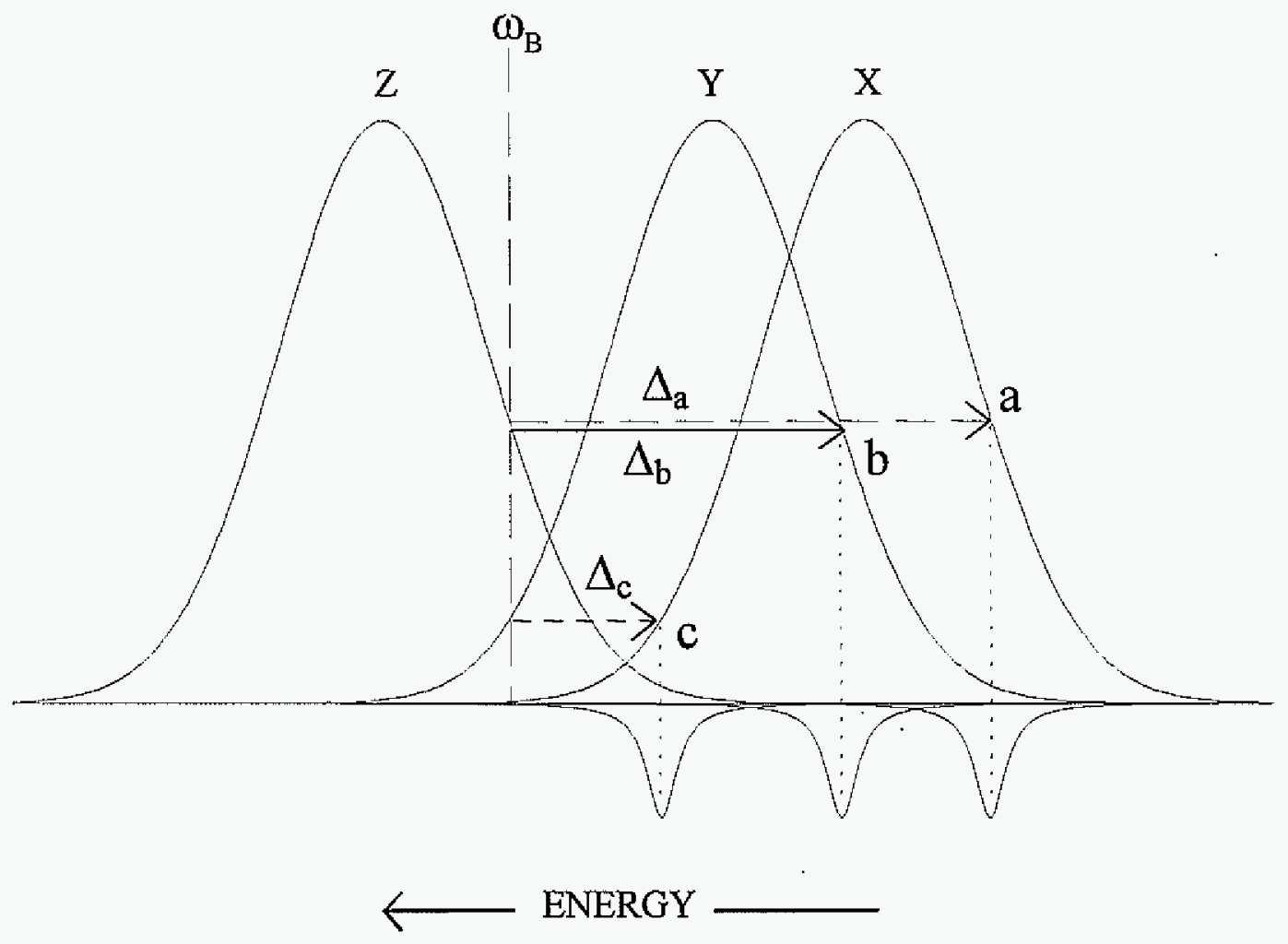

Figure 6. 


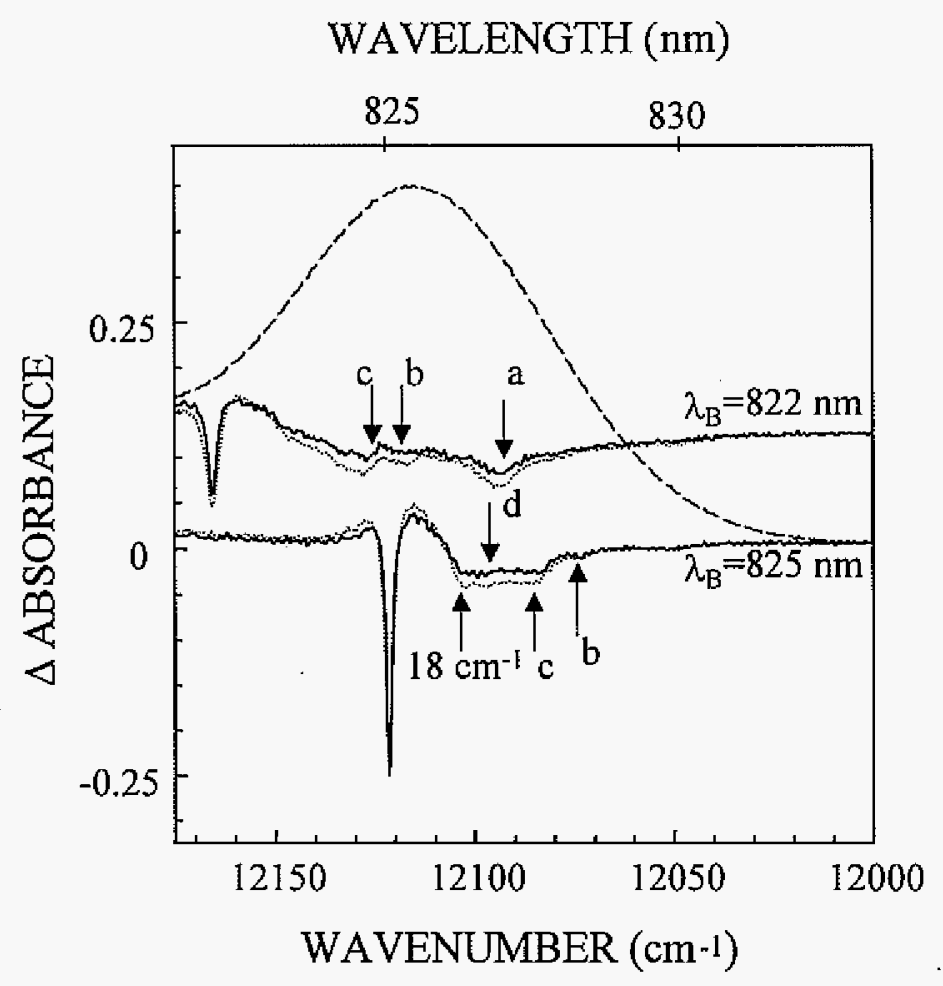

Figure 7. 


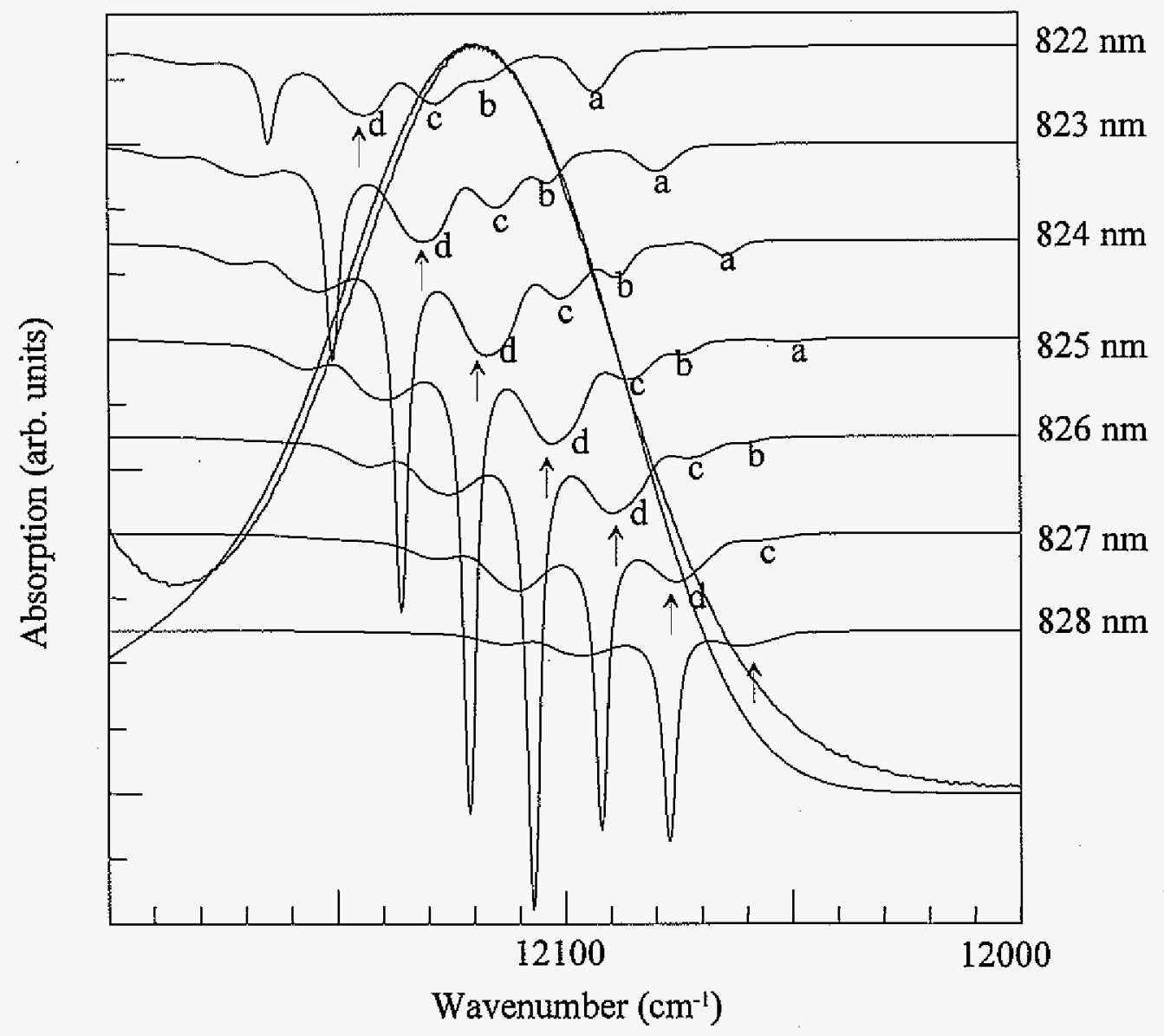

Figure 8. 


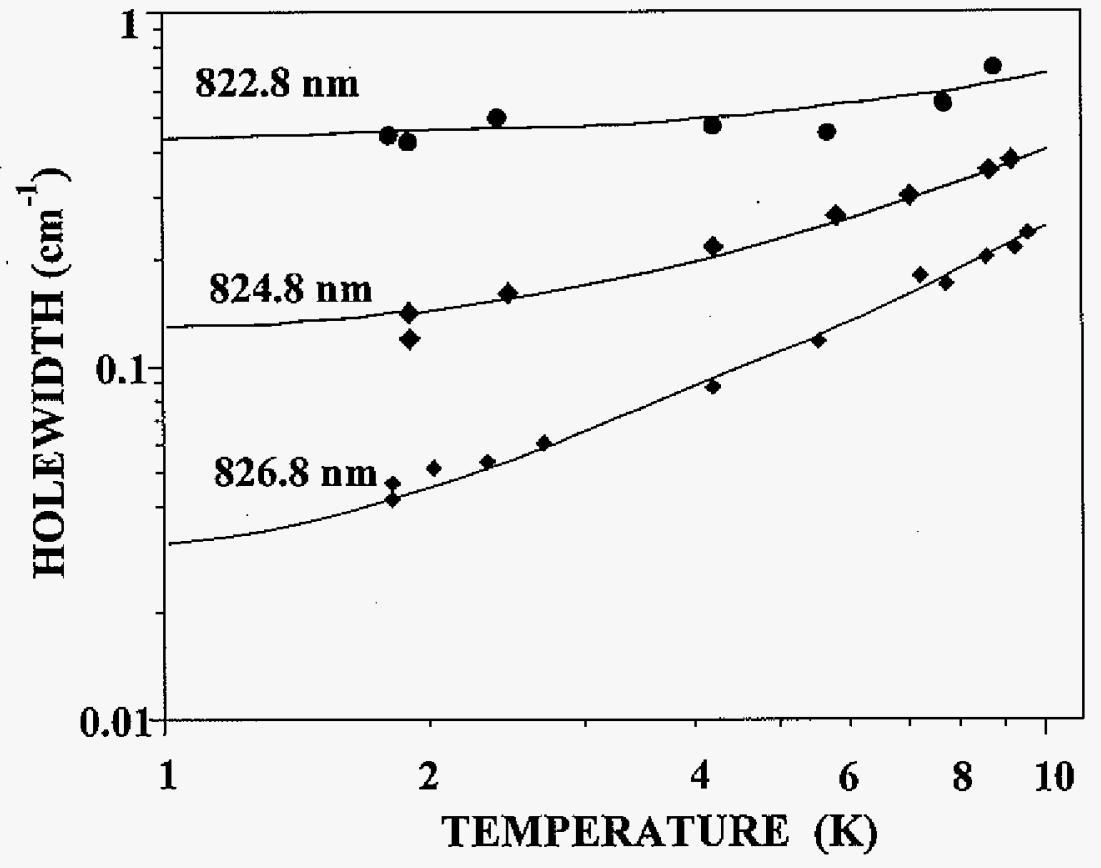

Figure 9. 


\title{
CHAPTER 4. ENERGY TRANSFER DYNAMICS IN LH2 COMPLEXES OF RHODOPSEUDOMONAS ACIDOPHILA CONTAINING ONLY ONE B800 MOLECULE
}

\author{
A paper published in the Journal of Physical Chemistry B 2001, 105, 7049. \\ S. Matsuzaki, V. Zazubovich, N. J. Fraser, R. J. Cogdell, and G. J. Small
}

\begin{abstract}
Nonphotochemical hole burning (NPHB) and femtosecond pump-probe experiments have previously shown that following their excitation, the B800 molecules of the LH2 complex of Rhodobacter sphaeroides and Rhodopseudomonas (Rps.) acidophila relax by two channels. The decay channel observed for excitation on the low energy side of the B800 absorption band is due to $\mathrm{B} 800 \rightarrow \mathrm{B} 850$ excitation energy transfer which occurs in $\sim 2 \mathrm{ps}$ at $4 \mathrm{~K}$. The additional decay channel becomes detectable for excitation on the high energy side of the B800 band. The mechanisms that have been proposed for this channel are: (i) vibrational relaxation following excitation of vibronic levels associated with the $\mathrm{Q}_{\mathrm{y}}$-states of either the B800 or B850 bacteriochlorophyll $a$ molecules; (ii) direct excitation of upper B850 exciton levels, followed by downward relaxation within the B850 manifold; (iii) intraband B800-B800 energy transfer involving only the B800 molecules; and (iv) downward energy transfer of initially excited mixed B800-B850 states. Presented here are NPHB results for intact LH2 and B800-deficient LH2 complexes of Rps. acidophila (strain 10050). The latter contain only one B800 molecule, rather than nine. In sharp contrast with the intact LH2 complex, the zero-phonon holewidths for B800-deficient LH2 complex were observed to be independent of the location of the burn frequency within the $\mathrm{B} 800$ band, $3.2 \pm 0.3 \mathrm{~cm}^{-1}$ at
\end{abstract}


$4 \mathrm{~K}$. This finding and others eliminate the first two of the above mechanisms. It is argued that the third mechanism is highly unlikely. It is proposed that mixed B800-B850 states are mainly responsible for the additional decay channel. These mixed states could undergo downward relaxation to $\mathrm{B} 800$ levels that are mainly "B800" in character and to levels that are mainly "B850" in character.

\section{Introduction}

The $\mathrm{Q}_{\mathrm{y}}\left(\mathrm{S}_{1}\right)$ electronic structure and excitation energy transfer (EET) processes of the light harvesting $(\mathrm{LH}) 1$ and $\mathrm{LH} 2$ complexes of purple bacteria have long been of much interest [1]. The determination in 1995 [2] of the X-ray structure of LH2 from Rps. acidophila (strain 10050) at a resolution of $2.5 \AA$ stimulated even more activity. LH2 is also referred to as $\mathrm{B} 800-\mathrm{B} 850$ because of the location of the bacteriochlorophyll $a(\mathrm{BChl} a)$ absorption bands at $\sim 800$ and $850 \mathrm{~nm}$ at room temperature. The B800 and B850 molecules are located at the cytoplasmic and periplasmic sides of the membrane, respectively. The structure revealed that $\mathrm{LH} 2$ carries $\mathrm{C}_{9}$ symmetry with the B800 ring a 9-mer of monomers and the $\mathrm{B} 850$ ring a 9-mer of dimers whose BChl $a$ monomers are energetically inequivalent. (The symmetry is reduced to $\mathrm{C}_{8}$ in Rhodospirillum molischianum [3].) Whereas the excitonic

coupling between neighboring $\mathrm{B} 850$ molecules is strong, $\sim+300 \mathrm{~cm}^{-1}$, the corresponding coupling between $\mathbf{B 8 0 0}$ molecules is weak, $\sim-20 \mathrm{~cm}^{-1}$ (see Refs. [4,5]). Structural data for LHI of Rs. rubrum point to it being a cyclic 16-mer of BChl $a$ dimers with an inner diameter large enough to house the reaction center complex [6]. LH1 is often referred to as B875. These structures, together with results from femtosecond and nonphotochemical hole burning (NPHB) experiments, electronic structure and EET calculations have significantly improved 
our understanding of the light-driven processes in the antenna system of purple bacteria (see the recent review by Sundström et al. [7]). In that article the importance of energy disorder, which stems from the structural heterogeneity of proteins, for understanding excitonic structure and EET is stressed. Energy disorder (diagonal and/or off-diagonal) and excitonphonon coupling determine the extent to which the aggregate $\mathrm{BChl} a$ states are delocalized. The prevailing view is that the states of the B800 ring are highly localized on individual $\mathrm{BChl} a$ molecules while those of the B850 ring are not [7]. However, the extent of delocalization of the latter continues to be controversial (see Ref. [8] and references therein). We present here hole burning and absorption results that shed new light on the intraband $\mathrm{B} 800-\mathrm{B} 800$ and interband $\mathrm{B} 800 \rightarrow \mathrm{B} 850$ EET processes. The latter has been extensively studied by ultra-fast pump-probe spectroscopy in Rhodobacter (Rb.) sphaeroides [9-13] and Rps. acidophila (strain 10050) [14-16], referred to hereafter as just Rps. acidophila. At room temperature the $\mathrm{B} 800 \rightarrow \mathrm{B} 850$ transfer times for both species are similar, $\sim 0.7 \mathrm{ps}$. This time increases to $1.6 \mathrm{ps}$ in the low temperature limit $[11,16]$. Persistent NPHB has also been used to determine the $\mathrm{B} 800 \rightarrow \mathrm{B} 850$ transfer times at liquid helium temperatures for $R b$. sphaeroides [16-20] and Rps. acidophila [16,21]. The widths of the zero-phonon holes (ZPH) burned at the maximum and to lower energy of the B800 band were found to be constant and led to transfer times of 1.9 and 2.5 ps for Rps. acidophila and $R b$. sphaeroides, respectively. Wu et al. [16] reported transfer times determined by femtosecond pump-probe and NPHB spectroscopies of $1.6 \pm 0.2 \mathrm{ps}$ and $1.9 \pm 0.2 \mathrm{ps}$, respectively, for Rps. acidophila in the low temperature limit. Taken as a whole, the time domain and NPHB results establish that the lifetimes of the B800 molecules excited at the low energy side of the $\mathrm{B} 800$ band are determined by $\mathrm{B} 800 \rightarrow \mathrm{B} 850 \mathrm{EET}$ at low temperatures. 
Of particular relevance to this paper is that the ZPH widths for both $R b$. sphaeroides [20] and Rps. acidophila [16] were observed to increase (approximately linearly) as the burn wavelength $\left(\lambda_{\mathrm{B}}\right)$ was tuned to the blue of the maximum of the B800 band. For example, the holewidth at $\lambda_{\mathrm{B}}=790 \mathrm{~nm}$ for $R b$. sphaeroides is $7.7 \mathrm{~cm}^{-1}$ which corresponds to a lifetime of $700 \mathrm{fs}$, over three times shorter than the lifetime for $\lambda_{\mathrm{B}} \geq 798 \mathrm{~nm}$ (maximum of the B800 band). The "additional" decay channel for B800 was also detected at low temperatures in pump-probe experiments (see review by Sundström et al. [7]). For example, $783 \mathrm{~nm}$ excitation at the blue edge of the B800 band of Rps. acidophila (B800 absorption maximum at $803 \mathrm{~nm}$ ) resulted in a lifetime of $400 \mathrm{fs}$ [16]. A $400 \mathrm{fs}$ component was not detected in the photobleaching of B850. The additional decay channel has also been observed for single complexes of Rps. acidophila [22].

Wu et al. [16] considered several explanations for the additional decay channel - that it is due to: (i) vibrational relaxation of $\mathrm{BChl} a$ modes excited at the blue side of the $\mathrm{B} 850$ band that build on either the B800 or B850 origin bands; (ii) downward relaxation of directly excited upper exciton levels of the B850 ring (such levels for Rps. acidophila have been predicted to lie in the near vicinity of the $\mathrm{B} 800$ band and be weakly absorbing $[4,5,23])$; and (iii) downward B800-B800 intraband EET. With regard to (iii), it was suggested that the "blue" B800 states might be mixed B800-B850 states involving certain upper B850 levels. de Caro et al. [20] favored interpretation (iii) with only B800 molecules involved. However, the EET calculations of Wu et al. [16] do not support this. Van Oijen et al. [22], aware of those calculations, favored interpretation (i). Wu et al. [16], on the basis of Franck-Condon factor arguments, considered that interpretation quite unlikely. The results of their pumpprobe experiments argued against interpretation (ii). In the end, they favored interpretation 
(iii) with the above mixed B800-B850 states playing a major role. Clearly, the question as to the nature of the additional $\mathrm{B} 800$ decay channel was left unsettled by the above studies.

We present here the results of NPHB experiments on LH2 complexes of Rps. acidophila that are almost deficient in B800 molecules but carry the full complement of B850 molecules that are weakly perturbed by the procedure used to remove the B800 molecules. The B800-deficient LH2 enabled us to determine the EET kinetics from a single B800 molecule to the B850 ring. The B800 lifetime was found to be independent of excitation wavelength within the B800 band and equal to $3.2 \pm 0.3 \mathrm{ps}$ at $4 \mathrm{~K}$. Implications of this finding for the aforementioned mechanisms of the additional B800 decay channel are considered.

\section{Experimental}

The LH2 complexes of Rps. acidophila were isolated and purified as in Ref. [24]. After dilution in glycerol:water solution (2:1 by volume) containing $0.1 \% \mathrm{LDAO}$ detergent in water $(\mathrm{pH} 8,20 \mathrm{mM}$ Tris $\mathrm{HCl})$ samples were contained in polypropylene tubes (i.d. $=$ $9 \mathrm{~mm}$ ) and cooled in a Janis 8-DT liquid helium cryostat for ambient pressure studies. A Lake Shore temperature controller (Model 330) was used for stabilization and measurement of temperature.

The procedure for removing the $\mathrm{B} 800$ molecules from the $\mathrm{LH} 2$ complex has been extensively described elsewhere [25]. Briefly, B800 molecules were released by incubating a LH2 sample containing Triton BG-10 at a pH of 4.75 at $30^{\circ} \mathrm{C}$ for $1 \mathrm{~h}$. B850 complexes lacking B800 molecules were then purified by ion exchange chromatography using 
phosphocellulose as the absorbant. This procedure leads to almost complete removal of B800 molecules [25].

The hole burning apparatus is described in detail elsewhere [26]. In short, a Bruker IFS 120 HR Fourier transform spectrometer was used to record preburn and postburn absorption spectra at a resolution of $0.5 \mathrm{~cm}^{-1}$. The burn laser was a Coherent $899-21$ Ti:sapphire laser (linewidth of $0.07 \mathrm{~cm}^{-1}$ ) pumped by $15 \mathrm{~W}$ Coherent Innova $200 \mathrm{Ar}$ ion laser. The reported widths of the $\mathrm{ZPH}$ burned in the $\mathrm{B} 800$ band were obtained from $\mathrm{ZPH}$ with a fractional hole depth less than 0.07 . Burn intensities and times are given in the figure captions.

\section{Results and Discussion}

Figure 1 shows the $4.2 \mathrm{~K}$ absorption spectra of Rps. acidophila for intact LH2 (a) and B800-deficient $\mathrm{LH} 2$ (b). The $\mathrm{B} 800$ and $\mathrm{B} 850$ bands of the former are located at 802.9 and $868.7 \mathrm{~nm}$, respectively, and carry widths (fwhm) of $135 \mathrm{~cm}^{-1}$ and $210 \mathrm{~cm}^{-1}$, respectively. These values are similar to those reported in $[27,28]$ for intact LH2 complexes isolated and purified by the same procedure. The spectrum of B800-deficient LH2 is similar to that given in [25]. The B850 band is red-shifted by $1.2 \mathrm{~nm}$ relative to the value for intact $\mathrm{LH} 2$ and its width is broader by $25 \mathrm{~cm}^{-1}$. A more detailed comparison of the two $\mathrm{B} 850$ bands is given later. The integrated intensity of the weak band at $799.7 \mathrm{~nm}$ relative to that of the $\mathrm{B} 800$ band of intact LH2 is 0.025 upon equalization of the peak intensities of the $B 850$ bands.

Assignment of the $799.7 \mathrm{~nm}$ band. Gall et al. [25] suggested that this band may be due to residual $\mathrm{B} 800$ molecules bound to the $\beta$-apoproteins of LH2 or a weakly absorbing upper exciton level of the $\mathrm{B} 850$ ring. (The possibility that it is due to free $\mathrm{BChl} a$ monomer 
could be excluded since the monomer absorbs at $\sim 770 \mathrm{~nm}\left(13,000 \mathrm{~cm}^{-1}\right)$. The spectra in the inset of Figure 1 and other results eliminate the latter possibility. Spectrum $b$ is a magnified view of the $12,500 \mathrm{~cm}^{-1}$ region of spectrum $\mathrm{b}$. Spectrum $\mathrm{c}$ is that of the same sample after storage at $0^{\circ} \mathrm{C}$ in the dark for one week. While the $\mathrm{B} 850$ band was unaffected by storage (result not shown), the intensity of the $799.7 \mathrm{~nm}$ band decreased by a factor of $\sim 4$. This is inconsistent with an upper exciton level assignment. We attribute the diminuation in intensity to glycerol which readily extracts B800 (but not B850) molecules from the LH2 complex (unpublished results). Thus, in studying "intact" LH2 complexes of Rps. acidophila it is important to add glycerol last and cool quickly to low temperatures. We note that B800deficient LH2 can be reconstituted with a variety of chlorophyll-type molecules $[25,29]$. Additional support for the assignment of the $799.7 \mathrm{~nm}$ band to residual $\mathrm{B} 800$ molecules in the LH2 complex is provided by the observation that its peak position is invariant to temperature between $4 \mathrm{~K}$ and room temperature (results not shown). This unusual temperature independence is precisely that observed for the $\mathrm{B} 800$ band of intact $\mathrm{LH} 2$ complexes of Rps. acidophila and Rb. sphaeroides [28]. In contrast, the B850 band exhibits a strong linear blue shift for $\mathrm{T} \gtrsim 150 \mathrm{~K}, 0.9 \mathrm{~cm}^{-1} / \mathrm{K}$ for Rps. acidophila [28]. The same shift rate was observed for the $\mathrm{B} 850$ band of B800-deficient $\mathrm{LH} 2$ (data not shown). This shifting provides additional support for the $799.7 \mathrm{~nm}$ band not being due to an upper exciton level for the $\mathrm{B} 850$ ring. With the assignment of the $799.7 \mathrm{~nm}$ band to residual $\mathrm{B} 800$ molecules in the LH2 complex, the diminuation factor of 0.025 (vide supra) results in there being $0.2 \mathrm{~B} 800$ molecules per LH2 complex. Under the reasonable assumption that the kinetics of B800 dissociation from the LH2 complex is the same for all complexes in the bulk sample, the 
most probable situation is that $\sim 80 \%$ of the complexes are devoid of B 800 molecules while $\sim 20 \%$ contain 1 B 800 molecule.

B800 zero-phonon holewidths for B800-deficient LH2. These widths are shown in the inset of Figure 2. The experimental uncertainty is estimated at $\pm 0.3 \mathrm{~cm}^{-1}$. Thus, the widths are independent of burn (excitation) wavelength located within the $\mathrm{B} 800$ band. This independence is in striking contrast with the results for intact LH2 for which the holewidth increases for $\lambda_{\mathrm{B}}$ shorter than that of the maximum of the $\mathrm{B} 800$ band, cf. Introduction. This dependence for intact LH2 of Rps. acidophila is shown in Figure 3. The average of the holewidths for B800-deficient LH2 is $3.2 \pm 0.3 \mathrm{~cm}^{-1}$, which corresponds to $\mathrm{B} 800 \rightarrow \mathrm{B} 850$ EET time of 3.2 ps. We attribute this lifetime, which is somewhat longer than that for intact LH2 (vide infra), to LH2 complexes that contain only 1 B800 molecule. The independence of the holewidth on $\lambda_{\mathrm{B}}$ for B800-deficient LH2 eliminates two of the interpretations for the additional decay channel of B800 presented in the Introduction. They are that it is due to vibrational relaxation of BChl $a$ modes excited at the high energy side of the B800 band; and excitation of upper exciton levels of the B850 ring that undergo downward relaxation (here we consider that these levels are determined by a Hamiltonian that involves only the B850 molecules). Elimination of the latter follows since deletion of B800 molecules results in only a small red-shift (1.2 nm) of the B850 band and does not significantly affect the BChl $a-$ $\mathrm{BChl} a$ couplings of the $\mathrm{B} 850$ ring (vide infra). That is, the locations of the B850 upper exciton levels should be close to those of intact LH2. (We note that circular dichroism studies of a B800-free mutant of $R b$. sphaeroides led to assignment of an upper exciton level located on the high energy side of the B800 band [30].) Similarly, the vibronic structure associated with the $\mathrm{B} 850$ exciton manifold of $\mathrm{B} 800$-deficient $\mathrm{LH} 2$ should be very similar to 
that of intact LH2. Since the B800-B800 nearest neighbor excitonic coupling is weak relative to the diagonal energy disorder, the $\mathrm{B} 800$ excitations are expected to be highly localized on individual B800 molecules [7]. Thus, the low frequency vibrational structure that builds on the $\mathrm{B} 800$ origin transition of a single $\mathrm{B} 800$ molecule in B800-deficient $\mathrm{LH} 2$ should also be similar to that of intact LH2. Therefore, the first of the above two interpretations also appears very unlikely.

We conclude, therefore, that the additional B800 decay channel is due to intraband B800-B800 EET. In LH2 complexes possessing only one B800 molecule this channel is eliminated, explaining, therefore, the $\lambda_{\mathrm{B}}$-independence of the B800 holewidths in Figure 2. In what follows we consider the question of the mechanism by which B800-B800 transfer occurs.

Mechanistic aspects of $\mathbf{B 8 0 0 - B 8 0 0}$ transfer. Although several groups have calculated the kinetics of B800 $\rightarrow$ B850 EET [16,31-35], there has been only one reported calculation on the kinetics of B800-B800 transfer [16]. In that work, Wu et al. attempted to explain holewidth data for the B800 band of the type shown here in Figure 3. Before discussing their results, a few remarks regarding current thinking on B800-B800 transfer are in order: (i) to a good approximation B800-B800 transfer can be viewed as occurring between states localized on individual BChl $a$ molecules; (ii) the dipole-dipole coupling approximation for calculating B800-B800 excitonic couplings should be accurate since $\mathrm{Mg} . . \mathrm{Mg}$ distances between $\mathrm{BChl} a-\mathrm{BChl} a$ molecules are $\geq 21 \AA$; (iii) while the nearest neighbor coupling appears to be sufficiently strong to explain a B800-B800 transfer time of $\sim 1$ ps for optimal spectral overlap between the donor fluorescence and acceptor absorption spectra [16], coupling between non-nearest neighbors is not [36]; and (iv) the $\mathrm{B} 800 \rightarrow \mathrm{B} 850$ 
transfer time should be weakly dependent on excitation frequency within the B800 band. Item (iv) follows from the data of Refs. [16,31] which show that large (hundreds of $\mathrm{cm}^{-1}$ ) pressure-induced changes in the B800-B850 energy gap have only a weak effect on the $\mathrm{B} 800 \rightarrow \mathrm{B} 850$ transfer time. The results presented here for B800-deficient LH2 are entirely consistent with this. Thus, the contribution from B800-B800 transfer to the holewidths can be obtained by subtracting the constant holewidth on the red side of the B800 band from the measured widths. The resulting residual holewidths for the data in Figure 3 are given by the right ordinate in that figure. The width subtracted was $4.0 \mathrm{~cm}^{-1}$; the average of the widths for the four lowest $\omega_{\mathrm{B}}$-values is $4.0 \pm 0.2 \mathrm{~cm}^{-1}$, which corresponds to a $\mathrm{B} 800 \rightarrow \mathrm{B} 850$ transfer time of $2.5 \pm 0.1 \mathrm{ps}$ (a holewidth of $1 \mathrm{~cm}^{-1}$ corresponds to a $10 \mathrm{ps}$ lifetime). The residual holewidth of $22 \mathrm{~cm}^{-1}$ corresponds to the solid circle data point (from Ref. [16]) in the figure. Importantly, it lies on the straight line defined by the four diamond data points. The $22 \mathrm{~cm}^{-1}$ width corresponds to a lifetime of $0.4 \mathrm{ps}$ which two-color pump-probe spectra indicated is due to downward B800 intraband transfer. For $\omega_{\mathrm{B}}$-values $\lesssim$ the frequency of the $\mathrm{B} 800$ band maximum, this transfer is too slow, relative to $\mathrm{B} 800 \rightarrow \mathrm{B} 850$ transfer, to be measurable. Returning to the aforementioned paper by Wu et al. [16], they employed the weak coupling, nonadiabatic rate expression of Kolaczkowski et al. [37] which requires, as input, the electron-phonon coupling parameters for the $\mathrm{B} 800 \mathrm{~S}_{0} \rightarrow \mathrm{Q}_{\mathrm{y}}$ optical transition. It is this coupling that governs the spectral overlap between the donor fluorescence and acceptor absorption spectra. The hole burned spectra revealed coupling to phonons with a peak energy of $\omega_{\mathrm{m}} \sim 30 \mathrm{~cm}^{-1}$, a Huang-Rhys factor $S$ of 0.3 (weak coupling) and a one-phonon profile width of $\sim 30 \mathrm{~cm}^{-1}$. The results of their calculations, which employed a reasonable value for the nearest neighbor coupling, revealed that a $\sim 1 \mathrm{ps} \mathrm{B800-B800} \mathrm{transfer} \mathrm{time} \mathrm{is}$ 
possible when the donor-acceptor energy gap is close to $\omega_{m}$ in value. However, the spectral overlap (density) for gaps larger than $\sim 30 \mathrm{~cm}^{-1}$ decreases quite rapidly. For gaps of 50,60 and $80 \mathrm{~cm}^{-1}$ the spectral overlaps, relative to the value for the $30 \mathrm{~cm}^{-1}$ gap, are $0.15,0.04$, and 0.001 , respectively. As a result, the $\omega_{\mathrm{B}}$-dependence of the holewidth shown in Figure 3 , which is very similar to that observed for Rb. sphaeroides [20], could not be explained. (In addition, their calculations predicted a strong temperature dependence for $\mathrm{B} 800-\mathrm{B} 800$ transfer which is not observed [38].) To expand on their reasoning we consider the function

$$
F\left(\omega_{B}\right)=\int_{\omega_{B}}^{\omega_{B}} d \omega \rho(\omega) D\left(\omega_{B}-\omega\right)
$$

which governs downward B800-B800 energy transfer for the localized excitations. $\rho(\omega)$ is the site excitation energy distribution function (SDF) which can be taken to be a Gaussian. $\mathrm{D}\left(\omega_{\mathrm{B}}-\omega\right)$ is the square of the spectral overlap factor for sites excited at $\omega_{\mathrm{B}}$ that transfer downward to sites at $\omega$. It is instructive to consider first the case where $D\left(\omega_{\mathcal{B}}-\omega\right)$ is smooth and slowly varying over the frequency range defined by the SDF. This situation would arise when the electron-phonon coupling is strong and/or involves phonons whose frequencies cover a broad range. One can then remove $\mathrm{D}\left(\omega_{\mathrm{B}}-\omega\right)$ from the integral with the result being that $F\left(\omega_{B}\right)$ has a sigmoidal shape. It is shown in the inset of Figure 3 for $\rho(\omega)$ a Gaussian with $\mathrm{fwhm}=120 \mathrm{~cm}^{-1}$, a value appropriate for the $\mathrm{B} 800$ band. $\mathrm{F}\left(\omega_{\mathrm{B}}\right)$ represents the fraction of B800 molecules that lie lower in energy than $\omega_{\mathrm{B}}$. Although the straight experimental line may correspond to the linear region of the sigmoidal curve, it is shifted substantially to the left of the latter. Thus, the prediction that the $\mathrm{B} 800-\mathrm{B} 800$ transfer time for $\omega_{\mathrm{B}}$ located at the maximum of the SDF (solid curve in inset) should be twice that for $\omega_{\mathrm{B}}$ located at its blue edge is at odds with the experimental data. Furthermore, the experimental holewidths show 
no indication of leveling off at high values of $\omega_{\mathrm{B}}$. This is also the case for $R b$. sphaeroides [20]. The situation worsens when $D\left(\omega_{B}-\omega\right)$ in Eq. 1 is not smooth and slowly varying, but rather a "window" function centered at $\omega=\omega_{\mathrm{B}}-\Delta$. (Based on the values of the electronphonon coupling parameters used in [16], $\mathrm{D}\left(\omega_{\mathrm{B}}-\omega\right)$ would be peaked at $\sim 60 \mathrm{~cm}^{-1}$ and carry a width of $\sim 50 \mathrm{~cm}^{-1}$ ). In this case one expects, for example, that leveling off of the holewidth at higher frequencies should onset at a lower frequency. In this regard it is instructive to consider that $\mathrm{D}\left(\omega_{\mathrm{B}}-\omega\right)$ is much sharper than $\rho(\omega)$ and peaked at $\omega_{\mathrm{B}}-\Delta$. Then $F\left(\omega_{B}\right) \sim \rho\left(\omega_{B}-\Delta\right)$, from which it is apparent that the differences between the widths of holes burned on the low and high energy sides of the B800 band should not be nearly as large as those indicated by the data.

The above analysis leads us to conclude that the holewidth data cannot be understood in terms of downward B800-B800 energy transfer that involves only the B800 molecules. We propose, as in Ref. [16], that the data are the result of mixed B800-B850 states with the degree of mixing being greater on the high energy side of the B800 band than on the low energy side. The relevant B850 levels would be the upper exciton levels of the B850 ring and be biased towards the high energy side of the B800 band. Excitonic structure calculations were performed in the nearest dimer-dimer coupling approximation with a Hamiltonian defined by [23]

$$
\begin{gathered}
E_{1}^{j}=e_{1}+2 V_{1} \cos (2 \pi j / n) ; j=0,1, \ldots, 8 \\
E_{u}^{j}=e_{u}+2 V_{u} \cos (2 \pi j / n)
\end{gathered}
$$

and

$$
\mathrm{H}_{\mathrm{ul}}^{\mathrm{j}}=2 \mathrm{~V}_{\mathrm{ul}} \cos (2 \pi \mathrm{j} / \mathrm{n})
$$


where $e_{1}$ and $e_{u}$ are the energies of the lower and upper levels of the basic dimer of the B850 ring. (The basic dimer is that of the protomer of the B850 ring as defined in Ref. [4].) Eqs. 2 and 3 define the energies associated with the exciton manifolds spawned by the I and $u$ dimer levels; $V_{I}$ and $V_{u}$ are the nearest dimer-dimer coupling constants. In the absence of energy disorder, intermanifold mixing is restricted to levels of the same j-value and given by Eq. 4 . As explained in Ref. [39], the $V_{l}, V_{u}$ and $V_{u l}$ dimer-dimer couplings can be determined from the monomer-monomer coupling energies. The values of Sauer et al. [4] for the latter yield $V_{u}=100 \mathrm{~cm}^{-1}, V_{1}=-200 \mathrm{~cm}^{-1}$, and $V_{u l}=130 \mathrm{~cm}^{-1}$ (slightly rounded off values) [23]. The coupling energies between the monomers of the basic dimer is $\sim 300 \mathrm{~cm}^{-1}$. Thus, we use $e_{u}-e_{1}=600 \mathrm{~cm}^{-1}$ as in Ref. [23]. The energies of the B850 exciton levels obtained are shown at the bottom of Figure 4 (scheme (a)). The solid and dashed lines denote levels mainly associated with the l-and u-manifolds, respectively. As expected, the energies of the excitonic levels are very similar to those of Sauer et al. [4] As shown in Refs. $[4,5]$, the structure of the B850 ring dictates that the lowest energy $\mathrm{E}_{1}$ level carried almost all of the absorption intensity. This is why the lowest $E_{1}$ level is placed at the maximum of the B850 absorption band in Figure 4. Wu and Small pointed out that levels of $E_{2}, E_{3}$ and $E_{4}$ symmetry are forbidden [40]. The A levels and upper $\mathrm{E}_{1}$ level, although symmetry allowed, are highly forbidden because of the structural arrangement of the $Q_{y}$-transition dipoles. The lowest $\mathrm{A}$ level was predicated to carry $<1 \%$ of the absorption intensity of the $\mathrm{B} 850$ band in the absence of energy disorder [4,5]. An important benchmark for excitonic calculations is the splitting, $\Delta \mathrm{E}$, between the lowest $\mathrm{A}$ and $\mathrm{E}_{1}$ levels. The calculations of Sauer et al. [4] and Alden et al. [5] led to a $\Delta E$-value close to $100 \mathrm{~cm}^{-1}$. The value in energy level scheme (a) of Figure 4 is $110 \mathrm{~cm}^{-1}$. The experimental value, as determined at $4 \mathrm{~K}$ by ZPH action 
spectroscopy, is $200 \mathrm{~cm}^{-1}[27,28]$. Furthermore, the percentage contribution of the A level (often referred to as B870) to the intensity of the B850 band was found to be $\sim 3 \%$.

Energy disorder, whether diagonal or off-diagonal, couples the $A$ and $E_{1}$ levels, thereby increasing $\Delta \mathrm{E}$ and the absorption intensity of the $\mathrm{A}$ level by intensity borrowing from the $\mathrm{E}_{1}$ level. However, energy disorder of sufficient strength to yield $\Delta \mathrm{E}=200 \mathrm{~cm}^{-1}$ resulted in far too high an absorption intensity for the B870 band [23]: Wu et al. [23] concluded that the nearest monomer-monomer coupling energy for adjacent dimers at $4 \mathrm{~K}$ is substantially greater than that calculated in Ref. [4] using the room temperature structure. This was strongly supported by the temperature dependence of the B850 absorption band [28] which showed that LH2 complex undergoes a structural change at $150 \mathrm{~K}$ (also observed for $R b$. sphaeroides [28]). Theoretical analysis of the thermal broadening data, which are characteristic of downward inter-exciton level relaxation, led to the conclusion that the above coupling for the low temperature structure is $\sim 35 \%$ stronger than that of the high temperature structure. It was suggested that this increase is primarily associated with electron-exchange coupling.

The $35 \%$ increase in coupling leads to $V_{u}=170 \mathrm{~cm}^{-1}, V_{1}=-270 \mathrm{~cm}^{-1}$, and $V_{u t}=$ $200 \mathrm{~cm}^{-1}$. The resulting excitonic energies obtained with $\mathrm{e}_{\mathrm{u}}-\mathrm{e}_{\mathrm{l}}=600 \mathrm{~cm}^{-1}$ are shown in scheme (b) in Figure 4. Comparing schemes (a) and (b), one sees that for (a) the five highest energy levels are symmetrically disposed about the B800 band while in (b) they lie to the blue of the $\mathrm{B} 800$ band maximum. The $\Delta \mathrm{E}$-value for (b) is $150 \mathrm{~cm}^{-1}$. It is likely that the structural change at $150 \mathrm{~K}$ also increases the monomer-monomer coupling of the basic dimer. However, the two monomers of that dimer are symmetry inequivalent; their site excitation energies could differ by $\sim 200 \mathrm{~cm}^{-1}$ [5]. This being the case, the effect of the 
structural change on the energies of the $u$ and 1 dimer levels would be diluted. The result of increasing $e_{\mathrm{u}}-\mathrm{e}_{\mathrm{l}}$ to $690 \mathrm{~cm}^{-1}$ (a $15 \%$ increase) is shown as scheme (c) in Figure 4. As expected, this increase has only a small effect on the splittings between the $A, E_{1}$ and $E_{2}$ levels associated with the B850 band. The main effects are to shift the five highest energy levels further to the blue and change the splittings between them.

Schemes (b) and (c) of Figure 4 are consistent with the exciton levels that stem from the $\mathrm{u}$-dimer level lying to higher energy of the $\mathrm{B} 800$ band maximum. This is also the case for the $E_{4}$ level associated with 1-dimer level. We believe that the values of $170,-270$ and $200 \mathrm{~cm}^{-1}$ for $\mathrm{V}_{\mathrm{u}}, \mathrm{V}_{\mathrm{l}}$ and $\mathrm{V}_{\mathrm{ul}}$, respectively, are reasonable since, when diagonal energy disorder is taken into account, a $\Delta \mathrm{E}$ value of $\sim 200 \mathrm{~cm}^{-1}$ and $\sim 3 \%$ contribution from $\mathrm{B} 870$ to the B850 absorption band was obtained [23]. The value of $e_{u}-e_{1}$ is more uncertain. There have been many calculations of the excitonic structure of the $\mathrm{B} 850$ ring of Rps. acidophila. All are based on the room temperature structure of $\mathrm{LH} 2$ and, thus, do not take into account the structural change at $150 \mathrm{~K}$. The reader is referred to Linnanto et al. [41] for discussion of the range of values obtained for the intradimer monomer-monomer coupling and nearest monomer-monomer coupling between adjacent dimers and the methods and assumptions used. The variations in these couplings are quite significant, as are the widths of the B850 exciton manifold they lead to. Suffice to say that exciton manifolds (b) and (c) in Figure 4 cannot be argued at this time to be unreasonable. We note that Koolhaas et al. [30] recently concluded that the nearest neighbor coupling in the B850 ring of Rbs. sphaeroides at $77 \mathrm{~K}$ is $\sim 300 \mathrm{~cm}^{-1}$, a value obtained from theoretical analysis of the CD spectrum of an upper B850 exciton level located on the blue side of the B800 band. 
It follows from level schemes (b) and (c) in Figure 4 that mixing of the upper B850 exciton levels should be stronger with B800 molecules that absorb on the high energy side of the $\mathrm{B} 800$ band. (The strongest coupling between $\mathrm{B} 800$ and $\mathrm{B} 850$ molecules is $\sim 30 \mathrm{~cm}^{-1}$ [4].) The resulting mixed B800-B850 states may be responsible for the tailing of the B800 band on its high energy side. The Franck-Condon factors for low frequency intramolecular BChl $a$ modes [42] are too small to account for the tailing, results not shown.

The interpretation that has mixed $\mathrm{B} 800-\mathrm{B} 850$ states figuring importantly in the additional B800 decay channel is plausible since mixing of the upper B850 levels with nearby $\mathrm{B} 800$ levels in intact $\mathrm{LH} 2$, with its nine $\mathrm{B} 800$ molecules, can be expected to be significantly stronger than in LH2 complexes containing only one B800 molecule. (Obviously, the B800 absorption intensity from which the B850 levels can steal intensity is nine times greater in native LH2.) Excitonic calculations that take into account energy disorder, which determine the extent to which the B850 levels are delocalized, are required to assess how much stronger the mixing in native LH2 is. To a good approximation, one could assume that $\mathrm{B} 800$ excitations are localized on single B800 molecules and that removal of B800 molecules has no effect on the structure of the B850 ring (vide infra). Such calculations are beyond the scope of this paper. It is, however, instructive to consider a B850 level localized on a dimèr that interacts with a complex containing only one B 800 molecule. The probability that the dimer would be positioned to make the closest possible contact with that B800 molecule is $1 / 9$ whereas it is 1 for intact LH2. Of course, dimers not in closest proximity to the $B 800$ molecule would need to be taken into account. It should be noted the situation for $\mathrm{B} 800 \rightarrow \mathrm{B} 850$ transfer is different. Here, in the first approximation, one expects 
that the rates for intact $\mathrm{LH} 2$ and $\mathrm{B} 800$-deficient $\mathrm{LH} 2$ should be similar. The holewidth data are consistent with this.

There are experimental results that provide some support for mixed B800-B850 states located on the high energy side of the B800 band. First, the pump-probe spectra of ref 16 obtained with excitation on the blue side of the $B 800$ band $(T=19 K)$ revealed an essentially prompt $(\sim 30 \mathrm{fs})$ excited state absorption of $\mathrm{B} 850$, the expected behavior for excitation of a mixed B80-B850 state. Second, the magnitude of the permanent dipole moment change for the $\mathrm{S}_{0} \rightarrow B 800^{*}$ transition, as determined by Stark-hole burning spectroscopy, was observed to increase by $10 \%$ as the burn frequency was tuned from the red edge of the B800 band to its maximum [43]. Such behavior is opposite to that routinely observed for an inhomogeneously broadened absorption band of a "pure" state. We plan to extend the Stark measurements to the blue edge of the B800 band as well as perform high pressure-hole burning experiments with burn frequencies that range from the red to blue edges of the $\mathrm{B} 800$ band since the linear pressure shift rate for the $\mathrm{B} 850$ band is a factor of $\sim 4$ greater than that of the B800 band [28]. The results of such experiments should allow for further testing of the above proposal. Although other mechanisms we have not considered may be possible, the above mechanism, given our present knowledge, seems the most reasonable one.

To conclude this subsection we consider possible relaxation pathways for the mixed B800-B850 states. Downward relaxation between them which culminates in population of levels that contribute to the low energy side of the B800 band and which are mainly "B800" in character is one. This pathway is in accord with the femtosecond pump-probe spectra reported in [16] which were obtained by pumping on the high energy side of the B800 band 
and probing at lower energies within the $\mathrm{B} 800$ band. The second pathway would be downward relaxation to levels that lie lower in energy than the B800 band, i.e. the lowest five levels in schemes (b) and (c) of Figure 4. Such a pathway is also suggested by the results in Ref. [16] which show that pumping on the high energy side of the B800 band results in prompt excited state absorption of the B850 ring. Detailed excitonic calculations that take into account energy disorder and correctly deal with the vibrational and phononic structure associated with the B850 exciton levels are required to assess the relative importance of the two pathways. It should be noted, in the case of the B850 ring, that the vibronic structure problem is difficult because one is in the strong coupling limit where the width of the exciton manifold is $\gtrsim$ intramolecular vibrational frequencies [44]. In calculations performed thus far the opposite limit was assumed, see, e.g., Refs. [34,35].

B850 excitonic structure in B800-deficient LH2. As mentioned, the B850 band of B800-deficient LH2 is slightly red-shifted $(1.2 \mathrm{~nm})$ relative to that of intact LH2 and somewhat broader ( 235 vs. $210 \mathrm{~cm}^{-1}$ ). One cannot be sure that these rather small differences are due to removal of $\mathrm{B} 800$ molecules since differences of this magnitude have been observed for different samples of intact LH2. For example, a B850 bandwidth of $200 \mathrm{~cm}^{-1}$ was reported in [28] for an intact LH2 sample prepared by the same isolation and purification procedure used in this work. Nevertheless, the spectra of Figure 1 indicate that removal of B800 molecules has a weak effect on the B850 ring. This is supported by the observation that the thermal broadening and shifting of the $\mathrm{B} 850$ band of B800-deficient LH2 is identical to those of intact LH2 [28] (results not shown). As discussed in detail in Ref. [28], the thermal broadening is characteristic of downward inter-exciton level relaxation. The thermal broadening of the B800 band is entirely different and characteristic of that observed for 
monomeric optical transitions. Additional support is provided by the ZPH action spectrum of the lowest A exciton level (B870) of B800-deficient LH2, Figure 5. Here, the solid circles are the $\triangle \mathrm{OD}$ values of $\mathrm{ZPH}$ burned at different wavelengths. Zero-phonon hole action spectroscopy, first introduced in Ref. [45], has proven to be a powerful technique for locating and characterizing the lowest exciton level of photosynthetic complexes [46]. The solid curve through the data points is a Gaussian fit that yields a fwhm of $125 \mathrm{~cm}^{-1}$, within experimental uncertainty the same as the value reported in [27]. To a good approximation the Gaussian curve is the site distribution function (SDF) for $\mathrm{B} 870$. However, $\Delta \mathrm{E}$ (displacement between the maxima of the B850 absorption band and the action spectrum) is $245 \mathrm{~cm}^{-1}, 45 \mathrm{~cm}^{-1}$ larger than the value reported in [27]. We remind the reader that $\Delta \mathrm{E}$ is the apparent energy gap between the $A$ level (B870) and the strongly allowed $E_{1}$ level and that in order to obtain a "true" value one needs to take into account the distribution of peak absorption cross-sections for $\mathrm{B} 870$ of different single complexes. This distribution is a consequence of structural heterogeneity. The result is that the apparent value of $\Delta \mathrm{E}$ is larger than the true value [23]. Although the above discrepancy of $45 \mathrm{~cm}^{-1}$ may be due to an increase in coupling between B850 molecules in B800-deficient LH2, we think it more likely that it is mainly a consequence of greater energy disorder in B800-deficient LH2. One expects that increasing energy disorder (diagonal or off-diagonal) should be reflected by an increase in width of the B850 absorption band. Increasing energy disorder also leads to an increase in $\Delta \mathrm{E}$ as first shown in Refs. [5,40]. As discussed in Ref. [23], $\Delta \mathrm{E}$ for both $R b$. sphaeroides and Rps. acidophila has decreased over the years as the quality of isolated LH2 complexes has improved, i.e. as the widths of the B800 and B850 bands have decreased. For example, the $\Delta \mathrm{E}$ values reported for $R b$. sphaeroides samples that exhibited $\mathrm{B} 850$ 
bandwidths of 280 and $230 \mathrm{~cm}^{-1}$ are 250 and $185 \mathrm{~cm}^{-1}$, respectively. Returning to Figure 5, $\Delta \mathrm{E}=245 \mathrm{~cm}^{-1}$ and the width of the $\mathrm{B} 850$ band is $245 \mathrm{~cm}^{-1}$. The value of $\Delta \mathrm{E}=200 \mathrm{~cm}^{-1}$ reported in Ref. [23] was for a sample exhibiting a B850 bandwidth of $200 \mathrm{~cm}^{-1}$. We conclude, therefore, that almost complete removal of the B800 molecules does not significantly affect the excitonic structure of the B850 ring.

\section{Conclusions and Final Remarks}

The primary motivation for this work was to gain new insight on the nature of the additional B800 decay channel which is observed upon excitation on the high energy side of the B800 absorption band. To this end, NPHB experiments were performed on LH2 complexes of Rps. acidophila (strain 10050) that contain only one $\mathrm{B} 800 \mathrm{BChl} a$ molecule. The key finding is that the zero-phonon holewidths associated with the B800 band of B800deficient LH2 are independent of the location of the burn frequency within that band (Figure 2), in sharp contrast with intact LH2 (Figure 3). It was determined, on the basis of the temperature dependence of the B850 absorption band and zero-phonon hole action spectrum (Figure 5) of the lowest exciton level of A symmetry (B870), that the removal of B800 molecules has essentially no effect on the excitonic structure of the B850 ring. This, together with the above independence of the holewidths, led us to eliminate mechanisms (i) and (ii) (see Abstract) for the additional decay channel. It could be concluded, therefore, that this channel must involve B800 intraband energy transfer. It was argued, however, that the holewidth data in Figure 3 for intact LH2 are inconsistent with downward B800-B800 energy transfer involving only the B800 molecules (mechanism (iii)). This followed from consideration of the spectral density that enters into the rate expression for energy transfer. 
We have proposed that the additional decay channel is due to mixed B800-B850 levels that contribute significantly to the high energy side of the B800 absorption band. Results from previous studies that provide some support for this model were presented. However, additional experiments are required to test this model. They include Stark- and high pressure-hole burning experiments with burn frequencies that span the entire B800 absorption band. Such experiments are planned. More detailed ultrafast pump-probe experiments which test the notion that the mixed B800-B850 states could decay by two pathways should also be informative. The two suggested pathways are relaxation to B800 levels which are mainly "B800" in character and dominate the absorption on the low energy side of the B800 band and relaxation to lower energy levels mainly associated with the B850 ring. Excitonic structure and energy transfer calculations which account for energy disorder and incorporate appropriate spectral densities are also required. In this regard, we suggest that the effective Hamiltonian defined by Eqs. 2-4 with the parameter values used to generate exciton levels schemes (b) and (c) in Figure 4 would be good starting points. Concerning the determination of an acceptable level of energy disorder in the low temperature limit, we have reemphasized $[27,28]$ the importance of the splitting $(\Delta \mathrm{E})$ between the lowest $\mathrm{E}_{1}$ and $\mathrm{A}$ (B870) measured by ZPH hole action spectroscopy and the $\sim 3 \%$ contribution of $\mathrm{B} 870$ to the $\mathrm{B} 850$ absorption band.

Finally, it should be emphasized that the residual holewidths given in Figure 3 determine the decay times of the additional decay channel $\left(10 \mathrm{~cm}^{-1}=1 \mathrm{ps}\right)$. 


\section{Acknowledgements}

Research at the Ames Laboratory was supported by the Division of Chemical Sciences, Office of Basic Energy Sciences, U.S. Department of Energy. Ames Laboratory is operated for USDOE by Iowa State University under Contract W-7405-Eng-82. Research at the University of Glasgow was supported by the Gatsby Trust and the BBSRC. G. J. Small would like to thank R. S. Silbey (MIT) and X. Song (ISU) for helpful discussions.

\section{References}

1. van Grondelle, R.; Dekku, J. P.; Gillbro, T.; Sundström, V. Biochim. Biophys. Acta 1994, 1187, 1; Pullerits, T.; Sundström, V. Acc. Chem. Res. 1996, 29, 381; Sundström, V.; van Grondelle, R. In Anoxygenic Photosynthetic Bacteria; Blankenship, R. E.; Madigan, M. T.; Baller, C. E., Eds.; Kluwer Academic Publishers: Dordrecht, 1995, p. 349.

2. Freer, A. A.; Prince, S. M.; Sauer, K.; Papiz, M. Z.; Hawthornewaite-Lawless, A. M.; McDermott, G.; Cogdell, R. J.; Isaacs, N. W. Structure, 1996, 4, 449.

3. Koepke, J.; Xu, X.; Muenke, C.; Schulten, K.; Michl, H. Structure, 1996, 4, 561.

4. Sauer, K.; Cogdell, R. J.; Prince, S. M.; Freer, A. A.; Isaacs, N. W.; Scheer, H. Photochem. Photobiol. 1996, 64, 564.

5. Alden, R. G.; Johnson, E.; Nagarajan, V.; Parson, W. W.; Law, C.; Cogdell, R. J. $J$. Phys. Chem. B 1997, 101, 4667.

6. Karrasch, S.; Bullough, P. A.; Ghosh, R. EMBO J. 1995, 14, 631.

7. Sundström, V.; Pullerits, T.; van Grondelle, R. J. Phys. Chem. B 1999, 103, 2327.

8. Book, L. D.; Ostatin, A. E.; Ponomarenko, N.; Norris, J. R.; Scherer, N. F. J. Phys. Chem. B 2000, 104, 8295 .

9. Shreve, A. P.; Trautman, J. K.; Frank, H. A.; Owens, T. J.; Albrecht, A. C. Biochim. Biophys. Acta 1991, 1058, 280.

10. Hess, S.; Felschtein, F.; Babin, A.; Nurgaleev, I.; Pullerits, T.; Sergeev, A.; Sundström, V. Chem. Phys. Lett. 1993, 216, 247. 
11. Pullerits, T.; Hess, S.; Herek, J. L.; Sundström, V. J. Phys. Chem. 1997, 101, 10560.

12. Joo, T.; Jia, Y.; Yu, J.-Y.; Jonas, D. M.; Fleming, G. R. J. Phys. Chem. 1996, 100, 2399.

13. Jiminez, R., Dikshit, S. N.; Bradforth, S. E.; Fleming, G. R. J. Phys. Chem. 1996, 100,6825 .

14. Ma, Y.-Z.; Cogdell, R. J.; Gillbro, T. J. Phys. Chem. B 1997, 101, 1087.

15. Ma, Y.-Z.; Cogdell, R. J.; Gillbro, T. J. Phys. Chem. B 1998, $102,881$.

16. Wu, H.-M.; Savikhin, S.; Reddy, N. R. S.; Jankowiak R.; Cogdell, R. J.; Struve, W. S.; Small, G. J. J. Phys. Chem. 1996, 100, 12022.

17. van der Laan, H.; Schmidt, Th.; Visschers, R. W.; Visschers, K. J.; van Grondelle, R.; Völker, S. Chem. Phys. Lett. 1990, 170, 231.

18. Reddy, N. R. S.; Small, G. J.; Seibert, M.; Picorel, R. Chem. Phys. Lett. 1991, 181, 391.

19. Reddy, N. R. S.; Small, G. J. J. Chem. Phys. 1991, 94, 7545.

20. De Caro, C., Visschers, R. W.; van Grondelle, R.; Völker, S. J. Phys. Chem., 98, 10584.

21. Reddy, N. R. S.; Cogdell, R. J.; Zhao, L.; Small, G. J. J. Photochem. Photobiol. $1993,57,35$.

22. van Oijen, A. M.; Ketelaars, M.; Köhler, J.; Aartsma, T. J.; Schmidt, J. Biophysical J. $2000,78,1570$.

23. Wu, H.-M.; Rätsep, M.; Lee, I.-J.; Cogdell, R. J.; Small, G. J. J. Phys. Chem. B 1997, 101,7654 .

24. Cogdell, R. J.; Hawthornewaite, A. M. In The Photosynthetic Reaction Center; Deisenhofer, J., Norris, J. R., Eds.; Academic Press, San Diego, 1993; Vol. 1, p. 23.

25. Gall, G.; Fraser, N. J.; Bellissent-Funel, M.-C.; Scheer, H.; Robert, B.; Cogdell, R. J. FEBS Lett. 1999, 449, 269; Fraser, N. J. Ph.D. Thesis 1999, University of Glasgow, Glasgow.

26. Chang, H.-C.; Jankowiak, R.; Reddy, N. R. S.; Small, G. J. Chem. Phys. 1995, 197, 307. 
27. Wu, H.-M.; Reddy. N. R. S.; Small, G. J. J. Phys. Chem. B 1997, 101, 651.

28. Wu, H.-M.; Rätsep, M.; Jankowiak, R.; Cogdell, R. J.; Small, G. J. J. Phys. Chem. B $1997,101,7641$.

29. Herck, J. L.; Fraser, N. J.; Pullerits, T.; Martinsson, P.; Polivka, T.; Scheer, H.;

Cogdell, R. J.; Sundström, V. Biophysical J. 2000, 78, 2590.

30. Koolhaas, M. H. C.; Frese, R. N.; Fowler, G. J. S.; Bibby, T. S.; Georgakopoulu, S.; van der Zwan, G.; Hunter, C. N.; van Grondelle, R. Biochemistry 1998, 37, 4693.

31. Reddy, N. R. S.; Wu, H.-M.; Jankowiak, R.; Picorel, R.; Cogdell, R. J.; Small, G. J. Photosyn. Res. 1996, 48, 277.

32. Fowler, G. J. S.; Hess, S.; Pullerits, T.; Sundström, V.; Hunter, C. N. Biochemistry $1997,36,11282$.

33. Kühn, O; Sundström, V. J. Phys. Chem. B 1997, 101, 3432.

34. Mukai, K.; Abe, S.; Sumi, H. J. Phys. Chem. B 1999, 103, 6096.

35. Scholes, G. D.; Fleming, G. R. J. Phys. Chem. B 2000, 104, 1854.

36. Let $j=0,1, \ldots$ label the $B 800$ molecules and $V_{01}^{2}$ be the square of the nearest neighbor coupling. From the LH2 structure of Rps. acidophila it follows that $\mathrm{V}_{02}^{2}, \mathrm{~V}_{03}^{2}$, and $\mathrm{V}_{04}^{2}$ equal $0.037,0.01$ and 0.005 in units of $\mathrm{V}_{01}^{2}$.

37. Kolaczkowski, S. V.; Hayes, J. M.; Small, G. J. J. Phys. Chem. 1994, 98, 13418.

38. See Ref. [16] and references therein.

39. Wu, H.-M.; Rätsep, M.; Jankowiak, R.; Cogdell, R. J.; Small, G. J. J. Phys. Chem. B 1998, $102,4023$.

40. Wu., H.-M.; Small, G. J. Chem. Phys. 1997, 218, 225.

41. See Linnanto, J.; Korppi-Tommola, J. E. I.; Helenius, V. M. J. Phys. Chem. B 1999, 103,8739 .

42. Wendling, M.; Pullerits, T.; Przjalgowski, M. A.; Vulto, S. I. E.; Aartsma, T. J.; van Grondelle, R.; van Amerongen, H. J. Phys. Chem. B 2000, 104, 5825.

43. Rätsep, M.; Wu, H.-M.; Hayes, J. M.; Small, G. J. Spectrochim. Acta A 1998, 54, 1279 . 
44. See Lathrop, E. J. P.; Friesner, R. A. J. Phys. Chem. 1994, 98, 3050 and teferences therein.

45. Reddy, N. R. S., Picorel, R.; Small, G. J. J. Phys. Chem. 1992, 96, 6458.

46. See, for example, den Hartog, F. T. H.; Dekker, J. P.; van Grondelle, R.; Völker, S. $J$. Phys. Chem. B 1998, 102, 11007. Jankowiak, R.; Rätsep, M.; Picorel, R.; Seibert, M.; Small, G. J. J. Phys. Chem. B 1999, 103, 9759. den Hartog, F. T. H.; Vacha, F.; Lock, A. J.; Barber, J.; Dekker, J. P.; Völker, S. J. Phys. Chem. B 1998, 102, 9174. Fetisova, Z. G.; Mauring, K. FEBS Lett. 1993, 323, 159. Psencik, J.; Polivka, T.; Nemec, P.; Dian, J.; Kudma, J.; Maly, P.; Hala, J. J. Phys. Chem. A 1998, 102, 4392. 


\section{Figure Captions}

Figure 1. The $4.2 \mathrm{~K}$ absorption spectra of intact (a) and B800-deficient LH2 (b) normalized to equal peak intensities of the B850 band. Inset: spectrum (b) is an expanded view of the $800 \mathrm{~nm}$ region of $\mathrm{B} 800$-deficient $\mathrm{LH} 2$; spectrum (c) is that of the same sample after storage in the dark at $0^{\circ} \mathrm{C}$ for one week.

Figure 2. Widths of zero-phonon holes burned into the B800 band with different burn frequencies at $4.2 \mathrm{~K}$ (inset). The burn intensity and time were $\sim 120 \mathrm{~mW} / \mathrm{cm}^{2}$ and $100 \mathrm{~s}$. The fractional hole depths were $0.02-0.03$. The uncertainty in the widths is $\pm 0.3 \mathrm{~cm}^{-1}$. Thus, the holewidth is independent of burn frequency. The average of the holewidths is $3.2 \pm 0.3 \mathrm{~cm}^{-1}$ which corresponds to a $\mathrm{B} 800 \rightarrow \mathrm{B} 850$ transfer time of $3.2 \mathrm{ps}$ (see text for discussion). For convenience the absorption spectrum from Figure 1 is shown.

Figure 3. Widths of zero-phonon holes burned into the $\mathrm{B} 800$ band of intact LH2. The diamond data points are from this study. The burn intensity and time were $\sim 120$ $\mathrm{mW} / \mathrm{cm}^{2}$ and $100 \mathrm{ps}$. Fractional hole depths did not exceed 0.03 . The solid circle data point is from Ref. [16] and corresponds to a holewidth of $26 \mathrm{~cm}^{-1}$. Importantly, it falls on the straight line defined by the four diamond data points. The inset shows the SDF of the B800 molecules (width $=120 \mathrm{~cm}^{-1}$ ) and a sigmoidal curve calculated with Eq. 1 with $\mathrm{D}\left(\omega_{\mathrm{B}}-\omega\right)$ a constant. See text for discussion.

Figure 4. Exciton levels of the B850 ring calculated with the Hamiltonian defined by Eqs. 2-4. The values used for $e_{u}-e_{l}, V_{l}, V_{u}$ and $U_{u l}$ are, respectively (in the unit of $\mathrm{cm}^{-1}$ ): (a) $600,-200,100,130$; (b) 600,-270, 170, 200; and (c) 690,-200, 100, 130. The solid and dashed vertical lines denote exciton levels mainly associated with the 1 - and u-manifolds (see text). The asterisk indicates closely spaced levels of $E_{2}$ and $E_{4}$ symmetry belonging to the 1- an u-manifolds, respectively. The $X$ indicates closely spaced $E_{3}$ and $E_{4}$ levels belonging to the 1- and u-manifolds. 
Figure 5. The zero-phonon hole action spectrum of the lowest energy A level of the B850 ring (B870) for B800-dificient $\mathrm{LH} 2$. The circles are the $\Delta$ absorbance changes for holes burned at different frequencies with a constant burn intensity of $50 \mathrm{~mW} / \mathrm{cm}^{2}$ and burn time of $30 \mathrm{~s}$. The maximum fractional hole depth was 0.06 . For ease of discussion the $\mathrm{B} 850$ absorption band is also shown. 


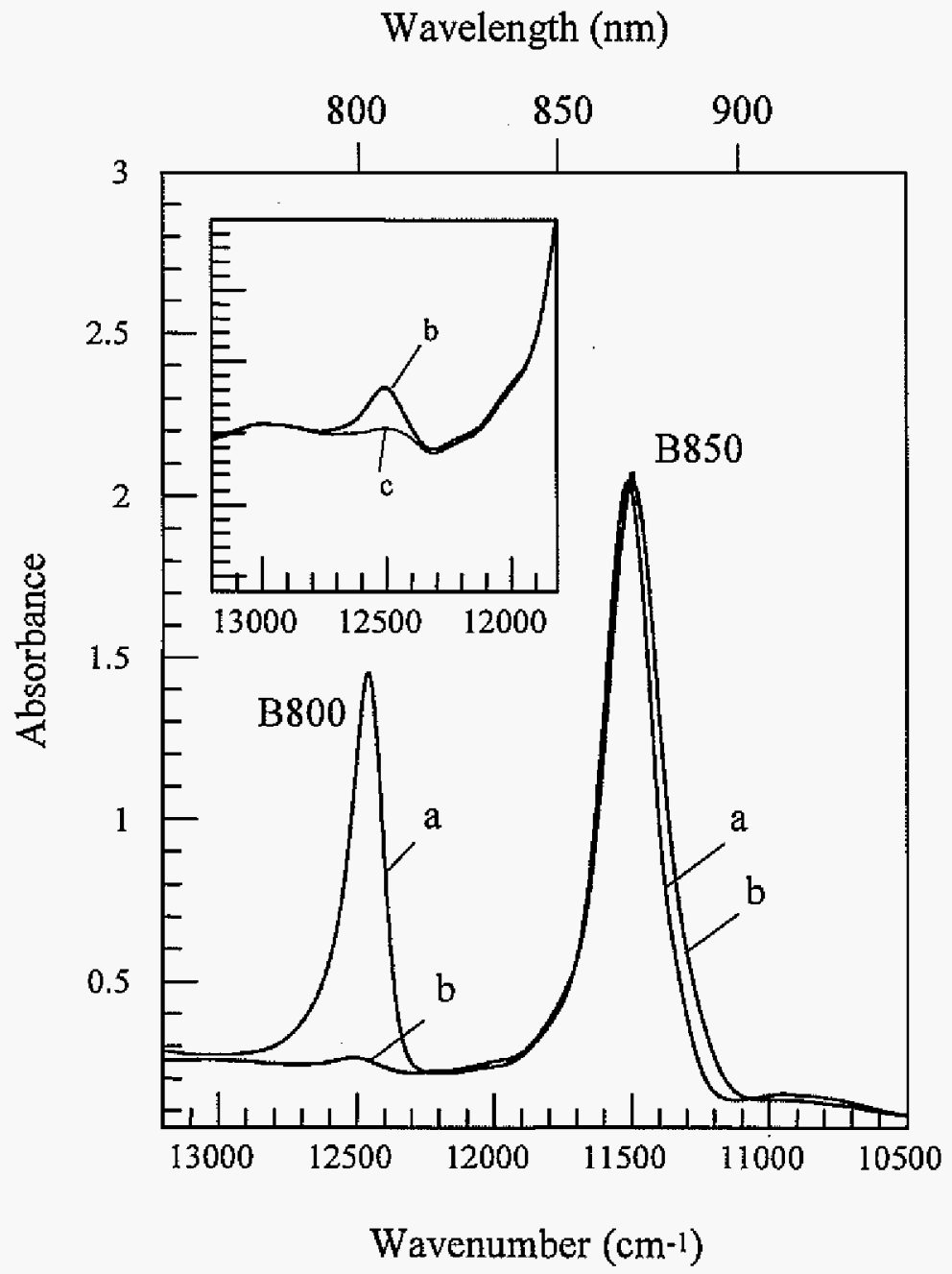

Figure 1. 


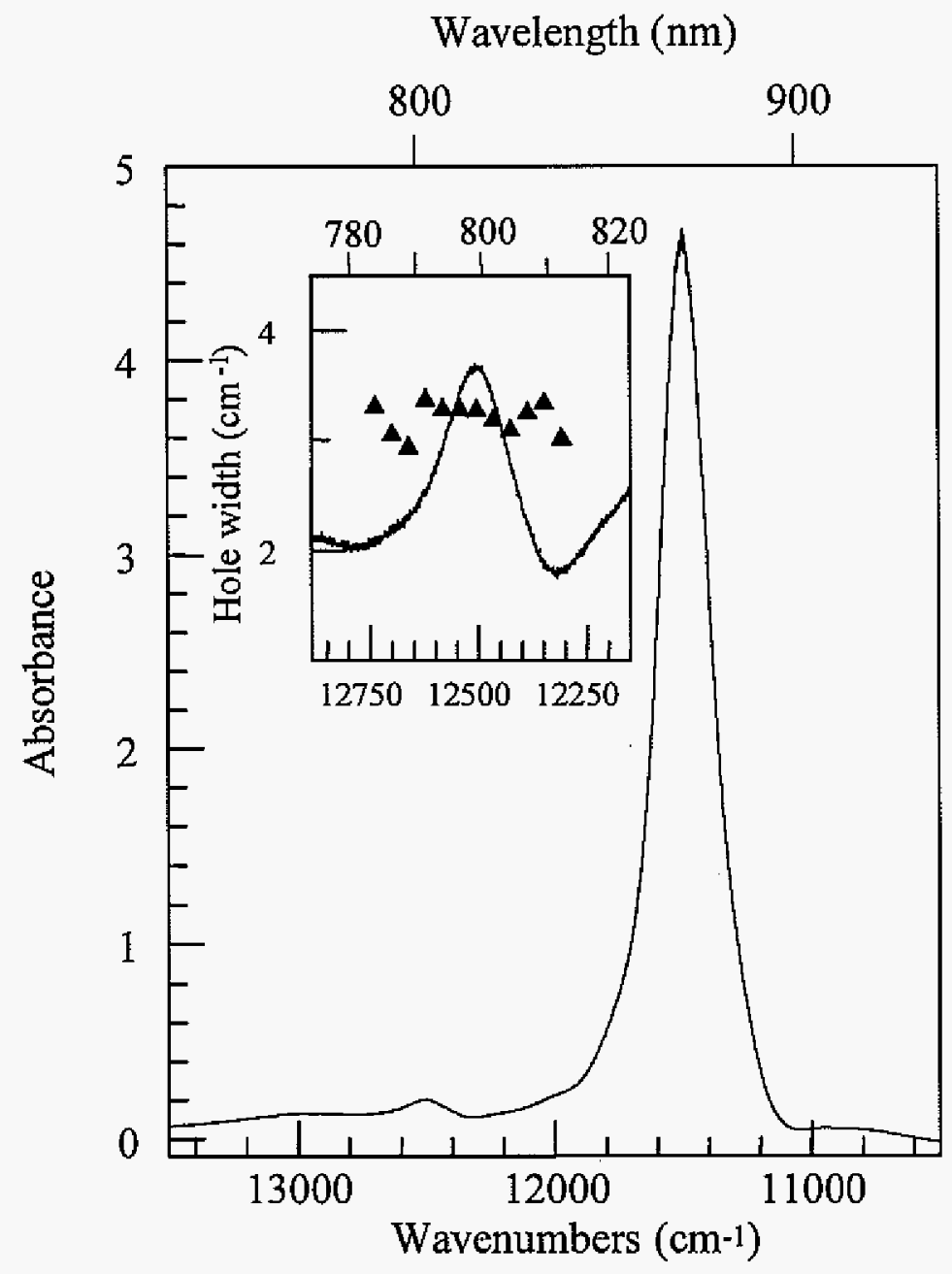

Figure 2. 


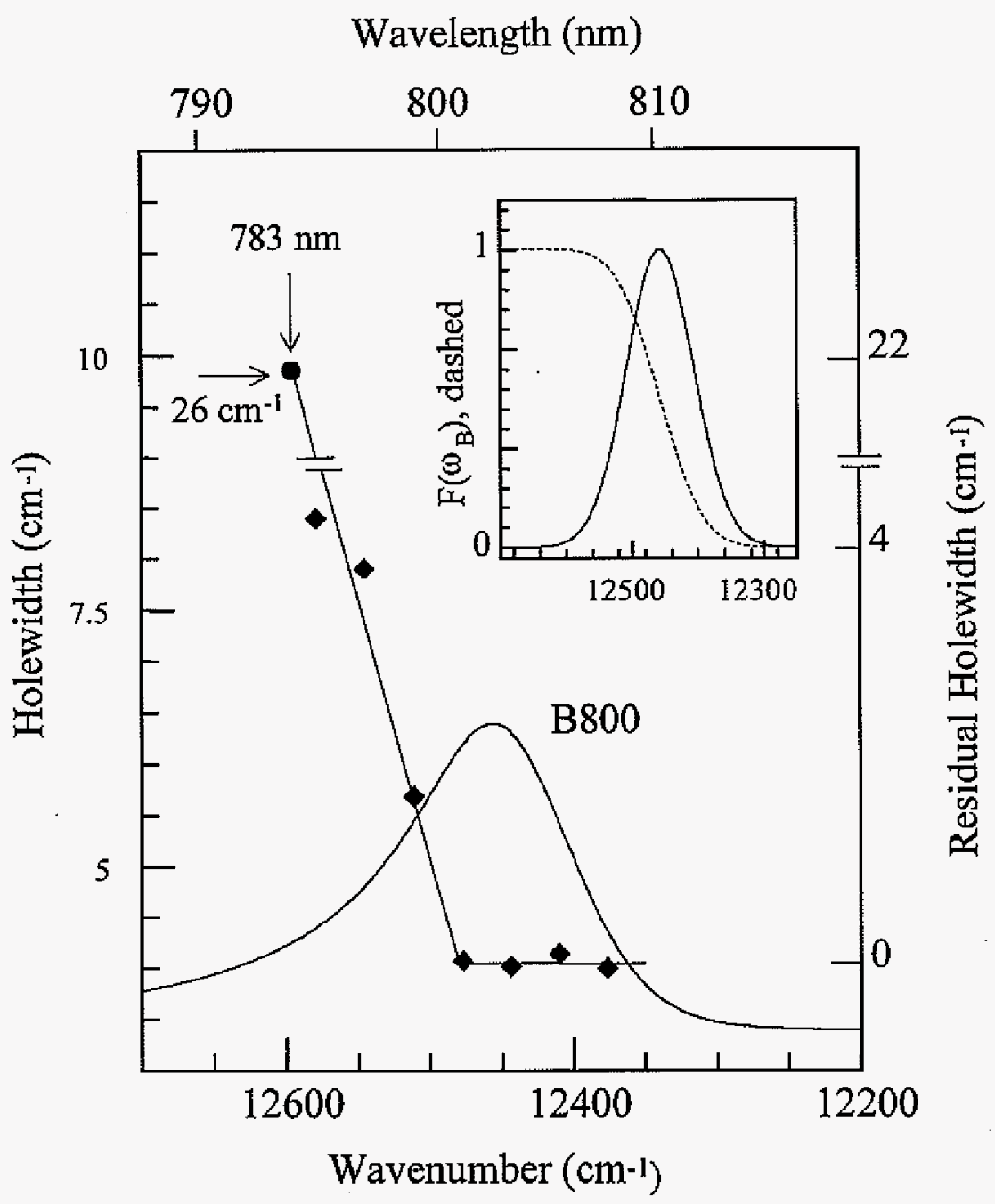

Figure 3. 


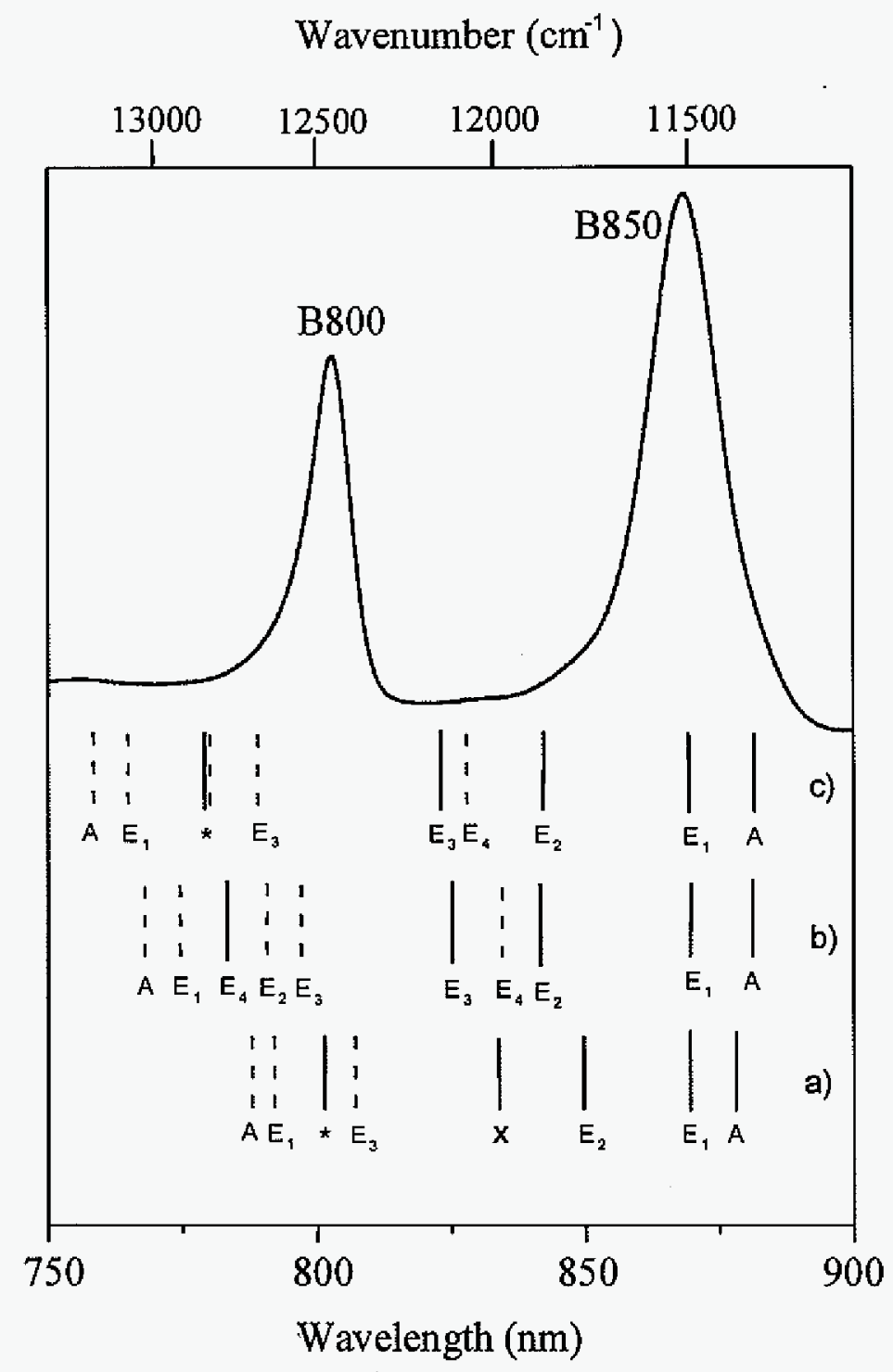

Figure 4. 


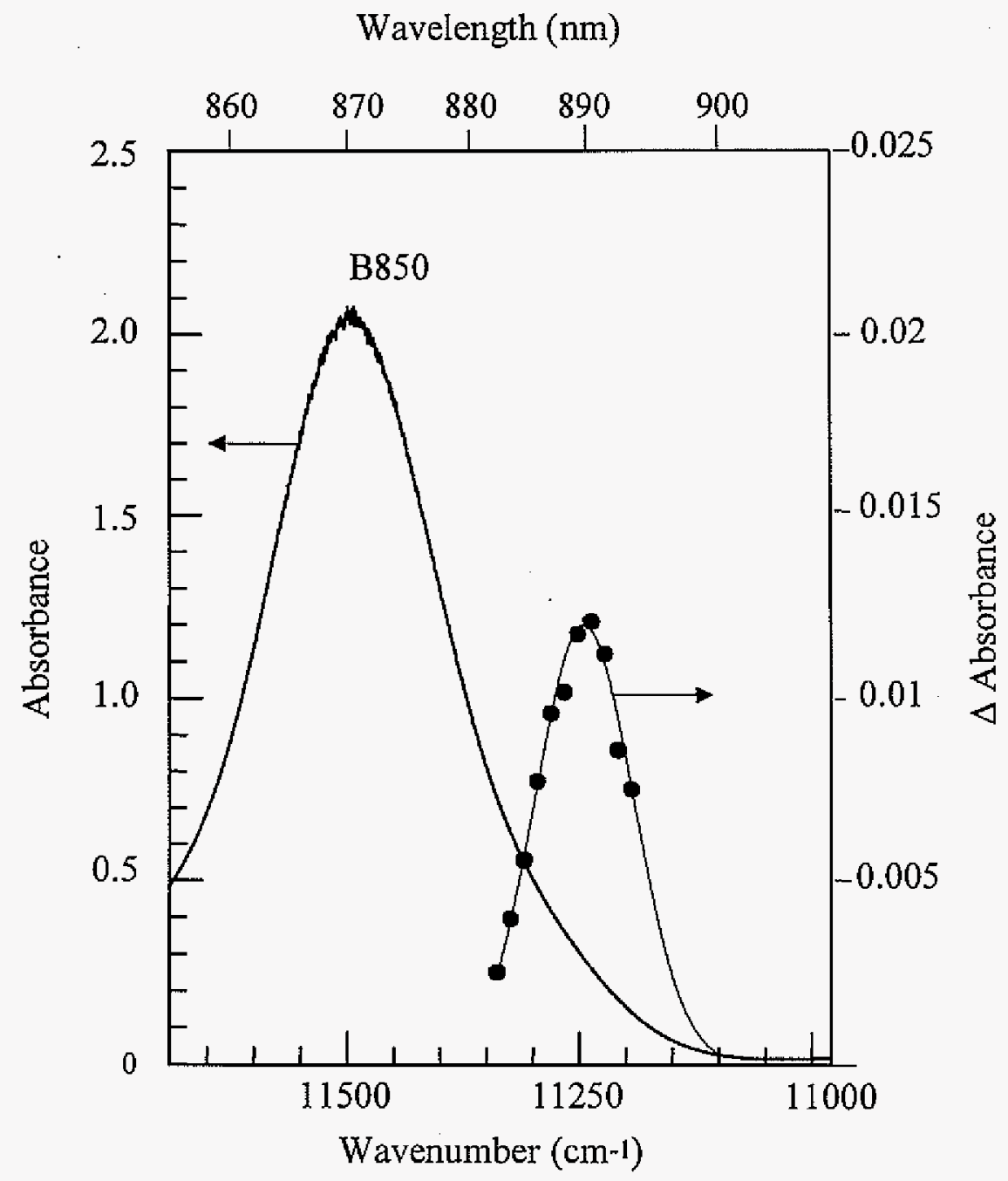

Figure 5. 


\section{CHAPTER 5. GENERAL CONCLUSIONS}

Nonphotochemical hole-burning (NPHB) spectroscopy was used in this work to study bacterial antenna complexes. The research focused on the $\mathrm{Q}_{\mathrm{y}}$-state electronic structures and energy transfer dynamics of these systems. Theoretical models were used to simulate the data obtained and determine how well the assumptions made in the models successfully describe the systems.

The lowest energy absorption band at $825 \mathrm{~nm}$ of FMO complex from $P$. aestuarii, which consists of three states, was burned nonphotochemically and its low energy satellite holes were analyzed to determine the excitation energy transfer (EET) kinetics for the contribution of two higher energy states to the $825 \mathrm{~nm}$ band. The satellite holes at $36,48,72$, 120 and $165 \mathrm{~cm}^{-1}$ were concluded, based on simulations, to be pseudo-phonon sideband holes (PSBH) and these phonons were considered due to $\mathrm{BChl} a$ intramolecular modes. In addition, the temperature dependencies of zero-phonon hole (ZPH) widths burned in the 825 $\mathrm{nm}$ band at $\lambda_{\mathrm{B}}=822.6,824.6$ and $826.6 \mathrm{~nm}$ showed similarity to those of $\mathrm{Cb}$. tepidum and these three wavelengths were close to the absorption maxima of the three states. Additional energy transfer calculations that account the effects of energy disorder into the electronphonon coupling parameters were suggested for future study.

NPHB on LH2 complexes of Rps. acidophila (strain 10050) that contain only one $\mathrm{B} 800 \mathrm{BChl} a$ molecule, in contrast with intact $\mathrm{LH} 2$, were performed to determine an additional B800 decay channel. ZPH action spectrum of the lowest exciton level (A symmetry) and the temperature dependency of the B850 absorption band found a deficiency of B800 molecules does not affect on the excitonic structure of the B850 ring. The above 
results and another finding, which showed ZPH widths associated with the B800 band (of B800-deficient LH2) are independent of $\lambda_{B}$ within the band, concluded that the decay channel is due to mixed $\mathrm{B} 800-\mathrm{B} 850$ levels, which may be a major contribution to the high energy side of the B800 absorption band. The model established the necessity for additional experiments such as Stark- and high pressure-hole-burning experiments and/or ultrafast pump-probe experiments, as well as further excitonic level calculations taking account of energy disorder, to check the modeled two decay pathways of the mixed B800-B850 states (downward relaxation to $\mathrm{B} 800$ levels that are mainly " $\mathrm{B} 800$ " in character and to levels that are mainly "B850" in character). 


\section{APPENDIX. 2-MODE/3-GAUSSIAN HOLE PROFILE SIMULATION PROGRAM}

Following is the program used to test to fit the experimental hole spectra of FMO complex described in Chapter 3. The original program (1-Gaussian) was written by Dr. John Hayes in $\mathrm{C} / \mathrm{C}++$ to calculate hole profiles analytically in the short burn time limit, according to our groups' hole burning theory $[1,2]$. Variety of hole profile simulation programs have been written and revised by former members of our group, including Dr. Inja-Lee, Dr. Luchuan Shu, Dr. Paul Lyle and by Dr. John Hayes $[2,3]$.

My contribution to the program was the conversion of the program into Wolframs' Mathematica ${ }^{\circledR}$ script language (see Refs. [4-6] for details on formats and functions), which allows user to use actual integration for the absorption spectrum calculation rather than using approximation by Bode function. The program below (in cell-expression format) was written in compatible with Mathematica 2.2x and 3.0 and it was tested under 32bits Microsoft Windows version of 'Mathematica for Students version 3.0'. Descriptions of calculation variables and other information are included in the program itself. Combining the 2-mode profiles generated the 5-mode single-site absorption profile presented in Chapter 1.

One of the possible future works would be the incorporation of tunneling parameter (Gaussian distribution) into the hole-burning quantum yield variable, $\phi$, as discussed in recently published papers $[7,8]$. Also, revisions and algorithm changes may be necessary for faster calculation. 


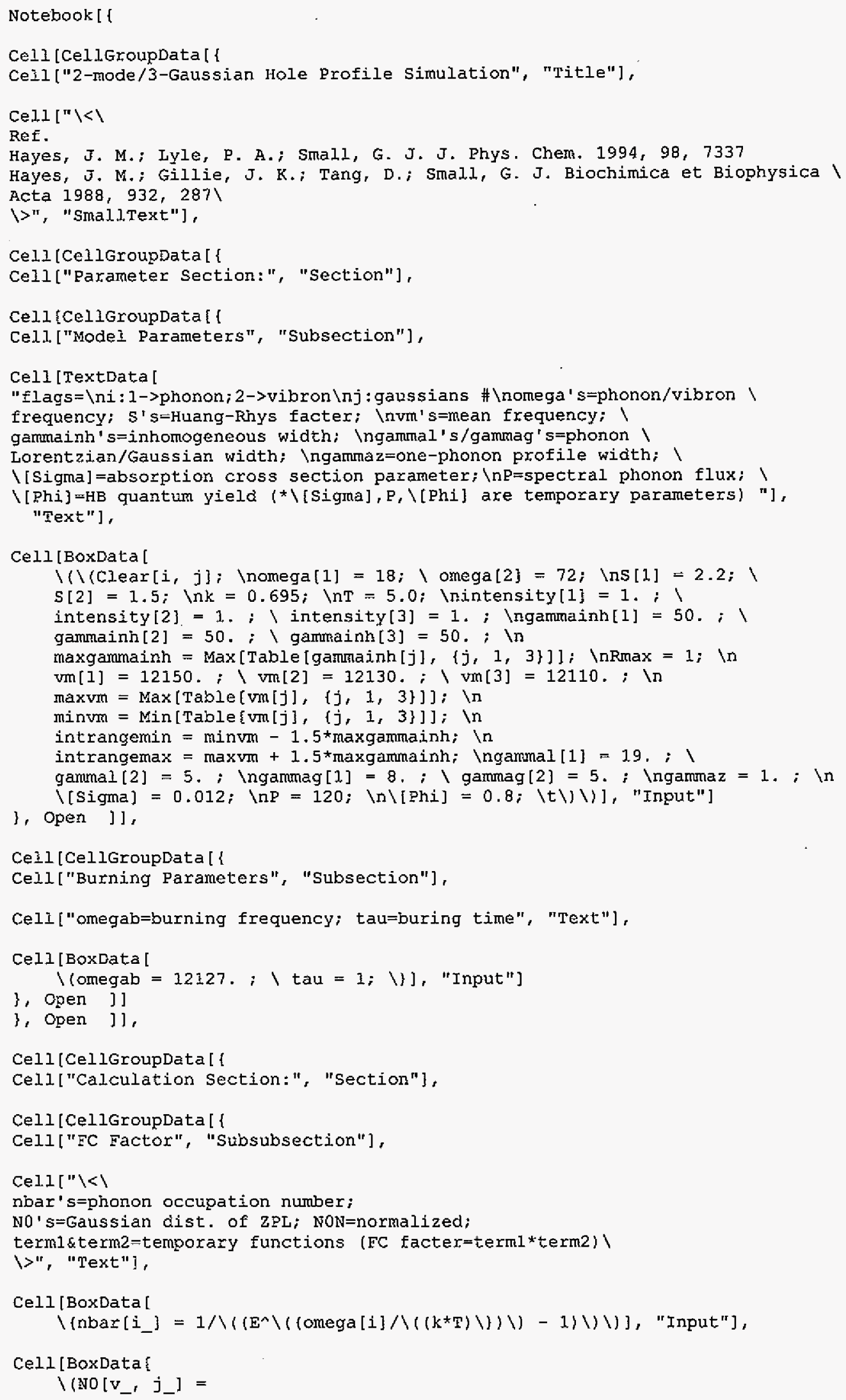




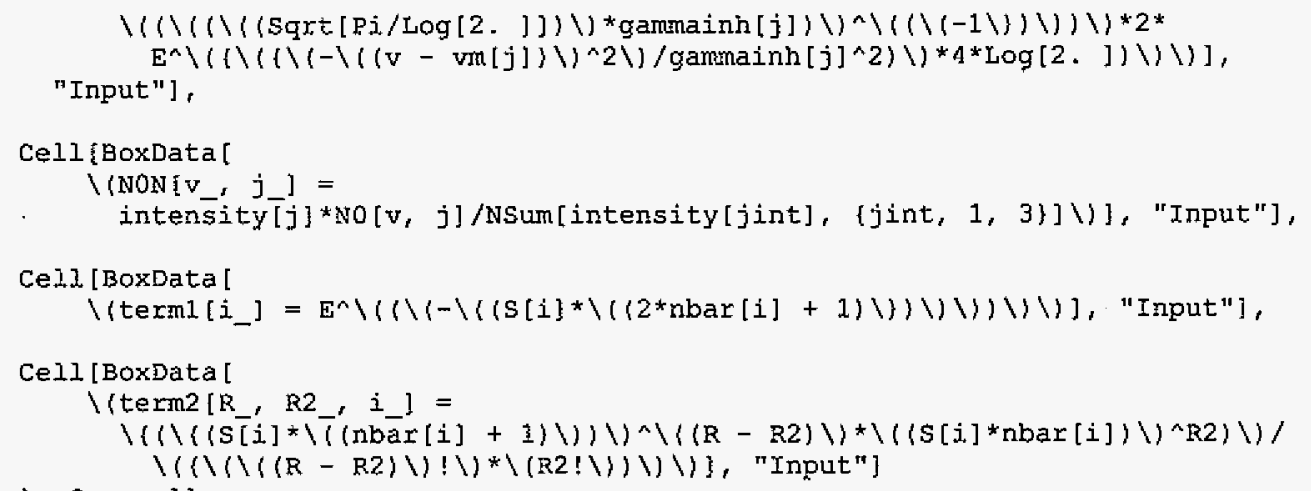




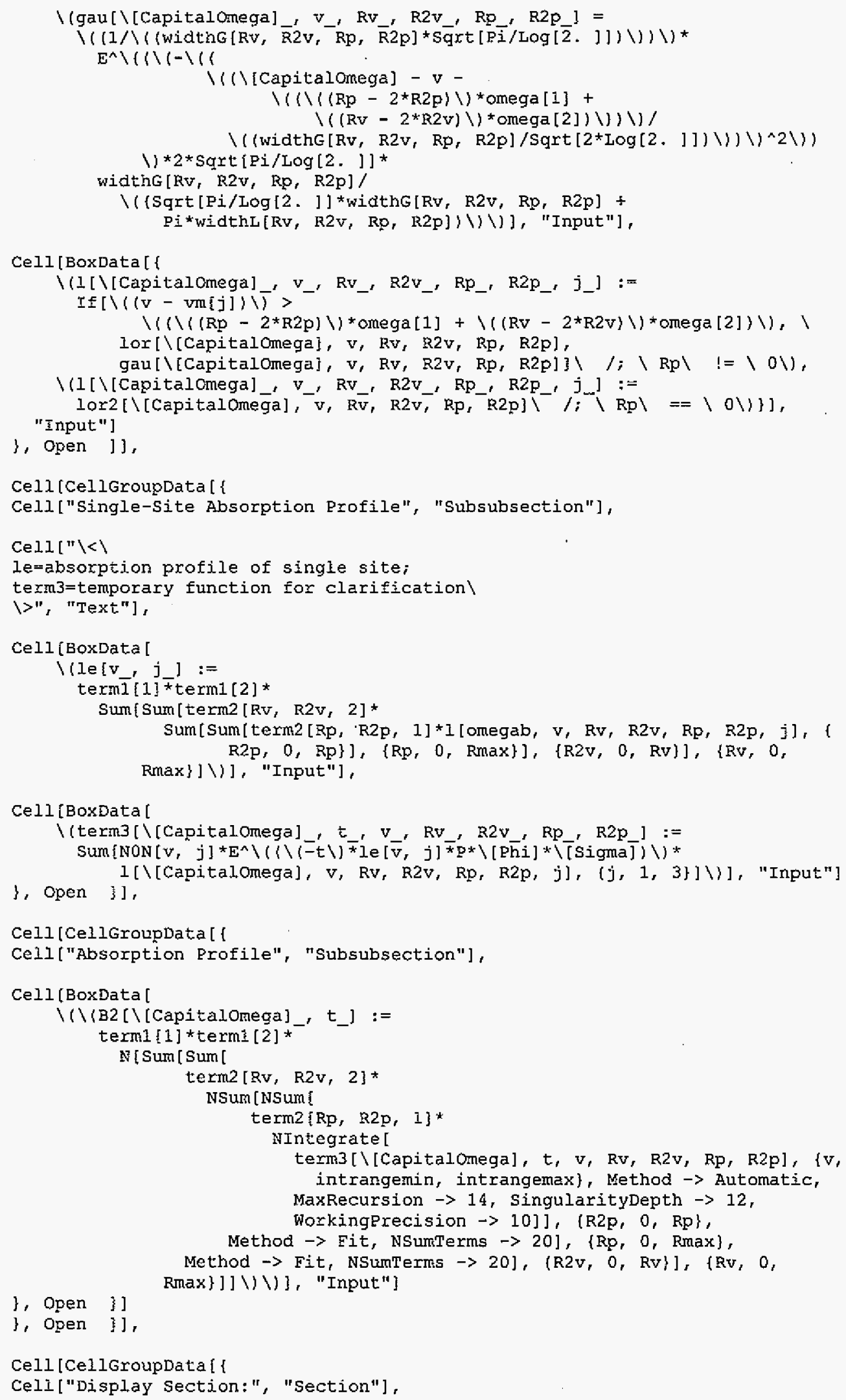




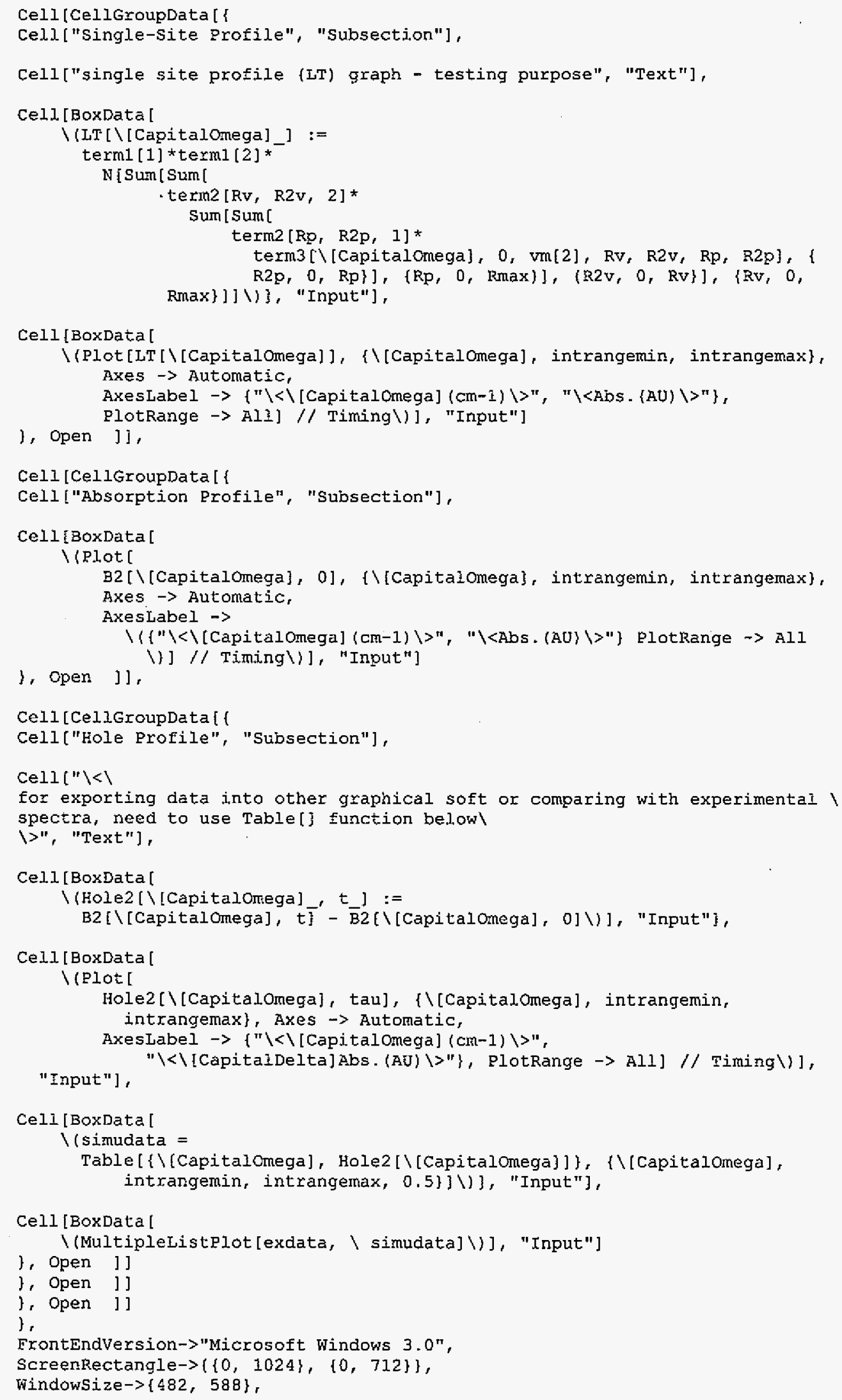


WindowMargins-> $\{\{4$, Automatic $\}$ \{Automatic, 5\}\},

PrintingCopies $>1$,

PrintingRageRange $\rightarrow$ Automatic, Automatic $\}$

]

\section{References:}

1. Hayes, J. M., Gillie, J. K., Tang, D. and Small, G. J. Biochimica et Biophysica Acta $1988,932,287$.

2. Hayes, J. M., Lyle, P. A. and Small, G. J. J. Phys. Chem. 1994, 98, 7337.

3. Lyle, P. A. Ph.D. Dissertation, Iowa State University, 1992.

4. Wolframs, S. Mathematica (second ed), 1991, Addison-Wesley, Reading, Massachusetts, pp.684-687.

5. Maeder, R. Programming in Mathematica (second ed.), 1991, Addison-Wesley, Reading, Massachusetts, p.69 and references therein.

6. Blachman, N. Mathematica: A Practical Approach, 1992, Prentice Hall, Engelwood Cliffs, New Jersey, pp. 36-42.

7. Reinot, T. and Small, G. J.J. Chem. Phys. 2000, 113, 10207.

8. Reinot, T. and Small, G. J. J. Chem. Phys. 2001, 114, 9105. 


\section{ACKNOWLEDGMENTS}

I would like to take this opportunity to express my genuine thanks to those who assisted me during of my thesis research.

I am indebted to my research advisor, Professor Gerald Small, for his guidance, patience and numerous discussions in the past few years. I am fortunate to benefit from his knowledge and insights in various subjects.

Dr. Valter Zazubovich and Dr. Margus Rätsep deserve my sincere appreciation and much credit for the work presented in this thesis. I would like to acknowledge Dr. John Hayes for providing invaluable advices. I am also particularly grateful to Dr. Tõnu Reinot for his various suggestions to this thesis work and providing me some great figures. I am also grateful to Dr. Ryszard Jankowiak for his assistance and expertise.

I am particularly thankful to Dr. Kenneth Roberts for helpful discussions and (soon to be Dr.) Richard Walsh who spent hours proofreading my writing.

Most of all, my sincere thank goes to Ms. Tiffany Fraser for her continuous support and understanding that kept me focused on completing my thesis studies.

This work was performed at Ames Laboratory under Contract No. W-7405-Eng-82 with the U.S. Department of Energy. The United States government has assigned the DOE Report number IS-T 2110 to this thesis. 